\title{
One-loop corrections to the two-body decays of the neutral Higgs bosons in the complex NMSSM
}

\author{
Julien Baglio ${ }^{1,2, a}$, Thi Nhung Dao ${ }^{3, b}$, Margarete Mühlleitner ${ }^{4, c}$ \\ ${ }^{1}$ Institute for Theoretical Physics, Eberhard Karls University Tübingen, Auf der Morgenstelle 14, 72076 Tübingen, Germany \\ 2 Theoretical Physics Department, CERN, 1211 Geneva 23, Switzerland \\ ${ }^{3}$ Institute For Interdisciplinary Research in Science and Education, ICISE, Khu Vuc 2, Ghenh Rang, Quy Nhon, Binh Dinh, Vietnam \\ ${ }^{4}$ Institute for Theoretical Physics, Karlsruhe Institute of Technology, Wolfgang-Gaede-Str. 1, 76131 Karlsruhe, Germany
}

Received: 8 July 2020 / Accepted: 3 October 2020 / Published online: 19 October 2020

(C) The Author(s) 2020

\begin{abstract}
Since no direct signs of new physics have been observed so far indirect searches in the Higgs sector have become increasingly important. With the discovered Higgs boson behaving very standard model (SM)-like, however, indirect new physics manifestations are in general expected to be small. On the theory side, this makes precision predictions for the Higgs parameters and observables indispensable. In this paper, we provide in the framework of the CP-violating next-to-minimal supersymmetric extension of the SM (NMSSM) the complete next-to-leading order (SUSY-)electroweak corrections to the neutral Higgs boson decays that are on-shell and non-loop induced. We also investigate possible gauge-dependence issues in the decays of light Higgs bosons into fermion pairs. Together with the also provided SUSY-QCD corrections to colored final states, the newly calculated SUSY-electroweak corrections are implemented in the Fortran code NMSSMCALC which already includes the state-of-the art QCD corrections. The new code is called NMSSMCALCEW. This way we provide the NMSSM Higgs boson decays and branching ratios at presently highest possible precision and thereby contribute to the endeavor of searching for New Physics at present and future colliders.
\end{abstract}

\section{Introduction}

The discovery of a scalar particle by the LHC experiments ATLAS [1] and CMS [2] and the subsequent investigation of its properties revealed a Higgs boson that behaves very standard model (SM)-like. Also years after its discovery there are no evidences for new physics from direct searches. In

\footnotetext{
a e-mail: julien.baglio@cern.ch

b e-mail: dtnhung@ifirse.icise.vn (corresponding author)

c e-mail: margarete.muehlleitner@kit.edu
}

this situation the precise investigation of the Higgs sector plays an important role. Indirect effects of physics beyond the SM (BSM) might show up in the properties of the discovered Higgs boson. With a mass of $125.09 \mathrm{GeV}$ [3] it does not exclude the possibility for the Higgs boson of a supersymmetric (SUSY) extension of the SM, like the minimal (MSSM) or the next-to-minimal (NMSSM) ones. Supersymmetry certainly belongs to the best motivated and most intensively studied BSM extensions, and the NMSSM, with a Higgs sector consisting of seven Higgs bosons arising after electroweak symmetry breaking (EWSB) from the two doublet and singlet fields of the Higgs sector, provides a rich phenomenology $[4,5]$. The experimental limits strongly restrict possible new physics effects in the Higgs sector and call for precision in the theory predictions for the Higgs boson observables. This is also necessary in order to be able to distinguish between new physics extensions in case of discovery.

In this paper, we concentrate on the NMSSM Higgs boson decays. While the (SUSY-)QCD corrections can be taken over from the MSSM case with the appropriate modifications and a minimum of effort, this is not the case for the electroweak (EW) corrections. In the recent years there has been some progress on this subject. In the CP-conserving NMSSM, members of our group computed the next-toleading order (NLO) SUSY-EW and SUSY-QCD corrections to the decays of CP-odd NMSSM Higgs bosons into stop pairs and found that both the EW and the SUSY-QCD corrections are significant and can be of opposite sign [6]. The authors of $[7,8]$ provided in the framework of the CPconserving NMSSM its full one-loop renormalization and the two-body Higgs decays at one-loop order in the on-shell (OS) renormalization scheme. A generic calculation of the twobody partial decays widths at full one-loop level was provided in [9] in the $\overline{\mathrm{DR}}$ scheme. In [10] the full one-loop corrections for the neutral CP-violating NMSSM Higgs bosons were 
calculated to their decays into fermions and gauge bosons and combined with the leading QCD corrections. For the Higgs-to-Higgs decays, we provided in previous papers the complete one-loop [11] and the order $\mathcal{O}\left(\alpha_{t} \alpha_{s}\right)$ two-loop [12] corrections in the $\mathrm{CP}$-conserving and CP-violating NMSSM, respectively.

In this work, we compute, in the framework of the CP-violating NMSSM, the complete next-to-leading order (SUSY-)electroweak corrections to the neutral NMSSM Higgs boson decays into all tree-level induced SM final states, i.e. into fermion and massive gauge boson pairs, but also into non-SM pairs, namely gauge and Higgs boson final states, chargino and neutralino pairs, and into squarks. ${ }^{1}$ Where applicable we combine our corrections with the already available (SUSY-)QCD corrections. We furthermore include the complete one-loop corrections to the decays into Higgs boson pairs, $c f$. Refs. [11,12]. We also include a discussion of the gauge dependence of the computed decay widths. We explicitly show that it does not arise from the pure electroweak one-loop corrections but is due to the mixing of different higher orders that arises from the inclusion of higher loop-corrected Higgs boson masses and resummed wave function renormalization factors $\mathbf{Z}^{H}$. This issue has been well known and needs further detailed investigations that are beyond the scope of this paper. We note that our nominal predictions for the loop-corrected Higgs masses and for the loop-corrected decay widths have been calculated in the 'tHooft-Feynman gauge (i.e. the gauge-fixing parameters have been set to one). For the loop-induced decays into gluon and photon pairs as well as a photon and a $Z$ boson no corrections are provided as they would be of twoloop order. For the first time, we present the one-loop corrections to the electroweakino, stop and sbottom masses in the context of the CP-violating NMSSM, by applying both $\mathrm{OS}$ and $\overline{\mathrm{DR}}$ schemes. We have implemented our corrections in our original code NMSSMCALC [14], which calculates, based on a mixed OS- $\overline{\mathrm{DR}}$ scheme, the NMSSM Higgs mass corrections and decays in both the CP-conserving and CPviolating case. This way we provide the NMSSM Higgs boson decays and branching ratios at presently highest possible precision including the state-of-the-art (SUSY-)QCD and the computed (SUSY-)EW corrections. In the EW higherorder corrections we not only include the NLO vertex corrections but also take into account the proper on-shell conditions of the external decaying Higgs bosons up to twoloop order $\mathcal{O}\left(\alpha_{t} \alpha_{s}+\alpha_{t}^{2}\right)$. This is the order up to which the mass corrections for the NMSSM Higgs bosons both

\footnotetext{
${ }^{1}$ In Ref. [13], the impact of the EW corrections to heavy NMSSM Higgs boson decays into fermion pairs was discussed. It was shown that these contributions are dominated by Sudakov double logarithms. The authors furthermore stressed the relevance of three-body decays for a consistent evaluation of the total widths and branching ratios at complete one-loop order.
}

in the CP-conserving [15] and CP-violating case [16-18] have been implemented in NMSSMCALC. The new program is called NMSSMCALCEW and can be obtained at the url: https:// www.itp.kit.edu/ maggie/NMSSMCALCEW/. Here also a detailed description of the program and its structure are given, instructions on how to compile and run it as well as information on modifications, which is constantly updated. A brief description of the code is given in "Appendix B".

The paper is organized as follows. In Sect. 2 we introduce the NMSSM sectors at tree level, that are relevant for our computation, and set our notation before we move on to the NMSSM at one-loop level in Sect. 3. We here describe the renormalization of the Higgs, chargino/neutralino, and squark sectors as well as the loop corrections to the Higgs boson masses and mixings, to the neutralino and chargino masses, and finally to the squark masses and their mixings. Section 4 is devoted to the detailed presentation of our calculation of the one-loop corrections to the neutral non-loop induced Higgs boson decays into on-shell final states, namely into fermion pairs, massive gauge boson pairs, final states with one gauge and one Higgs boson, neutralino and chargino pairs, and squark final states. In Sect. 5 we present the numerical analysis of the one-loop corrections to the Higgs boson branching ratios into SM and SUSY final states, where we discuss in particular the size of the newly implemented corrections to both the branching ratios and to the electroweakino and third generation squark masses. A discussion on the gauge-parameter dependence issue in the Higgs boson decays into fermion pairs is presented in Sect. 5.6. Our conclusions are given in Sect. 6. Explicit expressions of the counterterm couplings for the decays of neutral Higgs bosons into a squark pair are displayed in "Appendix A".

\section{The NMSSM at tree level}

We are working in the complex NMSSM with a preserved $\mathbb{Z}_{3}$ symmetry. The Lagrangian of the NMSSM can be divided into the Lagrangian of the MSSM without $\mathbb{Z}_{3}$-symmetryviolating terms such as $\mu$ - and $B_{\mu}$-terms and the additional part coming from the NMSSM. For convenience of the reader and to set our notation, we give here the parts of the Lagrangian that are relevant for our calculations. For the Higgs sector we need the NMSSM Higgs potential. It is derived from the NMSSM superpotential $W_{\mathrm{NMSSM}}$, the corresponding soft SUSY-breaking terms, and the $D$-term contributions. With the Higgs doublet superfields $\hat{H}_{u}$ and $\hat{H}_{d}$ coupling to the up- and down-type quark superfields, respectively, and the singlet superfield $\hat{S}$, we have for the NMSSM superpotential

$$
W_{\mathrm{NMSSM}}=W_{\mathrm{MSSM}}-\epsilon_{a b} \lambda \hat{S} \hat{H}_{d}^{a} \hat{H}_{u}^{b}+\frac{1}{3} \kappa \hat{S}^{3}
$$


where $a, b=1,2$ are the indices of the fundamental $S U(2)_{L}$ representation and $\epsilon_{a b}$ is the totally antisymmetric tensor with $\epsilon_{12}=\epsilon^{12}=1$. The MSSM part reads

$$
\begin{aligned}
W_{\mathrm{MSSM}}= & -\epsilon_{a b}\left(y_{u} \hat{H}_{u}^{a} \hat{Q}^{b} \hat{U}^{c}\right. \\
& \left.-y_{d} \hat{H}_{d}^{a} \hat{Q}^{b} \hat{D}^{c}-y_{e} \hat{H}_{d}^{a} \hat{L}^{b} \hat{E}^{c}\right),
\end{aligned}
$$

in terms of the left-handed quark and lepton superfield doublets $\hat{Q}$ and $\hat{L}$ and the right-handed up-type, down-type, and electron-type superfield singlets $\hat{U}, \hat{D}$, and $\hat{E}$, respectively. Charge conjugation is denoted by the superscript $c$, and color and generation indices have been omitted. The NMSSM superpotential contains the coupling $\kappa$ of the selfinteraction of the new singlet superfield and the coupling $\lambda$ for the $\hat{S}$ interaction with the two Higgs doublet superfields. Both couplings are complex. The quark and lepton Yukawa couplings $y_{d}, y_{u}$, and $y_{e}$ are in general complex. However, in case of no generation mixing, as assumed in this paper, the phases of the Yukawa couplings can be absorbed through a redefinition of the quark fields, so that the phases can be chosen arbitrarily without changing the physical meaning [19]. The soft SUSY-breaking NMSSM Lagrangian in terms of the component fields $H_{u}, H_{d}$ and $S$ reads

$$
\begin{aligned}
\mathcal{L}_{\mathrm{NMSSM}}^{\mathrm{soft}}= & \mathcal{L}_{\mathrm{MSSM}}^{\mathrm{soft}}-m_{S}^{2}|S|^{2} \\
& +\left(\epsilon_{a b} A_{\lambda} \lambda S H_{d}^{a} H_{u}^{b}-\frac{1}{3} A_{\kappa} \kappa S^{3}+\text { h.c. }\right) .
\end{aligned}
$$

It contains two more complex parameters specific to the NMSSM, the soft SUSY-breaking trilinear couplings $A_{\lambda}$ and $A_{\kappa}$. The soft SUSY-breaking MSSM contribution can be cast into the form

$$
\begin{aligned}
\mathcal{L}_{\mathrm{MSSM}}^{\mathrm{soft}}= & -m_{H_{d}}^{2}\left|H_{d}\right|^{2}-m_{H_{u}}^{2}\left|H_{u}\right|^{2}-m_{\tilde{Q}^{2}}^{2}|\tilde{Q}|^{2}-m_{\tilde{u}_{R}}^{2}\left|\tilde{u}_{R}\right|^{2} \\
& -m_{\tilde{d}_{R}}^{2}\left|\tilde{d}_{R}\right|^{2}-\left.m_{\tilde{L}}^{2} \tilde{L}\right|^{2}-m_{\tilde{e}_{R}}^{2}\left|\tilde{e}_{R}\right|^{2} \\
& +\epsilon_{a b}\left(y_{u} A_{u} H_{u}^{a} \tilde{Q}^{b} \tilde{u}_{R}^{*}-y_{d} A_{d} H_{d}^{a} \tilde{Q}^{b} \tilde{d}_{R}^{*}\right. \\
& \left.-y_{e} A_{e} H_{d}^{a} \tilde{Q}^{b} \tilde{e}_{R}^{*}+\text { h.c. }\right) \\
& -\frac{1}{2}\left(M_{1} \tilde{B} \tilde{B}+M_{2} \tilde{W}_{i} \tilde{W}_{i}+M_{3} \tilde{G} \tilde{G}+h . c\right)
\end{aligned}
$$

where the SM-type and SUSY fields corresponding to a superfield (denoted with a hat) are represented by a letter without and with a tilde, respectively. The indices $\tilde{Q}$ $(\tilde{L})$ of the soft SUSY-breaking masses denote, exemplary for the first generation, the left-handed quark (lepton) doublet component fields of the corresponding quark and lepton superfields, and $\tilde{u}_{R}, \tilde{d}_{R}, \tilde{e}_{R}$ the right-handed component fields for the up-type and down-type quarks, and charged leptons, respectively. The trilinear couplings $A_{u}, A_{d}$ and $A_{e}$ of the up-type and down-type quarks and charged leptons are in general complex, whereas the soft SUSY-breaking mass terms $m_{x}^{2}\left(x=S, H_{u}, H_{d}, \tilde{Q}, \tilde{u}_{R}, \tilde{d}_{R}, \tilde{L}, \tilde{e}_{R}\right)$ are real. The soft SUSY-breaking mass parameters of the gauginos, $M_{1}$, $M_{2}, M_{3}$, for the bino, the winos, and the gluinos, $\tilde{B}, \tilde{W}_{i}$ $(i=1,2,3)$, and $\tilde{G}$, corresponding to the weak hypercharge $U(1)$, the weak isospin $S U(2)$, and the color $S U(3)$ symmetry, are in general complex. The $R$-symmetry can be exploited to choose either $M_{1}$ or $M_{2}$ to be real. In this paper we keep both $M_{1}$ and $M_{2}$ complex.

Expanding the scalar Higgs fields about their vacuum expectation values (VEVs) $v_{u}, v_{d}$, and $v_{s}$, two further phases, $\varphi_{u}$ and $\varphi_{s}$, are introduced which describe the phase differences between the VEVs,

$$
\begin{aligned}
H_{d} & =\left(\begin{array}{c}
\frac{1}{\sqrt{2}}\left(v_{d}+h_{d}+i a_{d}\right) \\
h_{d}^{-}
\end{array}\right), \\
H_{u} & =e^{i \varphi_{u}}\left(\begin{array}{c}
h_{u}^{+} \\
\frac{1}{\sqrt{2}}\left(v_{u}+h_{u}+i a_{u}\right)
\end{array}\right), \\
S & =\frac{e^{i \varphi_{s}}}{\sqrt{2}}\left(v_{s}+h_{s}+i a_{s}\right) .
\end{aligned}
$$

For vanishing phases, the fields $h_{i}$ and $a_{i}$ with $i=d, u, s$ correspond to the $\mathrm{CP}$-even and $\mathrm{CP}$-odd part of the neutral entries of $H_{d}, H_{u}$ and $S$. The charged components are denoted by $h_{d, u}^{ \pm}$. In this paper, we set the phases of the Yukawa couplings to zero. We furthermore re-phase the left- and right-handed up-quark fields as $u_{L} \rightarrow e^{-i \varphi_{u}} u_{L}$ and $u_{R} \rightarrow e^{i \varphi_{u}} u_{R}$, so that the quark and lepton mass terms yield real masses.

After electroweak symmetry breaking (EWSB) the six Higgs interaction states mix and in the basis $\phi=\left(h_{d}, h_{u}, h_{s}\right.$, $\left.a_{d}, a_{u}, a_{s}\right)^{T}$ the mass term is given by

$\mathcal{L}_{\text {neutral }}^{m}=\frac{1}{2} \phi^{T} M_{\phi \phi} \phi$.

The mass matrix $M_{\phi \phi}$ is obtained from the second derivative of the Higgs potential with respect to the Higgs fields in the vacuum. The explicit expression of the mass matrix $M_{\phi \phi}$ can be found in Ref. [16]. The transformation into mass eigenstates at tree level can be performed with orthogonal matrices $\mathcal{R}, \mathcal{R}^{G}$,

$$
\begin{aligned}
\operatorname{diag}\left(m_{h_{1}}^{2}, m_{h_{2}}^{2}, m_{h_{3}}^{2}, m_{h_{4}}^{2}, m_{h_{5}}^{2}, M_{Z}^{2}\right) \\
=\mathcal{R R}^{G} M_{\phi \phi}\left(\mathcal{R} \mathcal{R}^{G}\right)^{T} \\
\left(h_{1}, h_{2}, h_{3}, h_{4}, h_{5}, G\right)^{T}=\mathcal{R} \mathcal{R}^{G}\left(h_{d}, h_{u}, h_{s}, a_{d}, a_{u}, a_{s}\right)^{T} \\
=\mathcal{R}\left(h_{d}, h_{u}, h_{s}, a, a_{s}, G\right)^{T},
\end{aligned}
$$

where the matrix $\mathcal{R}^{G}$ is used first to single out the Goldstone boson $G$ whose mass is equal to the $Z$-boson mass, $M_{Z}$, in the 'tHooft-Feynman gauge. The tree-level Higgs mass 
eigenstates are denoted by the small letter $h$, and their masses are ordered as $m_{h_{1}} \leq \cdots \leq m_{h_{5}}$.

The mass term for the charged components of the Higgs doublets in

$\mathcal{L}_{\text {charged }}^{m}=\left(h_{d}^{+}, h_{u}^{+}\right) M_{h^{+} h^{+}}\left(\begin{array}{l}h_{d}^{-} \\ h_{u}^{-}\end{array}\right)$,

in general $R_{\xi}$ gauge, is given by

$$
\begin{aligned}
M_{h^{+} h^{+}}= & \frac{1}{2}\left(\begin{array}{cc}
t_{\beta} & 1 \\
1 & 1 / t_{\beta}
\end{array}\right) \\
& \times\left[M_{W}^{2} s_{2 \beta}+\frac{|\lambda| v_{s}}{\cos \left(\varphi_{\lambda}+\varphi_{u}+\varphi_{s}\right)}\right. \\
& \times\left(\sqrt{2} \operatorname{Re} A_{\lambda}+|\kappa| v_{s} \cos \left(\varphi_{\kappa}+3 \varphi_{s}\right)\right) \\
& \left.-\frac{2|\lambda|^{2} M_{W}^{2} s_{\theta_{W}}^{2}}{e^{2}} s_{2 \beta}\right] \\
& +M_{W}^{2} \xi_{W}\left(\begin{array}{cc}
c_{\beta}{ }^{2} & -c_{\beta} s_{\beta} \\
-c_{\beta} s_{\beta} & s_{\beta}^{2}
\end{array}\right),
\end{aligned}
$$

where $M_{W}$ is the mass of the $W$ boson, $\xi_{W}$ is the $W$-boson gauge parameter, $\theta_{W}$ the electroweak mixing angle, $e$ the electric charge and $\varphi_{\lambda}, \varphi_{\kappa}$ the complex phases of $\lambda$ and $\kappa$, respectively. The angle $\beta$ is defined as

$\tan \beta=v_{u} / v_{d}$.

Here and in the following we use the short hand notation $c_{x}=\cos x, s_{x}=\sin x$ and $t_{x}=\tan x$. Diagonalizing this mass matrix by a rotation matrix with the angle $\beta_{c}$, for which at Born level $\beta_{c}=\beta$, one obtains the charged Higgs boson mass as

$$
\begin{aligned}
& M_{H^{ \pm}}^{2}=M_{W}^{2}+\frac{|\lambda| v_{s}}{s_{2 \beta} \cos \left(\varphi_{\lambda}+\varphi_{u}+\varphi_{s}\right)} \\
& \quad \times\left(\sqrt{2} \operatorname{Re} A_{\lambda}+|\kappa| v_{s} \cos \left(\varphi_{\kappa}+3 \varphi_{s}\right)\right)-\frac{2|\lambda|^{2} M_{W}^{2} s_{\theta_{W}}^{2}}{e^{2}} .
\end{aligned}
$$

The mass of the charged Goldstone boson $G^{ \pm}$is $\sqrt{\xi_{W}} M_{W}$. We work in the 't Hooft-Feynman gauge $\left(\xi_{W}=1\right)$, therefore its mass is equal to $M_{W}$.

The fermionic superpartners of the neutral Higgs bosons, $\tilde{H}_{d}^{0}, \tilde{H}_{u}^{0}, \tilde{S}$, and of the neutral gauge bosons, $\tilde{B}, \tilde{W}_{3}$, mix, and in the Weyl spinor basis $\psi^{0}=\left(\tilde{B}, \tilde{W}_{3}, \tilde{H}_{d}^{0}, \tilde{H}_{u}^{0}, \tilde{S}\right)^{T}$ the neutralino mass matrix $M_{N}$ is given by after EWSB. The symmetric neutralino mass matrix can be diagonalized by a $5 \times 5$ matrix $N$, yielding $\operatorname{diag}\left(m_{\tilde{\chi}_{1}^{0}}\right.$, $\left.m_{\tilde{\chi}_{2}^{0}}, m_{\tilde{\chi}_{3}^{0}}, m_{\tilde{\chi}_{4}^{0}}, m_{\tilde{\chi}_{5}^{0}}\right)=N^{*} M_{N} N^{\dagger}$. In the diagonalization process, the $i^{\text {th }}$ mass eigenvalue $m_{i}$ can have an arbitrary complex phase, we choose to make it positive by applying $N_{i j} \rightarrow N_{i j} \sqrt{m_{i} /\left|m_{i}\right|}, j=1, \ldots, 5$. As a result, the neutralino masses are positive and are ordered as $m_{\tilde{\chi}_{1}^{0}} \leq \cdots \leq$ $m_{\tilde{\chi}_{5}^{0}}$. The neutralino mass eigenstates $\tilde{\chi}_{i}^{0}$, expressed as Majorana spinors, can then be obtained by

$\tilde{\chi}_{i}^{0}=\left(\frac{\chi_{i}^{0}}{\chi_{i}^{0}}\right) \quad$ with $\quad \chi_{i}^{0}=N_{i j} \psi_{j}^{0}, \quad i, j=1, \ldots, 5$,

where, in terms of the Pauli matrix $\sigma_{2}$,

$\overline{\chi_{i}^{0}}=i \sigma_{2} \chi_{i}^{0 *}$.

The fermionic superpartners of the charged Higgs and gauge bosons are given in terms of the Weyl spinors $\tilde{H}_{d}^{ \pm}, \tilde{H}_{u}^{ \pm}, \tilde{W}^{-}$ and $\tilde{W}^{+}$. With

$\psi_{R}^{-}=\left(\begin{array}{c}\tilde{W}^{-} \\ \tilde{H}_{d}^{-}\end{array}\right) \quad$ and $\quad \psi_{L}^{+}=\left(\begin{array}{c}\tilde{W}^{+} \\ \tilde{H}_{u}^{+}\end{array}\right)$

the mass term for these spinors is of the form

$\mathcal{L}=\left(\psi_{R}^{-}\right)^{T} M_{C} \psi_{L}^{+}+$h.c.,

where

$M_{C}=\left(\begin{array}{cc}M_{2} & \sqrt{2} s_{\beta} M_{W} e^{-i \varphi_{u}} \\ \sqrt{2} c_{\beta} M_{W} & \lambda \frac{v_{s}}{\sqrt{2}} e^{i \varphi_{s}}\end{array}\right)$

The chargino mass matrix $M_{C}$ can be diagonalized with the help of two unitary $2 \times 2$ matrices, $U$ and $V$, yielding

$\operatorname{diag}\left(m_{\tilde{\chi}_{1}^{ \pm}}, m_{\tilde{\chi}_{2}^{ \pm}}\right)=U^{*} M_{C} V^{\dagger}$,

with $m_{\tilde{\chi}_{1}^{ \pm}} \leq m_{\tilde{\chi}_{2}^{ \pm}}$. The left-handed and the right-handed part of the mass eigenstates are

$\tilde{\chi}_{L}^{+}=V \psi_{L}^{+} \quad$ and $\quad \tilde{\chi}_{R}^{-}=U \psi_{R}^{-}$,

respectively, with the mass eigenstates $(i=1,2)$

$$
M_{N}=\left(\begin{array}{ccccc}
M_{1} & 0 & -c_{\beta} M_{Z} s_{\theta_{W}} & M_{Z} s_{\beta} s_{\theta_{W}} e^{-i \varphi_{u}} & 0 \\
0 & M_{2} & c_{\beta} M_{W} & -M_{W} s_{\beta} e^{-i \varphi_{u}} & 0 \\
-c_{\beta} M_{Z} s_{\theta_{W}} & c_{\beta} M_{W} & 0 & -\lambda \frac{v_{s}}{\sqrt{2}} e^{i \varphi_{s}} & -\frac{\sqrt{2} M_{W} s_{\beta} s_{\theta_{W}} \lambda e^{i \varphi_{u}}}{e} \\
M_{Z} s_{\beta} s_{\theta_{W}} e^{-i \varphi_{u}} & -M_{W} s_{\beta} e^{-i \varphi_{u}} & -\lambda \frac{v_{s}}{\sqrt{2}} e^{i \varphi_{s}} & 0 & -\frac{\sqrt{2} M_{W} c_{\beta} s_{\theta_{W}} \lambda}{e} \\
0 & 0 & -\frac{\sqrt{2} M_{W} s_{\beta} s_{\theta_{W}} \lambda e^{i \varphi_{u}}}{e} & -\frac{\sqrt{2} M_{W} c_{\beta} s_{\theta_{W}} \lambda}{e} & \sqrt{2} \kappa v_{s} e^{i \varphi_{s}}
\end{array}\right)
$$


$\tilde{\chi}_{i}^{+}=\left(\frac{\tilde{\chi}_{L_{i}}^{+}}{\tilde{\chi}_{R_{i}}^{-}}\right)$

written as Dirac spinors. In summary, the bilinear terms in the chargino and neutralino mass eigenstates are given by

$$
\begin{aligned}
\mathcal{L}= & \overline{\tilde{\chi}_{i}^{+}} p P_{L} \tilde{\chi}_{i}^{+}+\overline{\tilde{\chi}_{i}^{+}} p P_{R} \tilde{\chi}_{i}^{+}-\overline{\tilde{\chi}_{i}^{+}}\left[U^{*} M_{C} V^{\dagger}\right]_{i j} P_{L} \tilde{\chi}_{j}^{+} \\
& -\overline{\tilde{\chi}_{i}^{+}}\left[V M_{C}^{\dagger} U^{T}\right]_{i j} P_{R} \tilde{\chi}_{j}^{+} \\
& +\overline{\tilde{\chi}_{k}^{0}} p P_{L} \tilde{\chi}_{k}^{0}+\overline{\tilde{\chi}_{k}^{0}} p P_{R} \tilde{\chi}_{k}^{0}-\overline{\tilde{\chi}_{k}^{0}}\left[N^{*} M_{N} N^{\dagger}\right]_{k l} P_{L} \tilde{\chi}_{l}^{0} \\
& -\overline{\tilde{\chi}_{k}^{0}}\left[N M_{N}^{\dagger} N^{T}\right]_{k l} P_{R} \tilde{\chi}_{l}^{0},
\end{aligned}
$$

where the left- and right-handed projectors are defined as $P_{L / R}=\left(1 \mp \gamma_{5}\right) / 2$ and $i, j=1,2$ and $k, l=1, \ldots, 5$.

The scalar partners of the left- and right-handed quarks are denoted by $\tilde{q}_{L}$ and $\tilde{q}_{R}$, respectively. The mixing matrix for the top squark is given by

$M_{\tilde{t}}=\left(\begin{array}{cc}m_{\tilde{Q}_{3}}^{2}+m_{t}^{2}+M_{Z}^{2} c_{2 \beta}\left(\frac{1}{2}-\frac{2}{3} s_{\theta_{W}}^{2}\right) & m_{t}\left(A_{t}^{*} e^{-i \varphi_{u}}-\mu_{\mathrm{eff}} / t_{\beta}\right) \\ m_{t}\left(A_{t} e^{i \varphi_{u}}-\mu_{\mathrm{eff}}^{*} / t_{\beta}\right) & m_{t}^{2}+m_{\tilde{t}_{R}}^{2}+\frac{2}{3} M_{Z}^{2} c_{2 \beta} s_{\theta_{W}}^{2}\end{array}\right)$,

while the bottom squark matrix reads

$M_{\tilde{b}}=\left(\begin{array}{cc}m_{\tilde{Q}_{3}}^{2}+m_{b}^{2}+M_{Z}^{2} c_{2 \beta}\left(-\frac{1}{2}+\frac{1}{3} s_{\theta_{W}}^{2}\right) & m_{b}\left(A_{b}^{*}-e^{i \varphi_{u}} \mu_{\mathrm{eff}} t_{\beta}\right) \\ m_{b}\left(A_{b}-e^{-i \varphi_{u}} \mu_{\mathrm{eff}}^{*} t_{\beta}\right) & m_{b}^{2}+m_{\tilde{b}_{R}}^{2}-\frac{1}{3} M_{Z}^{2} c_{2 \beta} s_{\theta_{W}}^{2}\end{array}\right)$,

where

$\mu_{\mathrm{eff}}=\frac{\lambda v_{s} e^{i \varphi_{s}}}{\sqrt{2}}$.

The mass eigenstates are obtained by diagonalizing these squark matrices with the unitary transformations

$$
\begin{aligned}
\operatorname{diag}\left(m_{\tilde{q}_{1}}^{2}, m_{\tilde{q}_{2}}^{2}\right) & =U^{\tilde{q}} M_{\tilde{q}} U^{\tilde{q} \dagger}, \\
\left(\begin{array}{c}
\tilde{q}_{1} \\
\tilde{q}_{2}
\end{array}\right) & =U^{\tilde{q}}\left(\begin{array}{c}
\tilde{q}_{L} \\
\tilde{q}_{R}
\end{array}\right), \quad q=t, b,
\end{aligned}
$$

with the usual convention $m_{\tilde{q}_{1}} \leq m_{\tilde{q}_{2}}$.

\section{The NMSSM at one-loop level}

\subsection{Renormalization}

\subsubsection{The Higgs sector}

For the Higgs sector we follow the mixed OS- $\overline{\mathrm{DR}}$ renormalization scheme described and applied in Refs. [15-18]. We do not repeat all details here but quote the most important formulae. There are eighteen parameters entering the Higgs sector at tree level,

$$
\begin{aligned}
& m_{H_{d}}^{2}, m_{H_{u}}^{2}, m_{S}^{2}, M_{W}^{2}, M_{Z}^{2}, e, \tan \beta, v_{s}, \varphi_{s}, \\
& \quad \varphi_{u},|\lambda|, \varphi_{\lambda},|\kappa|, \varphi_{\kappa},
\end{aligned}
$$

$\operatorname{Re} A_{\lambda}, \operatorname{Im} A_{\lambda}, \operatorname{Re} A_{\kappa}, \operatorname{Im} A_{\kappa}$.

Note that for the sake of convenience we decompose the complex trilinear couplings $A_{\lambda}$ and $A_{\kappa}$ into a real and imaginary part in contrast to Ref. [16] where the absolute values and complex phases were used. It was found in Ref. [16] that the four complex phases $\varphi_{s}, \varphi_{u}, \varphi_{\lambda}$ and $\varphi_{\kappa}$ do not need to be renormalized at the one-loop level, which can be inferred from the existing renormalization group equations (RGEs). We verified this statement and will discard them in our renormalization procedure.

In our introduction of the NMSSM Higgs sector in Sect. 2 we have already replaced the $U(1)$ and $S U(2)$ gauge couplings $g^{\prime}$ and $g$ and the VEV $v$ by the physical observables $M_{W}, M_{Z}$ and $e$. It is convenient to further convert, where possible, the input parameters in Eq. (3.1) to parameters that can be interpreted more easily in terms of physical quantities. Thus we trade the three soft SUSY-breaking mass parameters $m_{H_{d}}^{2}, m_{H_{u}}^{2}, m_{S}^{2}$ as well as $\operatorname{Im} A_{\lambda}$ and $\operatorname{Im} A_{\kappa}$ for the tadpole parameters $t_{\phi}\left(\phi=h_{d}, h_{u}, h_{s}, a_{d}, a_{s}\right)$. These coefficients of the terms of the Higgs potential $V_{\text {Higgs }}$ are linear in the Higgs boson fields and have to vanish, in order to ensure the minimum at non-vanishing VEVs $v_{u}, v_{d}, v_{s}$,

$\left.t_{\phi} \equiv \frac{\partial V_{\text {Higgs }}}{\partial \phi}\right|_{\text {Min. }} \stackrel{!}{=} 0$.

It is debatable whether the tadpole parameters can be called physical quantities, but certainly their introduction is motivated by physical interpretation. In the same way, in a slight abuse of the language, we will call the renormalization conditions for the tadpole parameters on-shell. With the new set of input parameters, we allow for two possible renormalization schemes in our Higgs mass calculation. The difference between the two schemes relates to the treatment of the charged Higgs mass. In the first scheme the charged Higgs mass is an OS input parameter,

$\underbrace{t_{h_{d}}, t_{h_{u}}, t_{h_{s}}, t_{a_{d}}, t_{a_{s}}, M_{H^{ \pm}}^{2}, M_{W}^{2}, M_{Z}^{2}, e}_{\text {on-shell }}, \underbrace{\tan \beta, v_{s},|\lambda|,|\kappa|, \operatorname{Re} A_{\kappa}}_{\overline{\mathrm{DR}}}$,

while in the second scheme $\operatorname{Re} A_{\lambda}$ is an input parameter renormalized as a $\overline{\mathrm{DR}}$ parameter and the charged Higgs mass is a derived quantity,

$\underbrace{t_{h_{d}}, t_{h_{u}}, t_{h_{s}}, t_{a_{d}}, t_{a_{s}}, M_{W}^{2}, M_{Z}^{2}, e}_{\text {on-shell }}, \underbrace{\tan \beta, v_{s},|\lambda|,|\kappa|, \operatorname{Re} A_{\lambda}, \operatorname{Re} A_{\kappa}}_{\overline{\mathrm{DR}}}$. 
We use the dimensional reduction scheme [20,21], which up to one-loop order has been proven to be SUSY conserving, to regularize the UV-divergent tadpoles and unrenormalized self-energies. In particular, the manipulation of the Dirac matrices is done in 4-dimensional space-time while the tensor reduction and one-loop integrals are computed in $D$ dimensional space-time, $D=4-2 \epsilon$. The UV divergence is then parameterized by the pole $1 / \epsilon$ in the limit $\epsilon \rightarrow 0$, i.e. in the physical $D=4$ space-time dimension. For the definition of the one-loop OS counterterms we refer the reader to Ref. [16]. For the counterterms of the $\overline{\mathrm{DR}}$ parameters, they are proportional to the combination $1 / \epsilon-\gamma_{E}+\log (4 \pi)$, where $\gamma_{E}$ is the Euler-Mascheroni constant. Therefore, they do not contribute to the final physical results. We, however, keep them to ensure the cancellation of the UV divergences as it is a powerful check of our implementation of the renormalization procedure. We define these counterterms solely in the Higgs sector by requiring that all renormalized self-energies of the Higgs bosons be finite. This is different with respect to the definition in Ref. [16] where the chargino and neutralino sectors were used. The numerical results between the two definitions are identical, however. We renormalize the Higgs fields in the $\overline{\mathrm{DR}}$ scheme as described in Ref. [16] at one-loop level, and in Refs. [17,18] at two-loop order $\mathcal{O}\left(\alpha_{t} \alpha_{s}\right)$ and $\mathcal{O}\left(\alpha_{t}^{2}\right)$, respectively.

\subsubsection{The chargino and neutralino sector}

The chargino and neutralino sectors are described by fourteen real parameters: $M_{W}, M_{Z}, \tan \beta, v_{s}, \varphi_{s}, \varphi_{u},|\lambda|, \varphi_{\lambda},|\kappa|$, $\varphi_{\kappa},\left|M_{1}\right|, \varphi_{M_{1}},\left|M_{2}\right|, \varphi_{M_{2}}$. Since the first ten of these already appear in the Higgs sector, there remains to define the renormalization conditions for the four parameters $\left|M_{1}\right|, \varphi_{M_{1}}$, $\left|M_{2}\right|, \varphi_{M_{2}}{ }^{2}$ There are no physical renormalization conditions to fix the counterterms of the phases $\varphi_{M_{1}}, \varphi_{M_{2}}$. It has been found in Ref. [16] that the complex phases of $M_{1}$ and $M_{2}$ do not need to be renormalized at the one-loop level. We verified this statement in our computation. ${ }^{3}$ In addition, we have to renormalize the chargino and neutralino fields in order to obtain finite self-energies. In the literature there exist two descriptions for the introduction of wave function renormalization (WFR) constants. In the Espriu-ManzanoTalavera (EMT) description two independent renormalization constants were introduced for incoming and outgoing fermions [22-24]. Thanks to more degrees of freedom one can keep contributions arising from absorptive parts of the loop integrals and eliminate completely the mixing self-

\footnotetext{
${ }^{2}$ In the case of the MSSM one needs to renormalize three complex parameters $M_{1}, M_{2}, \mu$.

3 The same holds true in the complex MSSM [22] The three complex phases of $M_{1}, M_{2}$ and $\mu$ do not need to be renormalized in order to render all Green functions finite.
}

energies thereby fulfilling the standard OS conditions. However, the hermicity of the renormalized Lagrangian is not satisfied any more. In the Denner description [25], one WFR constant was used instead. It preserves the hermicity constraint, but the absorptive part of the loop integral must be eliminated. We want to investigate the effect of the absorptive part and therefore apply both descriptions. In the following we will derive the formulae in the EMT method. From these formulae, one can easily obtain the ones in the Denner description. The bare parameters and fields are replaced by the renormalized ones and the corresponding counterterms as

$$
\begin{aligned}
M_{1} & \rightarrow M_{1}+\delta\left|M_{1}\right| e^{i \varphi_{M_{1}}}, \\
M_{2} & \rightarrow M_{2}+\delta\left|M_{2}\right| e^{i \varphi_{M_{2}}}, \\
P_{L} \tilde{\chi}_{i}^{+} & \rightarrow\left(1+\frac{1}{2} \delta Z_{L}^{\tilde{\chi}^{+}}\right)_{i j} P_{L} \tilde{\chi}_{j}^{+}, \\
\overline{\tilde{\chi}_{i}^{+}} P_{L} & \rightarrow \overline{\tilde{\chi}_{j}^{+}}\left(1+\frac{1}{2} \delta \bar{Z}_{R}^{\tilde{\chi}^{+}}\right)_{j i} P_{L}, \\
P_{R} \tilde{\chi}_{i}^{+} & \rightarrow\left(1+\frac{1}{2} \delta Z_{R}^{\tilde{\chi}^{+}}\right)_{i j} P_{R} \tilde{\chi}_{j}^{+}, \\
\tilde{\tilde{\chi}}_{i}^{+} P_{R} & \rightarrow \overline{\tilde{\chi}_{j}^{+}}\left(1+\frac{1}{2} \delta \bar{Z}_{L}^{\tilde{\chi}^{+}}\right)_{j i} P_{R}, \\
P_{L} \tilde{\chi}_{k}^{0} & \rightarrow\left(1+\frac{1}{2} \delta Z_{L}^{\tilde{\chi}^{0}}\right)_{k l} P_{L} \tilde{\chi}_{l}^{0}, \\
\overline{\tilde{\chi}_{k}^{0}} P_{L} & \rightarrow \overline{\tilde{\chi}_{l}^{0}}\left(1+\frac{1}{2} \delta \bar{Z}_{R}^{\tilde{\chi}^{0}}\right)_{l k} P_{L}, \\
P_{R} \tilde{\chi}_{k}^{0} & \rightarrow\left(1+\frac{1}{2} \delta Z_{R}^{\tilde{\chi}^{0}}\right)_{k l} P_{R} \tilde{\chi}_{l}^{0}, \\
\overline{\tilde{\chi}_{k}^{0}} P_{R} & \rightarrow \frac{1}{\tilde{\chi}_{l}^{0}}\left(1+\frac{1}{2} \delta \bar{Z}_{L}^{0}\right)_{l k} P_{R},
\end{aligned}
$$

where $i, j=1,2$ and $k, l=1, \ldots, 5$. Since the neutralinos are Majorana fermions we have

$\delta Z_{R}^{\tilde{\chi}^{0}}=\left(\delta \bar{Z}_{L}^{\tilde{\chi}^{0}}\right)^{T} \quad$ and $\quad \delta Z_{L}^{\tilde{\chi}^{0}}=\left(\delta \bar{Z}_{R}^{\tilde{\chi}^{0}}\right)^{T}$.

Note that we do not need to renormalize the rotation matrices $U, V$ and $N$ because their counterterms are redundant. They always appear in combination with WFR constants. One can therefore always redefine the WFR constants to absorb the counterterms of the rotation matrices, as shown in Ref. [26]. In general, the renormalized self-energies $\hat{\Sigma}$ of the fermions can be cast into the following form, $c f$. Ref. [25],

$$
\begin{aligned}
\hat{\Sigma}_{i j}(p)= & p \hat{\Sigma}_{i j}^{L}\left(p^{2}\right) P_{L}+p \hat{\Sigma}_{i j}^{R}\left(p^{2}\right) P_{R}+\hat{\Sigma}_{i j}^{L s}\left(p^{2}\right) P_{L} \\
& +\hat{\Sigma}_{i j}^{R s}\left(p^{2}\right) P_{R},
\end{aligned}
$$

with

$$
\begin{aligned}
& \hat{\Sigma}_{i j}^{L}\left(p^{2}\right)=\Sigma_{i j}^{L}\left(p^{2}\right)+\frac{1}{2}\left(\delta Z_{L}+\delta \bar{Z}_{L}\right)_{i j}, \\
& \hat{\Sigma}_{i j}^{R}\left(p^{2}\right)=\Sigma_{i j}^{R}\left(p^{2}\right)+\frac{1}{2}\left(\delta Z_{R}+\delta \bar{Z}_{R}\right)_{i j},
\end{aligned}
$$




$$
\begin{aligned}
\hat{\Sigma}_{i j}^{L s}\left(p^{2}\right)= & \Sigma_{i j}^{L s}\left(p^{2}\right) \\
& -\left(\frac{1}{2}\left(\delta \bar{Z}_{R}\right)_{i j} m_{\tilde{\chi}_{j}}+\frac{1}{2}\left(\delta Z_{L}\right)_{i j} m_{\tilde{\chi}_{i}}+\left(\delta M_{\tilde{\chi}}^{\text {tree }}\right)_{i j}\right), \\
\hat{\Sigma}_{i j}^{R s}\left(p^{2}\right)= & \Sigma_{i j}^{R s}\left(p^{2}\right) \\
& -\left(\frac{1}{2}\left(\delta \bar{Z}_{L}\right)_{i j} m_{\tilde{\chi}_{j}}+\frac{1}{2}\left(\delta Z_{R}\right)_{i j} m_{\tilde{\chi}_{i}}+\left(\delta M_{\tilde{\chi}}^{\text {tree }}\right)_{i j}^{\dagger}\right),
\end{aligned}
$$

where $\Sigma_{i j}(p)$ denotes the unrenormalized self-energy of the transition $\tilde{\chi}_{i}^{+} \rightarrow \tilde{\chi}_{j}^{+}, i, j=1,2$, for the charginos and $\tilde{\chi}_{i}^{0} \rightarrow \tilde{\chi}_{j}^{0}, i, j=1, \ldots, 5$, for the neutralinos. For the charginos, the tree-level mass matrix $M_{\tilde{\chi}}^{\text {tree }}$ and its countert$\operatorname{erm} \delta M_{\tilde{\chi}}^{\text {tree }}$ are given by

$M_{\tilde{\chi}}^{\text {tree }}=U^{*} M_{C} V^{\dagger}, \quad \delta M_{\tilde{\chi}}^{\text {tree }}=U^{*} \delta M_{C} V^{\dagger}$

and for the neutralinos by

$M_{\tilde{\chi}}^{\text {tree }}=N^{*} M_{N} N^{\dagger}, \quad \delta M_{\tilde{\chi}}^{\text {tree }}=N^{*} \delta M_{N} N^{\dagger}$.

In the following, we will discuss the OS conditions for the general fermion fields $\tilde{\chi}_{i}$ having the tree-level masses $m_{\tilde{\chi}_{i}}$. The renormalized fermion propagator matrix is given by

$\hat{S}(p)=-\hat{\Gamma}(p)^{-1}$,

where the renormalized one-particle irreducible (1PI) twopoint functions $\hat{\Gamma}$ are related to the renormalized self-energies as

$\hat{\Gamma}_{i j}(p)=i \delta_{i j}\left(p-m_{\tilde{\chi}_{i}}\right)+i \hat{\Sigma}_{i j}(p)$.

The propagator matrix has complex poles at $\mathcal{M}_{\tilde{\chi}_{i}}^{2}=M_{\tilde{\chi}_{i}}^{2}-$ $i M_{\tilde{\chi}_{i}} \Gamma_{\tilde{\chi}_{i}}$, where $\Gamma_{\tilde{\chi}_{i}}$ denotes the decay widths. In the OS scheme we require that the tree-level masses are equal to the physical masses, the mixing terms are vanishing at the poles and that the residues of the propagators are unity, ${ }^{4}$

$$
\begin{aligned}
\left.\operatorname{Re} \hat{\Sigma}_{i i}(p) \tilde{\chi}_{i}\right|_{p^{2}=m_{\tilde{\chi}_{i}}^{2}} & =0, \\
\left.\hat{\Sigma}_{i j}(p) \tilde{\chi}_{j}\right|_{p^{2}=m_{\tilde{\chi}_{j}}^{2}} & =0, \\
\left.\tilde{\chi}_{i} \hat{\Sigma}_{i j}(p)\right|_{p^{2}=m_{\tilde{\chi}_{i}}^{2}} & =0, \quad i \neq j
\end{aligned}
$$

\footnotetext{
${ }^{4}$ In the Denner description, $\hat{\Sigma}_{i i}$ in Eq. (3.21), $\hat{\Sigma}_{i j}$ in Eq. (3.22) and $\hat{\Gamma}_{i i}$ in Eq. (3.23) are replaced by $\widetilde{\operatorname{Re}} \hat{\Sigma}_{i i}, \widetilde{\operatorname{Re}} \hat{\Sigma}_{i j}$ and $\widetilde{\operatorname{Re}} \hat{\Gamma}_{i i}$, respectively. $\widetilde{\operatorname{Re}}$ means that one takes only the real part of the loop integrals but leaves the couplings unaffected.
}

$\lim _{p^{2} \rightarrow m_{\tilde{\chi}_{i}}} \frac{1}{p-m_{\tilde{\chi}_{i}}} \operatorname{Re} \hat{\Gamma}_{i i} \tilde{\chi}_{i}=i \tilde{\chi}_{i}$

$\lim _{p^{2} \rightarrow m_{\tilde{\chi}_{i}}} \overline{\tilde{\chi}_{i}} \operatorname{Re} \hat{\Gamma}_{i i} \frac{1}{p-m_{\tilde{\chi}_{i}}}=i \overline{\tilde{\chi}_{i}}$.

In addition, we require the chiral structure to vanish in the OS limit, ${ }^{5}$

$\hat{\Sigma}_{i i}^{L}\left(m_{\tilde{\chi}_{i}}^{2}\right)=\hat{\Sigma}_{i i}^{R}\left(m_{\tilde{\chi}_{i}}^{2}\right), \quad \hat{\Sigma}_{i i}^{L s}\left(m_{\tilde{\chi}_{i}}^{2}\right)=\hat{\Sigma}_{i i}^{R s}\left(m_{\tilde{\chi}_{i}}^{2}\right)$.

Applying the decomposition in Eq. (3.12) and the tree-level relations

$\left(p-m_{\tilde{\chi}_{i}}\right) \tilde{\chi}_{i}=0, \quad \overline{\tilde{\chi}_{i}}\left(p+m_{\tilde{\chi}_{i}}\right)=0$,

one obtains the mass counterterms $\delta m_{\tilde{\chi}_{i}}=\operatorname{Re}\left(\delta M_{\tilde{\chi}}^{\text {tree }}\right)_{i i}$, with

$$
\begin{aligned}
\operatorname{Re}\left(\delta M_{\tilde{\chi}}^{\text {tree }}\right)_{i i}= & \frac{1}{2}\left(m_{\tilde{\chi}_{i}} \operatorname{Re} \Sigma_{i i}^{L}\left(m_{\tilde{\chi}_{i}}^{2}\right)+m_{\tilde{\chi}_{i}} \operatorname{Re} \Sigma_{i i}^{R}\left(m_{\tilde{\chi}_{i}}^{2}\right)\right. \\
& \left.+\operatorname{Re} \Sigma_{i i}^{L s}\left(m_{\tilde{\chi}_{i}}^{2}\right)+\operatorname{Re} \Sigma_{i i}^{R s}\left(m_{\tilde{\chi}_{i}}^{2}\right)\right),
\end{aligned}
$$

the off-diagonal wave function renormalization constants, $\delta Z_{L / R, i j}, \delta \bar{Z}_{L / R, i j},{ }^{6,7}$

$$
\begin{aligned}
\delta Z_{L, i j}= & \frac{2}{m_{\tilde{\chi}_{i}}^{2}-m_{\tilde{\chi}_{j}}^{2}} \\
& \times\left[m_{\tilde{\chi}_{j}}^{2} \Sigma_{i j}^{L}\left(m_{\tilde{\chi}_{j}}^{2}\right)+m_{\tilde{\chi}_{i}} m_{\tilde{\chi}_{j}} \Sigma_{i j}^{R}\left(m_{\tilde{\chi}_{j}^{2}}\right)\right. \\
& +m_{\tilde{\chi}_{i}} \Sigma_{i j}^{L s}\left(m_{\tilde{\chi}_{j}}^{2}\right)+m_{\tilde{\chi}_{j}} \Sigma_{i j}^{R s}\left(m_{\tilde{\chi}_{j}}^{2}\right) \\
& \left.-m_{\tilde{\chi}_{j}}\left(\delta M_{\tilde{\chi}}^{\mathrm{tree}}\right)_{i j}^{\dagger}-m_{\tilde{\chi}_{i}}\left(\delta M_{\tilde{\chi}}^{\mathrm{tree}}\right)_{i j}\right], \\
\delta Z_{R, i j}= & \frac{2}{m_{\tilde{\chi}_{i}}^{2}-m_{\tilde{\chi}_{j}}^{2}} \\
& \times\left[m_{\tilde{\chi}_{j}}^{2} \Sigma_{i j}^{R}\left(m_{\tilde{\chi}_{j}}^{2}\right)+m_{\tilde{\chi}_{i}} m_{\tilde{\chi}_{j}} \Sigma_{i j}^{L}\left(m_{\tilde{\chi}_{j}}^{2}\right)\right. \\
& +m_{\tilde{\chi}_{i}} \Sigma_{i j}^{R s}\left(m_{\tilde{\chi}_{j}}^{2}\right)+m_{\tilde{\chi}_{j}} \Sigma_{i j}^{L s}\left(m_{\tilde{\chi}_{j}}^{2}\right) \\
& \left.-m_{\tilde{\chi}_{i}}\left(\delta M_{\tilde{\chi}^{\mathrm{t}}}^{\mathrm{tree}}\right)_{i j}^{\dagger}-m_{\tilde{\chi}_{j}}\left(\delta M_{\tilde{\chi}}^{\mathrm{tree}}\right)_{i j}\right], \\
\delta \bar{Z}_{L, i j}= & \delta Z_{L, i j}\left(m_{\tilde{\chi}_{j}}^{2} \leftrightarrow m_{\tilde{\chi}_{i}}^{2}\right), \\
\delta \bar{Z}_{R, i j}= & \delta Z_{R, i j}\left(m_{\tilde{\chi}_{j}}^{2} \leftrightarrow m_{\tilde{\chi}_{i}}^{2}\right)
\end{aligned}
$$

\footnotetext{
5 If we use the Denner OS conditions in Ref. [25] then these relations are automatically satisfied for the real parts only.

${ }^{6}$ In the Denner description $\Sigma_{i j}^{L / R / L s / R s}$ are replaced by $\widetilde{\operatorname{Re}} \Sigma_{i j}^{L / R / L s / R s}$.

${ }^{7}$ Note that the $\delta \bar{Z}$ are obtained from $\delta Z$ by interchanging the $m_{\tilde{\chi}}^{2}$ of the electroweakinos, but not the $m_{\tilde{\chi}}$.
} 
and the diagonal wave function renormalization constants ${ }^{8}$

$$
\begin{aligned}
\delta Z_{L, i i}= & -\Sigma_{i i}^{L}\left(m_{\tilde{\chi}_{i}}^{2}\right)-m_{\tilde{\chi}_{i}} \frac{\partial}{\partial p^{2}} \\
& \times\left.\left[m_{\tilde{\chi}_{i}} \Sigma_{i i}^{L}\left(p^{2}\right)+m_{\tilde{\chi}_{i}} \Sigma_{i i}^{R}\left(p^{2}\right)+\Sigma_{i i}^{L s}\left(p^{2}\right)+\Sigma_{i i}^{R s}\left(p^{2}\right)\right]\right|_{p^{2}=m_{\tilde{\chi}_{i}}^{2}} \\
& +a \\
\delta Z_{R, i i}= & -\Sigma_{i i}^{R}\left(m_{\tilde{\chi}_{i}}^{2}\right)-m_{\tilde{\chi}_{i}} \frac{\partial}{\partial p^{2}} \\
& \times\left.\left[m_{\tilde{\chi}_{i}} \Sigma_{i i}^{L}\left(p^{2}\right)+m_{\tilde{\chi}_{i}} \Sigma_{i i}^{R}\left(p^{2}\right)+\Sigma_{i i}^{L s}\left(p^{2}\right)+\Sigma_{i i}^{R s}\left(p^{2}\right)\right]\right|_{p^{2}=m_{\tilde{\chi}_{i}}^{2}} \\
& +b+a \\
\delta \bar{Z}_{L, i i}= & \delta Z_{L, i i}-2 a \\
\delta \bar{Z}_{R, i i}= & \delta Z_{R, i i}-2 b-2 a,
\end{aligned}
$$

where

$$
\begin{aligned}
b=- & \frac{1}{m_{\tilde{\chi}_{i}}}\left[\Sigma_{i i}^{L s}\left(m_{\tilde{\chi}_{i}}^{2}\right)-\Sigma_{i i}^{R s}\left(m_{\tilde{\chi}_{i}}^{2}\right)-\left(\delta M_{\tilde{\chi}}^{\text {tree }}\right)_{i i}\right. \\
& \left.+\left(\delta M_{\tilde{\chi}}^{\text {tree }}\right)_{i i}^{*}\right] \\
a=- & \frac{b}{2} .
\end{aligned}
$$

Our results coincide with those given in Ref. [22]. The above wave function renormalization constants have been chosen such that for all fermions the mixing terms are cancelled and the correct propagators are produced at the tree-level mass values. Note that in case we do not have enough parameters to renormalize all fermions on-shell, only some of these fermions satisfy OS conditions, i.e. their tree-level masses are equal to the physical masses. The remaining fermions have loop-corrected masses. This is the case for the electroweakino sector. Given the fact that we have already fixed the renormalization scheme for the Higgs sector, only the two gaugino masses $M_{1}$ and $M_{2}$ remain to be renormalized, while we have seven masses ( 5 neutralino and 2 chargino masses), so that only two of them can be set OS. The remaining 5 particles receive loop-corrections to their masses. At loop level the mixing between fermions is in general not vanishing any more, and the residues of the propagators are not unity. These effects should be taken into account if the loop corrections to the masses are large. This is not the case for the renormalization schemes chosen here, therefore we neglect these effects.

In the chargino and neutralino sector hence only the two counterterms $\delta\left|M_{1}\right|$ and $\delta\left|M_{2}\right|$ remain to be determined. There are 20 different ways to choose two out of the seven masses for the OS conditions. We will consider here two different schemes. In the first scheme (OS1), we require the masses of the wino-like chargino $\tilde{\chi}_{i}^{+}$and the bino-like neutralino $\tilde{\chi}_{k}^{0}$ to be OS. The bino-like neutralino is sensitive to

${ }^{8}$ In the Denner description $a=b=0$ and the $\Sigma_{i i}^{L / R / L s / R s}$ are replaced by $\widetilde{\operatorname{Re}} \Sigma_{i i}^{L / R / L s / R s}$.
$M_{1}$ while the wino-like chargino is sensitive to $M_{2} \cdot{ }^{9}$ Note that we do not choose the chargino and neutralino by referring to a fixed index order since they may not be sensitive to $M_{1}$ or $M_{2}$. This can then lead to numerical instability, as was found in the MSSM [26-29] and in [7,8] for the NMSSM. We denote the tree-level masses for the neutralinos (charginos) by a small letter $m_{\tilde{x}_{i}^{0(+)}}$, and the loop corrected masses by a capital letter $M_{\tilde{\chi}_{i}^{0(+)}}$. In the OS scheme the tree-level masses are equal to the loop-corrected ones. We define the counterterm mass matrices of the chargino and neutralino sector in the interaction basis, $\delta M_{N}$ and $\delta M_{C}$, through

$M_{N} \rightarrow M_{N}+\delta M_{N}$ and $M_{C} \rightarrow M_{C}+\delta M_{C}$,

with the neutralino mass matrix given in Eq. (2.13) and the chargino mass matrix in Eq. (2.18). The counterterms for $M_{1}$ and $M_{2}$ are then given by

$$
\begin{aligned}
\delta\left|M_{2}\right|= & \frac{1}{\operatorname{Re}\left[U_{i 1}^{*} V_{i 1}^{*} e^{\left.i \phi_{M_{2}}\right]}\right.} \\
& \times\left[\frac{1}{2} m_{\tilde{\chi}_{i}^{+}}\left(\operatorname{Re} \Sigma_{i i}^{\tilde{\chi}_{i}^{+}, L}\left(m_{\tilde{\chi}_{i}^{+}}^{2}\right)+\operatorname{Re} \Sigma_{i i}^{\tilde{\chi}_{i}^{+}, R}\left(m_{\tilde{\chi}_{i}^{+}}^{2}\right)\right)\right. \\
& +\frac{1}{2} \operatorname{Re} \Sigma_{i i}^{\tilde{\chi}_{i}^{+}, L s}\left(m_{\tilde{\chi}_{i}^{+}}^{2}\right)+\frac{1}{2} \operatorname{Re} \Sigma_{i i}^{\tilde{\chi}_{i}^{+}, R s}\left(m_{\tilde{\chi}_{i}^{+}}^{2}\right) \\
& \left.-\left.\operatorname{Re}\left[U^{*} \delta M_{C} V^{\dagger}\right]_{i i}\right|_{\delta M_{2}=0}\right] \\
\delta\left|M_{1}\right|= & \frac{1}{\operatorname{Re}\left[N_{k 1}^{*} N_{k 1}^{*} e^{\left.i \phi_{M_{1}}\right]}\right.} \\
& \times\left[\frac{1}{2} m_{\tilde{\chi}_{k}^{0}}\left(\operatorname{Re} \Sigma_{k k}^{\tilde{\chi}_{k}^{0}, L}\left(m_{\tilde{\chi}_{k}^{0}}^{2}\right)+\operatorname{Re} \Sigma_{k k}^{\chi_{k}^{0}, R}\left(m_{\tilde{\chi}_{k}^{0}}^{2}\right)\right)\right. \\
& +\frac{1}{2} \operatorname{Re} \Sigma_{k k}^{\tilde{\chi}_{k}^{0}, L s}\left(m_{\tilde{\chi}_{k}^{0}}^{2}\right)+\frac{1}{2} \operatorname{Re} \Sigma_{k k}^{\tilde{\chi}_{k}^{0}, R s}\left(m_{\tilde{\chi}_{k}^{0}}^{2}\right) \\
& \left.-\left.\operatorname{Re}\left[N^{*} \delta M_{N} N^{\dagger}\right]_{k k}\right|_{\delta M_{1}=0}\right] .
\end{aligned}
$$

In the second scheme (OS2), we use the masses of the bino-like neutralino, denoted by $\tilde{\chi}_{k}^{0}$, and of the wino-like neutralino, denoted by $\tilde{\chi}_{l}^{0}$, as inputs. The renormalization conditions for their OS masses are given by

$$
\begin{aligned}
& \left.\hat{\Sigma}_{k k}^{\tilde{\chi}_{i}^{0}}\left(p^{2}\right) \tilde{\chi}_{k}^{0}\right|_{p^{2}=m_{\tilde{\chi}_{k}^{0}}^{2}}=0 \\
& \left.\hat{\Sigma}_{l l}^{\tilde{\chi}_{i}^{0}}\left(p^{2}\right) \tilde{\chi}_{l}^{0}\right|_{p^{2}=m_{\tilde{\chi}_{l}^{0}}^{2}}=0 .
\end{aligned}
$$

This results in the two solutions for the counterterms $\delta\left|M_{1}\right|$ and $\delta\left|M_{2}\right|$,

\footnotetext{
${ }^{9}$ We find the bino-like neutralino (wino-like chargino) by looking at the tree-level rotation matrix $N(U / V)$. The position of the maximal value in the list $\left\{\left|N_{11}\right|, \ldots,\left|N_{51}\right|\right\}$ is identical to the position of the bino-like neutralino. For the wino-like neutralino position we look for the position of the maximal value in the list $\left\{\left|N_{12}\right|, \ldots,\left|N_{52}\right|\right\}$. The wino-like chargino is found by identifying the position of the maximal value of $\left\{\left|U_{11}\right|,\left|U_{21}\right|\right\}$.
} 
$\begin{aligned} \delta\left|M_{1}\right| & =\frac{a_{1} \operatorname{Re}\left[N_{l 2}^{*} N_{l 2}^{*} e^{i \phi_{M_{2}}}\right]-a_{2} \operatorname{Re}\left[N_{k 2}^{*} N_{k 2}^{*} e^{i \phi_{M_{2}}}\right]}{\operatorname{Re}\left[N_{k 1}^{*} N_{k 1}^{*} e^{i \phi_{M_{1}}}\right] \operatorname{Re}\left[N_{l 2}^{*} N_{l 2}^{*} e^{i \phi_{M_{2}}}\right]-\operatorname{Re}\left[N_{k 2}^{*} N_{k 2}^{*} e^{i \phi_{M_{2}}}\right] \operatorname{Re}\left[N_{l 1}^{*} N_{l 1}^{*} e^{i \phi_{M_{1}}}\right]} \\ \delta\left|M_{2}\right| & =-\frac{a_{1} \operatorname{Re}\left[N_{l 1}^{*} N_{l 1}^{*} e^{i \phi_{M_{1}}}\right]-a_{2} \operatorname{Re}\left[N_{k 1}^{*} N_{k 1}^{*} e^{i \phi_{M_{1}}}\right]}{\operatorname{Re}\left[N_{k 1}^{*} N_{k 1}^{*} e^{i \phi_{M_{1}}}\right] \operatorname{Re}\left[N_{l 2}^{*} N_{l 2}^{*} e^{i \phi_{M_{2}}}\right]-\operatorname{Re}\left[N_{k 2}^{*} N_{k 2}^{*} e^{i \phi_{M_{2}}}\right] \operatorname{Re}\left[N_{l 1}^{*} N_{l 1}^{*} e^{i \phi_{M_{1}}}\right]}\end{aligned}$

with

$$
\begin{aligned}
a_{1}= & {\left[\frac { 1 } { 2 } \left(m_{\tilde{\chi}_{k}^{0}}\left(\operatorname{Re} \Sigma_{k k}^{\tilde{\chi}_{k}^{0}, L}\left(m_{\tilde{\chi}_{k}^{0}}^{2}\right)+\operatorname{Re} \Sigma_{k k}^{\tilde{\chi}_{k}^{0}, R}\left(m_{\tilde{\chi}_{k}^{0}}^{2}\right)\right)\right.\right.} \\
& \left.+\operatorname{Re} \Sigma_{k k}^{\tilde{\chi}_{k}^{0}, L s}\left(m_{\tilde{\chi}_{k}^{0}}^{2}\right)+\operatorname{Re} \Sigma_{k k}^{\tilde{\chi}_{k}^{0}, R s}\left(m_{\tilde{\chi}_{k}^{0}}^{2}\right)\right) \\
& \left.-\left.\operatorname{Re}\left(N^{*} \delta M_{N} N^{\dagger}\right)_{k k}\right|_{\delta M_{1}=\delta M_{2}=0}\right] \\
a_{2}= & {\left[\frac { 1 } { 2 } \left(m_{\tilde{\chi}_{l}^{0}}\left(\operatorname{Re} \Sigma_{l l}^{\tilde{\chi}_{l}^{0}, L}\left(m_{\tilde{\chi}_{l}^{0}}^{2}\right)+\operatorname{Re} \Sigma_{l l}^{\tilde{\chi}_{l}^{0}, R}\left(m_{\tilde{\chi}_{l}^{0}}^{2}\right)\right)\right.\right.} \\
& \left.+\operatorname{Re} \Sigma_{l l}^{\tilde{\chi}_{l}^{0}, L s}\left(m_{\tilde{\chi}_{l}^{0}}^{2}\right)+\operatorname{Re} \Sigma_{l l}^{\tilde{\chi}_{l}^{0}, R s}\left(m_{\tilde{\chi}_{l}^{0}}^{2}\right)\right) \\
& \left.-\left.\operatorname{Re}\left(N^{*} \delta M_{N} N^{\dagger}\right)_{l l}\right|_{\delta M_{1}=\delta M_{2}=0}\right] .
\end{aligned}
$$

For the field renormalization constants of the charginos and neutralinos, we impose the OS conditions for the treelevel masses. Besides the two OS schemes, we will also adopt the $\overline{\mathrm{DR}}$ renormalization scheme for $M_{1}$ and $M_{2}$, while for the field renormalization constants we use the OS conditions.

\subsubsection{The squark sector}

We consider here only the third-generation squarks. The results for the first- and second-generation squarks are obtained analogously. ${ }^{10}$ There are seven parameters to be renormalized in this sector,

$m_{t}, m_{b}, m_{\tilde{Q}_{3}}^{2}, m_{\tilde{t}_{R}}^{2}, m_{\tilde{b}_{R}}^{2}, A_{t}, A_{b}$,

where $A_{t}, A_{b}$ are complex and the mass terms are real. We denote their corresponding counterterms as

$\delta m_{t}, \delta m_{b}, \delta m_{\tilde{Q}_{3}}^{2}, \delta m_{\tilde{t}_{R}}^{2}, \delta m_{\tilde{b}_{R}}^{2}, \delta A_{t}, \delta A_{b}$,

and define the squark-mass counterterm matrices as

$M_{\tilde{q}} \rightarrow M_{\tilde{q}}+\delta M_{\tilde{q}}$,

with $M_{\tilde{q}}$ given in Eq. (2.23) for the stops and in Eq. (2.24) for the sbottoms. The renormalization of the remaining parameters appearing in the squark mass matrices has been specified

\footnotetext{
${ }^{10}$ If the light quark masses are set to zero, the mixing between left- and right-handed squarks vanishes. In this case, it is not required to renormalize the quark masses and the trilinear couplings. Equations (3.55), (3.56), (3.67) are still usable by removing the contribution that then becomes zero. At present, the SUSY-QCD and SUSY-EW corrections are not included in the decays into light squarks in our code.
}

in the renormalization of the Higgs sector, more specifically see Refs. [15-18].

We have to renormalize the squark fields in order to make the squark self-energies finite. Here we use both the EMT and the Denner description. For the EMT description we have to introduce two separate WFR constants, one for the particle and one for the anti-particle. We introduce the squark WFR constants for the particles and anti-particles in the mass eigenstate basis as ${ }^{11}$

$$
\begin{aligned}
& \left(\begin{array}{c}
\tilde{q}_{1} \\
\tilde{q}_{2}
\end{array}\right) \rightarrow\left(1+\frac{1}{2} \delta Z_{\tilde{q}}\right)\left(\begin{array}{c}
\tilde{q}_{1} \\
\tilde{q}_{2}
\end{array}\right), \\
& \left(\begin{array}{c}
\tilde{q}_{1} \\
\tilde{q}_{2}
\end{array}\right)^{\dagger} \rightarrow\left(\begin{array}{l}
\tilde{q}_{1} \\
\tilde{q}_{2}
\end{array}\right)^{\dagger}\left(1+\frac{1}{2} \delta \bar{Z}^{\tilde{q}}\right) .
\end{aligned}
$$

The renormalized self-energies in the mass eigenstate basis are given by $(i, j=1,2)$

$$
\begin{aligned}
\hat{\Sigma}_{i j}^{\tilde{q}}\left(p^{2}\right)= & \Sigma_{i j}^{\tilde{q}}\left(p^{2}\right)+\frac{1}{2}\left(\delta \bar{Z}_{i j}^{\tilde{q}}+\delta Z_{i j}^{\tilde{q}}\right) p^{2} \\
& -\frac{1}{2}\left(\delta \bar{Z}_{i j}^{\tilde{q}} m_{\tilde{q}_{j}}^{2}+m_{\tilde{q}_{i}}^{2} \delta Z_{i j}^{\tilde{q}}\right) \\
& -\left(U_{\tilde{q}} \delta M_{\tilde{q}} U_{\tilde{q}}^{\dagger}\right)_{i j},
\end{aligned}
$$

where we denote by $\Sigma_{i j}^{\tilde{q}}$ the unrenormalized self-energies for the $\tilde{q}_{i}^{*} \rightarrow \tilde{q}_{j}^{*}$ transition. ${ }^{12}$ In the following, we give the OS counterterms. The $\overline{\mathrm{DR}}$ counterterms are then easily obtained by taking only the divergent parts of the corresponding OS counterterms.

Applying the decomposition of the fermionic self-energies as given in Eq. (3.12), the mass counterterm in the OS scheme for the top and bottom quark, respectively, is given by $(q=t, b)^{13}$

$$
\begin{aligned}
\delta m_{q}= & \frac{1}{2} \operatorname{Re} \\
& \times\left\{\left(\Sigma_{q}^{L}\left(m_{q}^{2}\right)+\Sigma_{q}^{R}\left(m_{q}^{2}\right)\right) m_{q}+\Sigma_{q}^{L s}\left(m_{q}^{2}\right)+\Sigma_{q}^{R s}\left(m_{q}^{2}\right)\right\} .
\end{aligned}
$$

\footnotetext{
${ }^{11}$ In the Denner description, we have $\delta \bar{Z}_{\tilde{q}}=\delta Z_{\tilde{q}}^{\dagger}$.

12 Note that in the real NMSSM the unrenormalized self-energies for the $\tilde{q}_{i}^{*} \rightarrow \tilde{q}_{j}^{*}$ transition and for the $\tilde{q}_{i} \rightarrow \tilde{q}_{j}$ transition are identical. They are different, however, in the complex case.

${ }^{13}$ In the Denner description, Re is replaced by $\widetilde{\mathrm{Re}}$.
} 
The OS conditions for the scalar renormalized self-energies are $(i, j=1,2)^{14}$

$$
\begin{aligned}
& \operatorname{Re} \hat{\Sigma}_{i i}^{\tilde{q}}\left(m_{\tilde{q}_{i}}^{2}\right)=0 \\
& \hat{\Sigma}_{i j}^{\tilde{q}}\left(m_{\tilde{q}_{i}}^{2}\right)=0, \quad \hat{\Sigma}_{i j}^{\tilde{q}}\left(m_{\tilde{q}_{j}}^{2}\right)=0, \quad i \neq j \\
& \left.\frac{\partial \operatorname{Re} \hat{\Sigma}_{i i}^{\tilde{q}}\left(p^{2}\right)}{\partial p^{2}}\right|_{p^{2}=m_{\tilde{q}_{i}}^{2}}=0 .
\end{aligned}
$$

We apply the conditions in Eqs. (3.52) and (3.53) for the top squark to determine $\delta A_{t}, \delta m_{\tilde{Q}_{3}}^{2}$ and $\delta m_{\tilde{t}_{R}}^{2},{ }^{15}$

$$
\begin{aligned}
\delta m_{\tilde{Q}_{3}}^{2}= & \left|U_{11}^{\tilde{t}}\right|^{2} \delta m_{\tilde{t}_{1}}^{2}+\left|U_{12}^{\tilde{t}}\right|^{2} \delta m_{\tilde{t}_{2}}^{2}+U_{21}^{\tilde{t}} U_{11}^{\tilde{t} *} \delta Y \\
& +U_{11}^{\tilde{t}} U_{21}^{\tilde{t} *}(\delta Y)^{*}-2 m_{t} \delta m_{t} \\
& +\frac{2}{3} \sin \beta \cos ^{3} \beta M_{Z}^{2}\left(3-4 \sin ^{2} \theta_{W}\right) \delta \tan \beta \\
& +\frac{1}{6} \cos 2 \beta \delta M_{Z}^{2}-\frac{2}{3} \cos 2 \beta \delta M_{W}^{2} \\
\delta m_{\tilde{t}_{R}}^{2}= & \left|U_{12}^{\tilde{t}}\right|^{2} \delta m_{\tilde{t}_{1}}^{2}+\left|U_{22}^{\tilde{t}}\right|^{2} \delta m_{\tilde{t}_{2}}^{2}+U_{22}^{\tilde{t}} U_{12}^{\tilde{t} *} \delta Y \\
& +U_{12}^{\tilde{t}} U_{22}^{\tilde{t} *}(\delta Y)^{*}-2 m_{t} \delta m_{t} \\
& +\frac{8}{3} \sin \beta \cos ^{3} \beta M_{Z}^{2} \sin ^{2} \theta_{W} \delta \tan \beta-\frac{2}{3} \cos 2 \beta \delta M_{Z}^{2} \\
& +\frac{2}{3} \cos 2 \beta \delta M_{W}^{2} \\
\delta A_{t}= & \frac{e^{-i \varphi_{u}}}{m_{t}} \\
& \times\left[U_{11}^{\tilde{t}} U_{12}^{\tilde{t} *}\left(\delta m_{\tilde{t}_{1}}^{2}-\delta m_{\tilde{t}_{2}}^{2}\right)+U_{11}^{\tilde{t}} U_{22}^{\tilde{t} *}\left(\delta Y_{\tilde{t}}\right)^{*}\right. \\
& \left.+U_{21}^{\tilde{t}} U_{12}^{\tilde{t} *} \delta Y_{\tilde{t}}-\left(A_{t} e^{i \varphi_{u}}-\frac{\mu_{\mathrm{eff}}^{*}}{\tan \beta}\right) \delta m_{t}\right] \\
& -\frac{e^{-i \varphi_{u}} \mu_{\mathrm{eff}}^{*} \delta \tan \beta}{\tan \beta}+\frac{e^{-i \varphi_{u}} \delta \mu_{\mathrm{eff}}^{*}}{\tan \beta} \\
&
\end{aligned}
$$

where

$$
\begin{aligned}
\delta m_{\tilde{t}_{1}}^{2} & =\operatorname{Re} \Sigma_{11}^{\tilde{t}}\left(m_{\tilde{t}_{1}}^{2}\right) \\
\delta m_{\tilde{t}_{2}}^{2} & =\operatorname{Re} \Sigma_{22}^{\tilde{t}}\left(m_{\tilde{t}_{2}}^{2}\right) \\
\delta Y_{\tilde{t}} & =\left[U^{\tilde{t}} \delta M_{\tilde{t}} U^{\tilde{t} \dagger}\right]_{12}=\left[U^{\tilde{t}} \delta M_{\tilde{t}} U^{\tilde{t} \dagger}\right]_{21}^{*} \\
& =\frac{1}{2} \widetilde{\operatorname{Re}}\left(\Sigma_{12}^{\tilde{t}}\left(m_{\tilde{t}_{1}}^{2}\right)+\Sigma_{12}^{\tilde{t}}\left(m_{\tilde{t}_{2}}^{2}\right)\right) .
\end{aligned}
$$

\footnotetext{
${ }^{14}$ In the Denner description, $\operatorname{Re} \hat{\Sigma}_{i i}^{\tilde{q}}$ is replaced by $\widetilde{\operatorname{Re}} \hat{\Sigma}_{i i}^{\tilde{q}}$ in Eqs. (3.52) and (3.54) while in Eq. (3.53) $\operatorname{Re} \hat{\Sigma}_{i j}^{\tilde{q}}$ is replaced by $\widetilde{\operatorname{Re}} \hat{\Sigma}_{i j}^{\tilde{q}}$.

15 In the Denner description, $\operatorname{Re} \Sigma_{i i}^{\tilde{t}}$ in Eqs. (3.58) and (3.59) is replaced by $\widetilde{\operatorname{Re}} \Sigma_{i i}^{\tilde{t}}$. Note, however, that Eq. (3.60) is the same in both the EMT and the Denner description. We use $\widetilde{R e}$ in the definition of $\delta Y_{\tilde{q}}(q=t, b)$ so that $\delta A_{q}=\left(\delta A_{q}^{*}\right)^{*}$. The contribution from the imaginary part of the loop integrals is then moved into $\delta Z_{i j}^{\tilde{q}}$ and $\delta \bar{Z}_{i j}^{\tilde{q}}$.
}

There remain two parameters from the bottom squark sector to be determined, $A_{b}, m_{\tilde{b}_{R}}^{2}$. We choose the OS scheme where the bottom squark with the dominant contribution from the right-handed sbottom, ${ }^{16}$ which we denote by $\tilde{b}_{i_{R}}$, is OS and the mixing between the two bottom squark states vanishes. The three counterterms $\delta m_{\tilde{b}_{R}}^{2}, \operatorname{Re} \delta A_{b}, \operatorname{Im} \delta A_{b}$ are then obtained by solving three linear equations

$$
\begin{aligned}
& \left|U_{i_{R} 2}^{\tilde{b}}\right|^{2} x+2 m_{b} \operatorname{Re}\left[U_{i_{R} 2}^{\tilde{b}} U_{i_{R} 1}^{\tilde{b} *}\right] y \\
& \quad-2 m_{b} \operatorname{Im}\left[U_{i_{R} 2}^{\tilde{b}} U_{i_{R} 1}^{\tilde{b} *}\right] z=d_{1} \\
& \operatorname{Re}\left[U_{12}^{\tilde{b}} U_{22}^{\tilde{b} *}\right] x+m_{b} \operatorname{Re}\left[U_{12}^{\tilde{b}} U_{21}^{\tilde{b} *}+U_{11}^{\tilde{b}} U_{22}^{\tilde{b} *}\right] y \\
& \quad-m_{b} \operatorname{Im}\left[U_{12}^{\tilde{b}} U_{21}^{\tilde{b} *}-U_{11}^{\tilde{b}} U_{22}^{\tilde{b} *}\right] z=\operatorname{Re} d_{2} \\
& \operatorname{Im}\left[U_{12}^{\tilde{b}} U_{22}^{\tilde{b} *}\right] x+m_{b} \operatorname{Im}\left[U_{12}^{\tilde{b}} U_{21}^{\tilde{b} *}+U_{11}^{\tilde{b}} U_{22}^{\tilde{b} *}\right] y \\
& \quad+m_{b} \operatorname{Re}\left[U_{12}^{\tilde{b}} U_{21}^{\tilde{b} *}-U_{11}^{\tilde{b}} U_{22}^{\tilde{b} *}\right] z=\operatorname{Im} d_{2},
\end{aligned}
$$

where $(x, y, z)=\left(\delta m_{\tilde{b}_{R}}^{2}, \operatorname{Re} \delta A_{b}, \operatorname{Im} \delta A_{b}\right)$ and $^{17}$

$$
\begin{aligned}
d_{1} & =\operatorname{Re} \Sigma_{i_{R} i_{R}}^{\tilde{b}}\left(m_{\tilde{b}_{i_{R}}}^{2}\right)-\left.\left(U^{\tilde{b}} \delta M_{\tilde{b}} U^{\tilde{b} \dagger}\right)_{i_{R}, i_{R}}\right|_{x=y=z=0} \\
d_{2} & =\delta Y_{\tilde{b}}-\left.\left(U^{\tilde{b}} \delta M_{\tilde{b}} U^{\tilde{b} \dagger}\right)_{12}\right|_{x=y=z=0} \\
\delta Y_{\tilde{b}} & =\frac{1}{2} \widetilde{\operatorname{Re}}\left(\Sigma_{12}^{\tilde{b}}\left(m_{\tilde{b}_{1}}^{2}\right)+\Sigma_{12}^{\tilde{b}}\left(m_{\tilde{b}_{2}}^{2}\right)\right)
\end{aligned}
$$

where we have introduced the notation $\delta Y_{\tilde{b}}$ for later use. The other bottom squark mass gets loop corrections. ${ }^{18}$ Its loopcorrected mass $M_{\tilde{b}_{j}}$ is obtained by solving iteratively the following equation ${ }^{19}$

$M_{\tilde{b}_{j}}^{2}=m_{\tilde{b}_{j}}^{2}-\operatorname{Re} \hat{\Sigma}_{j j}^{\tilde{b}}\left(M_{\tilde{b}_{j}}^{2}\right)$

We stop the iteration when the difference between two consecutive solutions is less than $10^{-5}$.

16 We find the right-handed sbottom by looking at the tree-level rotation matrix $U^{\tilde{b}}$. If $\left|U_{12}^{\tilde{b}}\right|$ is larger than $\left|U_{22}^{\tilde{b}}\right|$ then the right-handed sbottom is the $\tilde{b}_{1}$ state, otherwise it is the $\tilde{b}_{2}$ state.

${ }^{17}$ In the Denner description, $\operatorname{Re} \Sigma_{i_{R} i_{R}}^{\tilde{b}}$ in Eq. (3.64) is replaced by $\widetilde{\operatorname{Re}} \Sigma_{i_{R} i_{R}}^{\tilde{b}}$.

18 Note that in principle all four masses of the stops and sbottoms can be renormalized on-shell simultaneously by adapting the input parameters appropriately.

${ }^{19}$ In the Denner description $\operatorname{Re} \hat{\Sigma}_{j j}^{\tilde{b}}$ is replaced by $\widetilde{\operatorname{Re}} \hat{\Sigma}_{j j}^{\tilde{b}}$. 
The OS wave function renormalization constants for the squarks are given by ${ }^{20}$

$$
\begin{aligned}
\delta Z_{i i}^{\tilde{q}} & =-\left.\frac{\partial \operatorname{Re} \Sigma_{i i}^{\tilde{q}}\left(p^{2}\right)}{\partial p^{2}}\right|_{p^{2}=m_{\tilde{q}_{i}}^{2}} \\
\delta Z_{12}^{\tilde{q}} & =2 \frac{\Sigma_{12}^{\tilde{q}}\left(m_{\tilde{q}_{2}}^{2}\right)-\delta Y_{\tilde{q}}}{m_{\tilde{q}_{1}}^{2}-m_{\tilde{q}_{2}}^{2}} \\
\delta Z_{21}^{\tilde{q}} & =-2 \frac{\Sigma_{21}^{\tilde{q}}\left(m_{\tilde{q}_{1}}^{2}\right)-\delta Y_{\tilde{q}}^{*}}{m_{\tilde{q}_{1}}^{2}-m_{\tilde{q}_{2}}^{2}} \\
\delta \bar{Z}_{12}^{\tilde{q}} & =-2 \frac{\Sigma_{12}^{\tilde{q}}\left(m_{\tilde{q}_{1}}^{2}\right)-\delta Y_{\tilde{q}}}{m_{\tilde{q}_{1}}^{2}-m_{\tilde{q}_{2}}^{2}} \\
\delta \bar{Z}_{21}^{\tilde{q}} & =2 \frac{\Sigma_{21}^{\tilde{q}}\left(m_{\tilde{q}_{2}}^{2}\right)-\delta Y_{\tilde{q}}^{*}}{m_{\tilde{q}_{1}}^{2}-m_{\tilde{q}_{2}}^{2}},
\end{aligned}
$$

where the $\delta Y_{\tilde{q}}$ are given in Eqs. (3.60) and (3.66) for $q=t$ and $q=b$, respectively. Besides the OS scheme we also provide the option to use the $\overline{\mathrm{DR}}$ scheme for all parameters and the wave function renormalization constants. In the $\overline{\mathrm{DR}}$ scheme, all squarks receive loop-corrections to their masses. We will discuss this issue in Sect. 3.4.

\subsection{Loop-corrected Higgs boson masses and mixings}

Since we use the mixed OS- $\overline{\mathrm{DR}}$ renormalization scheme for the Higgs sector parameters together with the $\overline{\mathrm{DR}}$ scheme for the Higgs fields, all Higgs bosons are mixed and receive loop corrections to their masses. For the evaluation of the loopcorrected Higgs boson masses and the Higgs mixing matrix, we use the numerical results obtained from NMSSMCALC [14]. In this code the two-loop corrected Higgs boson masses are obtained by determining the zeros of the determinant of the two-point function $\hat{\Gamma}\left(p^{2}\right)$ with

$$
\left(\hat{\Gamma}\left(p^{2}\right)\right)_{i j}=i \delta_{i j}\left(p^{2}-m_{h_{i}}^{2}\right)+i \hat{\Sigma}_{i j}\left(p^{2}\right), i, j=1, \ldots, 5,
$$

where $m_{h_{i}}$ are the tree-level masses and $\hat{\Sigma}_{i j}\left(p^{2}\right)$ is the renormalized self-energy of the $h_{i} \rightarrow h_{j}$ transition at $p^{2}$. In NMSSMCALC, we have included in the renormalized Higgs self-energies the complete one-loop contributions with full momentum dependence $[15,16]$ and the two-loop contributions of $\mathcal{O}\left(\alpha_{t} \alpha_{s}\right)$ [12] and of $\mathcal{O}\left(\alpha_{t}^{2}\right)$ [18] in the gaugeless limit at zero momentum. We note that the renormalized oneloop Higgs boson self-energies have been computed in the

$\overline{20}$ In the Denner description, $\operatorname{Re} \Sigma_{i i}^{\tilde{q}}$ in Eq. (3.68) is replaced by $\widetilde{\operatorname{Re}} \Sigma_{i i}^{\tilde{q}}$ and $\operatorname{Re} \Sigma_{12}^{\tilde{q}}, \operatorname{Re} \Sigma_{21}^{\tilde{q}}$ in Eqs. (3.69)-(3.72) are replaced by $\widetilde{\operatorname{Re}} \Sigma_{12}^{\tilde{q}}, \widetilde{\operatorname{Re}} \Sigma_{21}^{\tilde{q}}$, respectively.
'tHooft-Feynman gauge. The self-energies mixing the treelevel neutral Higgs bosons and the Goldstone boson or the longitudinal component of the $Z$ boson, denoted by $\hat{\Sigma}_{h_{i} G / Z}$, give negligible contributions to the loop-corrected Higgs boson masses, as we confirmed numerically. We therefore do not include $\hat{\Sigma}_{h_{i} G / Z}$ in the two-point function defined in Eq. (3.73). We take the mixings into account, however, in the decays of the neutral Higgs bosons. This will be specified in the sections presenting the computation of the decay widths. The loop-corrected masses of the Higgs bosons are then sorted by ascending masses ${ }^{21}$

$M_{H_{1}} \leq M_{H_{2}} \leq M_{H_{3}} \leq M_{H_{4}} \leq M_{H_{5}}$.

We have improved the stability of the determination of the Higgs boson masses in NMSSMCALC by implementing twopoint loop integrals with complex momentum. In the old version of NMS SMCALC, in order to take into account the contribution of the imaginary part of the complex momentum we expanded the renormalized Higgs self-energies around the real part of the complex momentum as

$\hat{\Sigma}_{i j}\left(p^{2}\right)=\hat{\Sigma}_{i j}\left(\operatorname{Re} p^{2}\right)+i \operatorname{Im} p^{2} \frac{\partial \hat{\Sigma}_{i j}\left(\operatorname{Re} p^{2}\right)}{\partial \operatorname{Re} p^{2}}$.

Note that this was done only for the one-loop correction with full momentum dependence. This expansion is not good when $\operatorname{Re} p^{2}$ is close to threshold regions in which $\partial \hat{\Sigma}_{i j}\left(\operatorname{Re} p^{2}\right) / \partial \operatorname{Re} p^{2}$ contains threshold singularities. To overcome this problem one can use complex masses for the loop particles or complex momenta. Using complex masses requires the decay widths of the particles. These have to be obtained in an iterative procedure which is very time consuming. We have decided to use the complex momenta and to keep the masses real. We have implemented the two-point loop integrals with complex momenta and therefore do not use any more the mentioned approximation. We have confirmed that the evaluation of the Higgs masses is stable in the singularities region and the differences between Higgs masses using the complex momentum and the expansion in Eq. $((3.75))$, defined as $\left(M_{H_{i}}^{\text {expansion }}-\right.$ $\left.M_{H_{i}}^{\text {complex } p^{2}}\right) / M_{H_{i}}^{\text {expansion }}(i=1, \ldots, 5)$ are of per mille level.

The renormalized Higgs boson self-energies $\hat{\Sigma}_{i j}$ can be decomposed into one-loop and two-loop contributions denoted by the superscript (1) and (2), respectively,

$\hat{\Sigma}_{i j}=\hat{\Sigma}_{i j}^{(1)}\left(p^{2}\right)+\hat{\Sigma}_{i j}^{(2)}(0)$.

The complete one-loop corrections to the neutral NMSSM Higgs bosons have been computed in Refs. [15,16] for the $\mathrm{CP}$-conserving and $\mathrm{CP}$-violating NMSSM, respectively.

${ }^{21}$ We denote loop-corrected Higgs mass eigenstates by capital letters $H_{i}(i=1, \ldots, 5)$. 
The renormalized two-loop self-energies $\hat{\Sigma}_{i j}(0)$ are evaluated in the approximation of vanishing external momentum, i.e. $p^{2}=0$, and include the $\mathcal{O}\left(\alpha_{t} \alpha_{s}\right)$ corrections calculated in [17] and the $\mathcal{O}\left(\alpha_{t}^{2}\right)$ contributions of [18]. Hence,

$\hat{\Sigma}^{(2)}(0)_{i j}=\hat{\Sigma}_{i j}^{(2), \alpha_{t} \alpha_{s}}(0)+\hat{\Sigma}_{i j}^{(2), \alpha_{t}^{2}}(0)$.

The renormalized one-loop Higgs self-energy for the transition $h_{i} \rightarrow h_{j}(i, j=1, \ldots, 5)$ is given by

$$
\begin{aligned}
\hat{\Sigma}_{i j}^{\oplus}\left(p^{2}\right)= & \Sigma_{i j}^{\oplus}\left(p^{2}\right)+\frac{1}{2} p^{2}\left[\mathcal{R}\left(\delta^{\oplus} \mathcal{Z}^{\dagger}+\delta^{\oplus} \mathcal{Z}\right) \mathcal{R}^{T}\right]_{i j} \\
& -\left[\mathcal{R}\left(\frac{1}{2} \delta^{\oplus} \mathcal{Z}^{\dagger} \mathcal{M}_{h h}+\frac{1}{2} \mathcal{M}_{h h} \delta^{\oplus} \mathcal{Z}+\delta^{\oplus} \mathcal{M}_{h h}\right) \mathcal{R}^{T}\right]_{i j} .
\end{aligned}
$$

The terms in the square brackets represent the one-loop counterterm consisting of the wave function renormalization constant matrix $\delta^{\oplus} \mathcal{Z}$ and the mass counterterm $\delta^{\oplus} \mathcal{M}_{h h}$. The renormalized self-energy at two-loop order is given by

$$
\begin{aligned}
\hat{\Sigma}_{i j}^{\ominus}\left(p^{2}\right)= & \Sigma_{i j}^{\ominus}\left(p^{2}\right) \\
& +\frac{1}{2} p^{2}\left[\mathcal{R}\left(\frac{1}{2}\left(\delta^{\oplus} \mathcal{Z}\right)^{\dagger} \delta^{0} \mathcal{Z}+\delta^{\theta} \mathcal{Z}^{\dagger}+\delta^{\theta} \mathcal{Z}\right) \mathcal{R}^{T}\right]_{i j} \\
& -\left(\delta^{\ominus} M^{2}\right)_{i j},
\end{aligned}
$$

where $\Sigma_{i j}^{(2)}\left(p^{2}\right)$ is the unrenormalized two-loop self-energy evaluated at $p^{2}=0$ and $\left(\delta^{\ominus} M^{2}\right)_{i j}$ denotes the two-loop mass counterterm,

$$
\begin{aligned}
\left(\delta^{\oplus} M^{2}\right)_{i j}= & \frac{1}{2}\left[\mathcal { R } \left(\frac{1}{2}\left(\delta^{\oplus} \mathcal{Z}\right)^{\dagger} \mathcal{M}_{h h} \delta^{\oplus} \mathcal{Z}+\delta^{\oplus} \mathcal{Z}^{\dagger} \delta^{\oplus} \mathcal{M}_{h h}\right.\right. \\
& \left.\left.+\delta^{\oplus} \mathcal{M}_{h h} \delta^{\oplus} \mathcal{Z}+\delta^{\oplus} \mathcal{Z}^{\dagger} \mathcal{M}_{h h}+\mathcal{M}_{h h} \delta^{\oplus} \mathcal{Z}\right) \mathcal{R}^{T}\right]_{i j} \\
& +\left(\mathcal{R} \delta^{\oplus} \mathcal{M}_{h h} \mathcal{R}^{T}\right)_{i j} .
\end{aligned}
$$

The Higgs field renormalization constant matrix is diagonal and reads

$$
\begin{aligned}
\delta^{(n)} \mathcal{Z}= & \operatorname{diag}\left(\Delta^{(n)} Z_{H_{d}}, \Delta^{(n)} Z_{H_{u}}, \Delta^{(n)} Z_{S}, s_{\beta}^{2} \Delta^{(n)} Z_{H_{d}}\right. \\
& \left.+c_{\beta}^{2} \delta^{(n)} Z_{H_{u}}, \Delta^{(n)} Z_{S}\right), \quad n=1,2,
\end{aligned}
$$

with the renormalization constants $\Delta^{(n)} Z_{H_{u}, H_{d}, S}$ for the doublet and singlet fields. In terms of the Higgs wave function renormalization constants $\delta Z_{i}^{(1,2)}\left(i=H_{u}, H_{d}, H_{S}\right)$ at oneand two-loop order, respectively, they read for $n=1$

$\Delta^{(1)} Z_{i} \equiv \delta^{(1)} Z_{i}$

and for $n=2$,

$\Delta^{(2)} \equiv \delta^{(2)} Z_{i}-\frac{1}{4}\left(\delta^{(1)} Z_{i}\right)^{2}$.

We point out that in the above formulae the momentum dependence is kept for the purpose of defining the Higgs field renormalization constants, while the computations at two-loop level are perfomed in the approximation of vanishing external momentum, i.e. for $p^{2}=0$. The mass matrix counterterms $\delta \mathcal{M}_{h h}^{(1,2)}$ implicitly contain the counterterms of the parameters that need to be renormalized. The renormalization of these parameters and of the Higgs fields has been presented in Sect. 3.1.1. For further details on the computation of the one- and two-loop corrections to the Higgs boson masses, we refer to [15-18].

In processes with external Higgs bosons finite wavefunction renormalization factors $\mathbf{Z}^{H}$ have to be taken into account in order to ensure the on-shell properties of these Higgs bosons. The wave-function renormalization factor matrix performing the rotation to the OS states is given by [30]

$$
\mathbf{Z}^{H}=\left(\begin{array}{cccc}
\sqrt{\hat{Z}_{h_{1}}} & \sqrt{\hat{Z}_{h_{1}}} \hat{Z}_{h_{1} h_{2}} \sqrt{\hat{Z}_{h_{1}}} \hat{Z}_{h_{1} h_{3}} \sqrt{\hat{Z}_{h_{1}}} \hat{Z}_{h_{1} h_{4}} \sqrt{\hat{Z}_{h_{1}}} \hat{Z}_{h_{1} h_{5}} \\
\sqrt{\hat{Z}_{h_{2}}} \hat{Z}_{h_{2} h_{1}} & \sqrt{\hat{Z}_{h_{2}}} & \sqrt{\hat{Z}_{h_{2}}} \hat{Z}_{h_{2} h_{3}} \sqrt{\hat{Z}_{h_{2}}} \hat{Z}_{h_{2} h_{4}} \sqrt{\hat{Z}_{h_{2}}} \hat{Z}_{h_{2} h_{5}} \\
\sqrt{\hat{Z}_{h_{3}}} \hat{Z}_{h_{3} h_{1}} \sqrt{\hat{Z}_{h_{3}}} \hat{Z}_{h_{3} h_{2}} & \sqrt{\hat{Z}_{h_{3}}} & \sqrt{\hat{Z}_{h_{3}}} \hat{Z}_{h_{3} h_{4}} & \sqrt{\hat{Z}_{h_{3}}} \hat{Z}_{h_{3} h_{5}} \\
\sqrt{\hat{Z}_{h_{4}}} \hat{Z}_{h_{4} h_{1}} \sqrt{\hat{Z}_{h_{4}}} \hat{Z}_{h_{4} h_{2}} \sqrt{\hat{Z}_{h_{4}}} \hat{Z}_{h_{4} h_{3}} & \sqrt{\hat{Z}_{h_{4}}} & \sqrt{\hat{Z}_{h_{4}}} \hat{Z}_{h_{4} h_{5}} \\
\sqrt{\hat{Z}_{h_{5}}} \hat{Z}_{h_{5} h_{1}} \sqrt{\hat{Z}_{h_{5}}} \hat{Z}_{h_{5} h_{2}} \sqrt{\hat{Z}_{h_{5}}} \hat{Z}_{h_{5} h_{3}} \sqrt{\hat{Z}_{h_{5}}} \hat{Z}_{h_{5} h_{4}} & \sqrt{\hat{Z}_{h_{5}}}
\end{array}\right)
$$

where

$\hat{Z}_{h_{i}}=\frac{1}{\left(\frac{i}{\Delta_{i i}^{h}\left(p^{2}\right)}\right)^{\prime}\left(M_{H_{i}}^{2}\right)}, \quad \hat{Z}_{h_{i} h_{j}}=\left.\frac{\Delta_{i j}^{h}\left(p^{2}\right)}{\Delta_{i i}^{h}\left(p^{2}\right)}\right|_{p^{2}=M_{H_{i}}^{2}}$,

with

$\Delta^{h}=-\left[\hat{\Gamma}^{h}\left(p^{2}\right)\right]^{-1}$.

Here prime denotes the derivative with respect to $p^{2}$.

\subsection{Loop-corrected neutralino and chargino masses}

Within the OS and $\overline{\mathrm{DR}}$ schemes defined for the neutralino and chargino sector, the electroweakinos cannot all be renormalized OS, and there remain neutralinos and charginos that receive loop corrections to their masses. In the following we define our procedure to determine the loop-corrected masses for fermions in the general case where mixing contributions are present. ${ }^{22}$ This procedure will be used for both the $\mathrm{OS}$ and the $\overline{\mathrm{DR}}$ scheme. To give an intuitive definition of

\footnotetext{
${ }^{22}$ In the literature there exist many papers that deal with loop-corrected masses for neutralinos and charginos in the MSSM such as Refs. $[26,31,32]$ to name a few of them. For the definition of the loopcorrected masses applied there, OS conditions for the field renormalization constants were applied to eliminate mixing effects between fermions at tree-level mass values.
} 
the loop-corrected fermion masses, we express the propagator matrix in terms of left- and right-handed Weyl spinors, $\psi_{D}=\left(\psi_{L}, \psi_{R}\right)^{T}$. In this basis, the tree-level propagator matrix is given by

$S(p)=-\Gamma(p)^{-1}, \quad \Gamma(p)=i\left(\begin{array}{cc}p^{\mu} \sigma_{\mu} & -m \\ -m & p^{\mu} \bar{\sigma}_{\mu}\end{array}\right)$,

with

$\sigma^{\mu}=(\mathbb{1}, \boldsymbol{\sigma}) \quad$ and $\quad \bar{\sigma}^{\mu}=(\mathbb{1},-\boldsymbol{\sigma})$,

where $\sigma=\left(\sigma^{1}, \sigma^{2}, \sigma^{3}\right)$ denotes the three Pauli matrices. The mass matrix is given by

$$
\left(\begin{array}{cc}
0 & m \\
m & 0
\end{array}\right) \text {. }
$$

The loop-corrected propagator matrix in the Weyl basis $\left(\psi_{L}, \psi_{R}\right)^{T}$ is defined as

$S(p)=i\left(\begin{array}{cc}p^{\mu} \sigma_{\mu}\left(1+\hat{\Sigma}^{L}\left(p^{2}\right)\right) & -m+\hat{\Sigma}^{L s}\left(p^{2}\right) \\ -m+\hat{\Sigma}^{R s}\left(p^{2}\right) & p^{\mu} \bar{\sigma}_{\mu}\left(1+\hat{\Sigma}^{R}\left(p^{2}\right)\right)\end{array}\right)^{-1}$.

The loop-corrected mass $M$ is determined from the real pole $p^{2}=M^{2}$ of the propagator matrix satisfying the equation

$\operatorname{det}\left(\begin{array}{cc}p^{\mu} \sigma_{\mu}\left(1+\operatorname{Re} \hat{\Sigma}^{L}\left(p^{2}\right)\right) & -m+\operatorname{Re} \hat{\Sigma}^{L s}\left(p^{2}\right) \\ -m+\operatorname{Re} \hat{\Sigma}^{R s}\left(p^{2}\right) & p^{\mu} \bar{\sigma}_{\mu}\left(1+\operatorname{Re} \hat{\Sigma}^{R}\left(p^{2}\right)\right)\end{array}\right)=0$.

The solution of Eq. (3.91) is given by

$p^{2}=\frac{m^{2}\left(1-\operatorname{Re} \hat{\Sigma}^{L s}\left(p^{2}\right) / m\right)\left(1-\operatorname{Re} \hat{\Sigma}^{R s}\left(p^{2}\right) / m\right)}{\left(1+\operatorname{Re} \hat{\Sigma}^{L}\left(p^{2}\right)\right)\left(1+\operatorname{Re} \hat{\Sigma}^{R}\left(p^{2}\right)\right)}$,

which can be solved iteratively. When the fermion is OS, $p^{2}=m^{2}$, the above relation is nothing else but the OS condition obtained from Eq. (3.21). For the case of $n$ Dirac spinors, the 1PI two-point function in the basis $\left(\psi_{L}^{1}, \psi_{L}^{2}, \ldots, \psi_{L}^{n}, \psi_{R}^{1}, \psi_{R}^{2}, \ldots, \psi_{R}^{n}\right)$ is a $2 n \times 2 n$ matrix

$\Gamma(p)=i\left(\begin{array}{cc}p^{\mu} \sigma_{\mu} a\left(p^{2}\right) & d\left(p^{2}\right) \\ c\left(p^{2}\right) & p^{\mu} \bar{\sigma}_{\mu} b\left(p^{2}\right)\end{array}\right)$.

The matrices $a, b, c, d$ are $2 \times 2$ matrices (i.e. $n=2$ ) in case of charginos and $5 \times 5$ matrices (i.e. $n=5$ ) for neutralinos. With $m_{\tilde{x}_{i}}$ generically denoting the mass of an electroweakino with index $i$, the matrices are given by

$$
\begin{aligned}
& a_{i j}\left(p^{2}\right)=\delta_{i j}+\hat{\Sigma}_{i j}^{L}\left(p^{2}\right) \\
& b_{i j}\left(p^{2}\right)=\delta_{i j}+\hat{\Sigma}_{i j}^{R}\left(p^{2}\right) \\
& c_{i j}\left(p^{2}\right)=-m_{\tilde{\chi}_{i}} \delta_{i j}+\hat{\Sigma}_{i j}^{L s}\left(p^{2}\right) \\
& d_{i j}\left(p^{2}\right)=-m_{\tilde{\chi}_{i}} \delta_{i j}+\hat{\Sigma}_{i j}^{R s}\left(p^{2}\right),
\end{aligned}
$$

with $i, j=1,2$ for charginos and $i, j=1, \ldots, 5$ for neutralinos.

The poles of the propagator matrix are the solutions of the equation

$\operatorname{det}[\Gamma(p)]=0$.

This is equivalent to [33],

$$
\operatorname{det}\left[p^{2}-c\left(p^{2}\right) b^{-1}\left(p^{2}\right) d\left(p^{2}\right) a^{-1}\left(p^{2}\right)\right]=0 .
$$

In practice, we solve the equation numerically through iteration together with the diagonalization of the mass matrix $M_{\tilde{\chi}}=c\left(p^{2}\right) b^{-1}\left(p^{2}\right) d\left(p^{2}\right) a^{-1}\left(p^{2}\right)$ to obtain the complex poles. The loop-corrected masses are then obtained from the real parts of these complex poles. This procedure is applied for the calculation of the loop-corrected masses for neutralinos using the OS definition of the neutralino WFR constants. However, for the chargino sector the mass matrix $M_{\tilde{\chi}^{ \pm}}$contains infrared (IR) divergences at arbitrary momentum. We have implemented the approximation used in Ref. [26] and calculate the loop-corrected chargino masses by using the formula

$$
\begin{aligned}
M_{\tilde{\chi}_{i}^{+}}= & m_{\tilde{\chi}_{i}^{+}}\left(1-\frac{1}{2} \operatorname{Re} \Sigma_{i i}^{\tilde{\chi}_{i}^{+}, L}\left(m_{\tilde{\chi}_{i}^{+}}^{2}\right)-\frac{1}{2} \operatorname{Re} \Sigma_{i i}^{\tilde{\chi}_{i}^{+}, R}\left(m_{\tilde{\chi}_{i}^{+}}^{2}\right)\right) \\
& -\frac{1}{2} \operatorname{Re} \Sigma_{i i}^{\tilde{\chi}_{i}^{+}, L s}\left(m_{\tilde{\chi}_{i}^{+}}^{2}\right) \\
& -\frac{1}{2} \operatorname{Re} \Sigma_{i i}^{\tilde{\chi}_{i}^{+}, R s}\left(m_{\tilde{\chi}_{i}^{+}}^{2}\right)+\operatorname{Re}\left(U^{*} \delta M_{C} V^{\dagger}\right)_{i i} .
\end{aligned}
$$

\subsection{Loop-corrected squark masses and mixings}

In our $\overline{\mathrm{DR}}$ scheme, the counterterms

$\delta m_{t}, \delta m_{b}, \delta m_{\tilde{Q}_{3}}^{2}, \delta m_{\tilde{t}_{R}}^{2}, \delta m_{\tilde{b}_{R}}^{2}, \delta A_{t}, \delta A_{b}$

contain only the UV divergent parts. For the renormalization of the squark fields we use a modified OS scheme. In the following, we will describe the details of this scheme. We first compute the $\overline{\mathrm{DR}}$ squark WFR constants by taking the UVdivergent parts of the OS counterterms, defined in Eqs. (3.68) to (3.72), with tree-level mass values. Using these $\overline{\mathrm{DR}}$ squark WFR constants, we then compute the loop-corrected squark masses $M_{\tilde{q}_{i}}$ that are obtained by solving iteratively the equations

$M_{\tilde{q}_{i}}^{2}=m_{\tilde{q}_{i}}^{2}-\operatorname{Re} \hat{\Sigma}_{i i}^{\tilde{q}}\left(M_{\tilde{q}_{i}}^{2}\right), \quad i=1,2$.

We have assumed here that the off-diagonal renormalized self-energies vanish and that the residues of the propagators are unity at the loop-corrected masses. This is equivalent to redefining the diagonal squark WFR constants at loop- 
corrected masses as

$$
\begin{aligned}
& \delta Z_{i i}^{\tilde{q}}\left(M_{\tilde{q}_{i}}^{2}\right)=-\left.\frac{\partial \Sigma_{i i}^{\tilde{q} \mathrm{div}}\left(p^{2}\right)}{\partial p^{2}}\right|_{p^{2}=m_{\tilde{q}_{i}}^{2}}-\left.\frac{\partial \hat{\Sigma}_{i i}^{\tilde{q}}\left(p^{2}\right)}{\partial p^{2}}\right|_{p^{2}=M_{\tilde{q}_{i}}^{2}}, \\
& i=1,2,
\end{aligned}
$$

and the off-diagonal WFR constants as

$$
\begin{aligned}
& \delta Z_{i j}^{\tilde{q}}\left(M_{\tilde{q}_{k}}^{2}\right)=\frac{\Sigma_{i j}^{\tilde{q} \text { div }}\left(m_{\tilde{q}_{i}}^{2}\right)-\Sigma_{i j}^{\tilde{q} \text { div }}\left(m_{\tilde{q}_{j}}^{2}\right)}{m_{\tilde{q}_{j}}^{2}-m_{\tilde{q}_{i}}^{2}}-2 \frac{\hat{\Sigma}_{i j}^{\tilde{q}}\left(M_{\tilde{q}_{k}}^{2}\right)}{M_{\tilde{q}_{k}}^{2}-m_{\tilde{q}_{i}}^{2}}, \\
& i, j, k=1,2, \quad i \neq j .
\end{aligned}
$$

In the above equations the renormalized self-energies are computed with the $\overline{\mathrm{DR}}$ squark WFR constants. We keep also the imaginary part of the two-point loop integrals in the renormalized self-energies. Note that the WFR constants $\delta Z_{i j}^{\tilde{q}}\left(M_{\tilde{q}_{k}}^{2}\right)$ will enter the evaluation of the decay width. The diagonal WFR constants $\delta Z_{i i}^{\tilde{q}}\left(M_{\tilde{q}_{k}}^{2}\right)$ contain IR divergences evaluated at the loop-corrected masses. These IR divergences will cancel exactly with those arising from the virtual part and the real radiation contributions which are also evaluated at the loop-corrected masses. We have verified that this statement is true for both the EW and the QCD corrections.

\section{Higher-order corrections to the two-body decays of the neutral Higgs bosons}

In this section, we present those two-body decay channels that we have improved by including the missing NLO EW and QCD corrections. These channels are the decays into OS SM fermion pairs, OS massive gauge bosons, into a pair of Higgs and gauge bosons, into chargino or neutralino pairs and into top or bottom squark pairs. We will not discuss decays into gluon pairs, photon pairs or $Z \gamma$ which can be found in Ref. [14]. For these decays, NLO EW corrections are of twoloop order as the leading order (LO) decay widths are already loop-induced. The inclusion of the NLO EW corrections to Higgs-to-Higgs decays on the other hand have been presented in Ref. [11] and the dominant two-loop corrections of the $\mathcal{O}\left(\alpha_{t} \alpha_{s}\right)$ have been provided in Ref. [12].

For our computation we have used several programs. The generation of the amplitudes was done by FeynArts [34, 35] using a model file created by SARAH [36-39]. The output amplitudes were further processed using FeynCalc [40,41] for the simplification of the Dirac matrices and for the tensor reduction. The one-loop integrals were evaluated with the help of LoopTools [42].

\subsection{Higgs boson decays into fermion pairs}

In order to make use of the published results of higher-order corrections in the literature for $\mathrm{CP}$-even and $\mathrm{CP}$-odd Higgs bosons, it is convenient to write the interaction vertex of the complex NMSSM Higgs boson $h_{i}(i=1, \ldots, 5)$ and quarks as

$\mathcal{L}_{h_{i} q \bar{q}}=-\frac{m_{q}}{v} \bar{q} h_{i}\left(g_{h_{i} q \bar{q}}^{S}-i g_{h_{i} q \bar{q}}^{P} \gamma_{5}\right) q$,

where the scalar and pseudoscalar coupling coefficients for the up- and down-type quarks at tree-level are given by

$g_{h_{i} d \bar{d}}^{S}=\frac{\mathcal{R}_{i 1}}{c_{\beta}}, \quad g_{h_{i} d \bar{d}}^{P}=\mathcal{R}_{i 4} t_{\beta}$,

$g_{h_{i} u \bar{u}}^{S}=\frac{\mathcal{R}_{i 2}}{s_{\beta}}, \quad g_{h_{i} u \bar{u}}^{P}=\frac{\mathcal{R}_{i 4}}{t_{\beta}}$,

where $\mathcal{R}_{i j}(i, j=1,5)$ denotes the matrix elements of the mixing matrix rotating the tree-level Higgs gauge eigenstates to the mass eigenstates, see Eq. (2.8).

Following the prescription outlined in our publication [14], we improve the widths of the Higgs boson decays into quark pairs by including the missing SUSY-QCD and SUSY-EW corrections. We decompose the EW corrections into the known QED corrections arising from a virtual photon exchange and a real photon emission and the remaining EW corrections from the genuine EW one-loop diagrams.

The one-loop SUSY-QCD corrections originate from loop diagrams with the exchange of a gluino, while the SUSYEW corrections stem from loop diagrams with weak gauge bosons $W, Z$, fermions, Higgs bosons and their superpartners in the internal lines. They are both IR finite quantities. The computation of the Higgs boson decays into a bottom quark pair shows that the bottom quark mass counterterm contains terms proportional to $t_{\beta}$. This contribution is large in the large- $t_{\beta}$ regime and universal. In many cases, this contribution is the leading part of the SUSY-QCD and SUSYEW corrections and can be absorbed into an effective bottom quark Yukawa coupling. This can be done by using an effective Lagrangian formalism [43-45]. In Ref. [14], we have presented the effective bottom Yukawa couplings in the real and complex NMSSM. We do not repeat every detail here but only quote the relevant formulae. In Eq. (4.2) we have given the tree-level scalar and pseudoscalar coupling coefficients appearing in the Feynman rule for the CP-violating Higgs bosons $h_{i}$ to a bottom-quark pair. The Feynman rule for the effective coupling including the leading SUSY-QCD and SUSY-EW corrections [43-52] (denoted by a tilde to mark the inclusion of the corrections) is also decomposed into a scalar and a pseudoscalar part and reads [14]

$$
-\frac{i m_{b}}{v}\left[\tilde{g}_{h_{i} b \bar{b}}^{S}-i \gamma_{5} \tilde{g}_{h_{i} b \bar{b}}^{P}\right],
$$


with

$\tilde{g}_{h_{i} b \bar{b}}^{S}=\operatorname{Re} \tilde{g}_{b_{L}}^{h_{i}} \quad$ and $\quad \tilde{g}_{h_{i} b \bar{b}}^{P}=\operatorname{Im} \tilde{g}_{b_{L}}^{h_{i}}$,

where

$$
\begin{aligned}
\tilde{g}_{b_{L}}^{h_{i}}= & \frac{1}{\left(1+\Delta_{b}\right)}\left[\frac{\mathcal{R}_{i 1}}{\cos \beta}+\frac{\mathcal{R}_{i 2}}{\sin \beta} \Delta_{b}+\frac{\mathcal{R}_{i 3} v}{v_{s}} \Delta_{b}+i \mathcal{R}_{i 4} \tan \beta\right. \\
& \left.\times\left(1-\frac{\Delta_{b}}{\tan ^{2} \beta}\right)-i \frac{\mathcal{R}_{i 5} v}{v_{s}} \Delta_{b}\right] .
\end{aligned}
$$

The correction $\Delta_{b}$ including the leading SUSY-QCD and SUSY-EW corrections can be cast into the form

$\Delta_{b}=\frac{\Delta_{b}^{\mathrm{QCD}}+\Delta_{b}^{\mathrm{elw}}}{1+\Delta_{1}}$

with the one-loop corrections given by

$$
\begin{aligned}
\Delta_{b}^{\mathrm{QCD}} & =\frac{C_{F}}{2} \frac{\alpha_{s}\left(\mu_{R}\right)}{\pi} M_{3}^{*} \mu_{\mathrm{eff}}^{*} \tan \beta I\left(m_{\tilde{b}_{1}}^{2}, m_{\tilde{b}_{2}}^{2}, m_{\tilde{g}}^{2}\right), \\
\Delta_{b}^{\mathrm{elw}} & =\frac{\alpha_{t}\left(\mu_{R}\right)}{4 \pi} A_{t}^{*} \mu_{\mathrm{eff}}^{*} \tan \beta I\left(m_{\tilde{t}_{1}}^{2}, m_{\tilde{t}_{2}}^{2},\left|\mu_{\mathrm{eff}}\right|^{2}\right), \\
\Delta_{1} & =-\frac{C_{F}}{2} \frac{\alpha_{s}\left(\mu_{R}\right)}{\pi} M_{3}^{*} A_{b} I\left(m_{\tilde{b}_{1}}^{2}, m_{\tilde{b}_{2}}^{2}, m_{\tilde{g}}^{2}\right),
\end{aligned}
$$

where $\alpha_{t}=y_{t}^{2} /(4 \pi)$ with $y_{t}=\sqrt{2} m_{t} /(v \sin \beta)$ is the topYukawa coupling and $C_{F}=4 / 3$. The generic function $I$ is defined as

$I(a, b, c)=\frac{a b \log \frac{a}{b}+b c \log \frac{b}{c}+c a \log \frac{c}{a}}{(a-b)(b-c)(a-c)}$.

Note that the scale of $\alpha_{s}$ in the SUSY-QCD corrections has been set equal to $\mu_{R}=\left(m_{\tilde{b}_{1}}+m_{\tilde{b}_{2}}+\left|M_{\tilde{g}}\right|\right) / 3$, while in the SUSY-EW corrections it is $\mu_{R}=\left(m_{\tilde{t}_{1}}+m_{\tilde{t}_{2}}+|\mu|\right) / 3$. The strong coupling constant $\alpha_{s}$ is evaluated with five active flavors.

The decay width of the CP-violating NMSSM Higgs bosons $H_{i}$ into $q \bar{q}$, including the QCD, SUSY-QCD, QED, EW and SUSY-EW corrections, can then be cast into the form

$$
\begin{aligned}
\Gamma\left(H_{i} \rightarrow q \bar{q}\right)= & \frac{3 G_{F} M_{H_{i}}}{4 \sqrt{2} \pi} \bar{m}_{q}^{2}\left(M_{H_{i}}\right) \\
& \times\left[\left(1-4 x_{q}\right)^{3 / 2} \Delta_{\mathrm{QCD}}^{S} \Delta_{\mathrm{QED}}^{S} \Gamma_{H_{i} \rightarrow q \bar{q}}^{S}\right. \\
& \left.+\left(1-4 x_{q}\right)^{1 / 2} \Delta_{\mathrm{QCD}}^{P} \Delta_{\mathrm{QED}}^{P} \Gamma_{H_{i} \rightarrow q \bar{q}}^{P}\right],
\end{aligned}
$$

where $x_{q}=m_{q}^{2} / M_{H_{i}}^{2}$, and

$$
\begin{aligned}
\Gamma_{H_{i} \rightarrow q \bar{q}}^{S}= & \left(\sum_{j=1}^{5} \mathbf{Z}_{i j}^{H} \tilde{g}_{h_{j} q \bar{q}}^{S}\right)\left(\sum_{k=1}^{5} \mathbf{Z}_{i k}^{H} \tilde{g}_{h_{k} q \bar{q}}^{S}\right)^{*} \\
& +2 \operatorname{Re}\left[\left(\sum_{j=1}^{5} \mathbf{Z}_{i j}^{H} \tilde{g}_{h_{j} q \bar{q}}^{S}\right)\left(\sum_{k=1}^{5} \mathbf{Z}_{i k}^{H} \delta \mathcal{M}^{\mathrm{rem}, S}\left(h_{k} \rightarrow q \bar{q}\right)\right)^{*}\right] \\
& +2 \operatorname{Re}\left[\left(\sum_{j=1}^{5} \mathbf{Z}_{i j}^{H} \tilde{g}_{h_{j} q \bar{q}}^{S}\right)\left(\sum_{k=1}^{5} \mathbf{Z}_{i k}^{H} \delta_{\text {sub }}^{S}\left(h_{k} \rightarrow q \bar{q}\right)\right)^{*}\right]
\end{aligned}
$$

and

$$
\begin{aligned}
\Gamma_{H_{i} \rightarrow q \bar{q}}^{P}= & \left.\left(\sum_{j=1}^{5} \mathbf{Z}_{i j}^{H} \tilde{g}_{h_{j} q \bar{q}}^{P}\right)\left(\sum_{k=1}^{5} \mathbf{Z}_{i k}^{H} \tilde{g}_{h_{k}}^{P}\right)^{*}\right)^{*} \\
& +2 \operatorname{Re}\left[\left(\sum_{j=1}^{5} \mathbf{Z}_{i j}^{H} \tilde{g}_{h_{j} q \bar{q}}^{P}\right) \sum_{k=1}^{5}\left(\mathbf{Z}_{i k}^{H}\right)^{*}\right. \\
& \left.\times\left(\delta \mathcal{M}^{\mathrm{rem}, P}\left(h_{k} \rightarrow q \bar{q}\right)+\delta \mathcal{M}_{G Z, \text { mix }}\left(h_{k} \rightarrow q \bar{q}\right)\right)^{*}\right] \\
& +2 \operatorname{Re}\left[\left(\sum_{j=1}^{5} \mathbf{Z}_{i j}^{H} \tilde{g}_{h_{j} q \bar{q}}^{P}\right)\left(\sum_{k=1}^{5} \mathbf{Z}_{i k}^{H} \delta_{\text {sub }}^{P}\left(h_{k} \rightarrow q \bar{q}\right)\right)^{*}\right] .
\end{aligned}
$$

In the numerical analysis presented in Sect. 5 we will use the quantity $\Gamma^{\mathrm{SEW}(+\mathrm{QCD})}$ for the decays into fermion pairs to denote the partial decay widths including the SUSY-EW and (for the quarks) SUSY-QCD corrections, i.e. exactly the partial decay width as defined in Eq. (4.12) with the loopcorrected $\Gamma^{S}$ and $\Gamma^{P}$ given in Eqs. (4.13) and (4.14), respectively. In contrast, we will denote by $\Gamma^{\text {tree }}$ the partial decay widths that only include the $\Delta_{b}$ corrections, i.e. Eq. (4.12) but with $\Gamma^{S}$ and $\Gamma^{P}$ given by the first lines in Eqs. (4.13) and (4.14), respectively.

Note that in $\delta \mathcal{M}^{\text {rem, } S / P}, \delta_{\text {sub }}^{S / P}$ and $\delta \mathcal{M}_{G Z \text {,mix }}$ (which will be explained below) we use the tree-level Higgs couplings $g_{h_{k} q \bar{q}}$ to the quarks. We take the occasion to remind the reader that tree-level mass eigenstates are always denoted by $h_{i}$ and loop-corrected ones by $H_{i}$. Unless stated otherwise, this means that we use tree-level couplings for external $h_{i}$ but with loop-corrected masses, and for particles inside loop diagrams we always use tree-level masses and tree-level couplings. We comment on the various terms appearing in Eqs. (4.12), (4.13) and (4.14) one by one. The one-loop QED corrections, denoted by $\Delta_{\text {QED }}$ have been known in the SM for a long time, $c f$. Refs. [53-58]. In the limit $m_{q} \ll M_{H_{i}}$ they are given by Ref. [59], ${ }^{23}$

\footnotetext{
${ }^{23}$ Note that actually in the code we have programmed the QCD corrections for the completely massive case at next-to-leading order, translated to the $\overline{\mathrm{MS}}$ scheme, and interpolated with the massless expression for large Higgs masses, according to the implementation in HDECAY $[60,61]$.
} 
$\Delta_{\mathrm{QED}}^{S}=\Delta_{\mathrm{QED}}^{P}=1+\frac{\alpha}{\pi} Q_{q}^{2}\left(\frac{9}{4}-3 \log \frac{m_{q}^{2}}{M_{H_{i}}^{2}}\right)$,

where $Q_{q}$ denotes the electric charge of the quark $q$. The one-loop SM QCD corrections are similar to the QED corrections, with the replacement of $Q_{q}^{2} \alpha$ by (4/3) $\alpha_{S}\left(M_{H_{i}}^{2}\right)$. It is well-known that the SM QCD corrections are rather large. The large logarithmically enhanced part has been absorbed in Eq. (4.12) into the running $\overline{\mathrm{MS}}$ quark mass $\bar{m}_{q}\left(M_{H_{i}}^{2}\right)$ at the corresponding Higgs mass scale $M_{H_{i}}$ to improve the convergence of the perturbative expansion. The QCD corrections can be taken over from the MSSM case by adapting the Higgs couplings [53-56,62-71]. After subtracting the enhanced part, the remaining QCD correction $\Delta_{\mathrm{QCD}}$ reads

$$
\begin{aligned}
\Delta_{\mathrm{QCD}}^{S}= & \Delta_{\mathrm{QCD}}^{P}=1+\frac{17}{3} \frac{\alpha_{s}\left(M_{H_{i}}^{2}\right)}{\pi}+\left(35.94-1.359 N_{F}\right) \\
& \times\left(\frac{\alpha_{s}\left(M_{H_{i}}^{2}\right)}{\pi}\right)^{2} \\
& +\left(164.14-25.77 N_{F}+0.259 N_{F}^{2}\right)\left(\frac{\alpha_{s}\left(M_{H_{i}}^{2}\right)}{\pi}\right)^{3} \\
& +\left(39.34-220.9 N_{F}+9.685 N_{F}^{2}-0.0205 N_{F}^{3}\right) \\
& \times\left(\frac{\alpha_{s}\left(M_{H_{i}}^{2}\right)}{\pi}\right)^{4},
\end{aligned}
$$

where $N_{F}=5$ active flavors are taken into account. In the CP-conserving case we also include top quark induced corrections $\Delta_{t}^{S / P}$ by adding them to $\Delta_{\mathrm{QCD}}$. They can be taken over from the MSSM case and read [53-56,62-70],

$$
\begin{aligned}
\Delta_{t}^{S}= & \frac{g_{h_{i} t \bar{t}}^{S}}{g_{h_{i} b \bar{b}}^{S}}\left(\frac{\alpha_{S}\left(M_{H_{i}}\right)}{\pi}\right)^{2} \\
& \times\left[1.57-\frac{2}{3} \log \frac{M_{H_{i}}^{2}}{m_{t}^{2}}+\frac{1}{9} \log ^{2} \frac{\bar{m}_{b}^{2}\left(M_{H_{i}}\right)}{M_{H_{i}}^{2}}\right] \\
\Delta_{t}^{P}= & \frac{g_{h_{i} t \bar{t}}^{P}}{g_{h_{i} b \bar{b}}^{P}}\left(\frac{\alpha_{S}\left(M_{H_{i}}\right)}{\pi}\right)^{2} \\
& \times\left[3.83-\log \frac{M_{H_{i}}^{2}}{m_{t}^{2}}+\frac{1}{6} \log ^{2} \frac{\bar{m}_{b}^{2}\left(M_{H_{i}}\right)}{M_{H_{i}}^{2}}\right] .
\end{aligned}
$$

In the decay into a bottom quark pair, the large universal corrections proportional to $\mathcal{O}\left(\alpha_{s} t_{\beta}, \alpha_{b} t_{\beta}\right)$ are resummed into the effective bottom Yukawa couplings $\tilde{g}_{h_{i} b \bar{b}}^{S, P}$, given in Eqs. (4.5) and (4.6), while in the decay into top quarks we use the treelevel values of the effective couplings, i.e.

$\tilde{g}_{h_{i} t \bar{t}}^{S, P}=g_{h_{i} t \bar{t}}^{S, P}$,

with $g_{h_{i} t \bar{t}}$ given in Eq. (4.3). The remaining SUSY-QCD and SUSY-EW corrections are collected in $\delta \mathcal{M}^{\mathrm{rem}, S}, \delta \mathcal{M}^{\mathrm{rem}, P}$ and $\delta \mathcal{M}_{G Z \text {,mix }}$, where

$$
\begin{aligned}
\delta \mathcal{M}^{\mathrm{rem}, S / P}= & \delta \mathcal{M}_{\mathrm{SQCD}}^{\mathrm{rem}, S / P}+\delta \mathcal{M}_{\mathrm{SEW}}^{\mathrm{rem}, S / P} \\
& +\delta \mathcal{M}^{\text {counter }, S / P}
\end{aligned}
$$

with $\delta \mathcal{M}^{\text {counter, } S / P}$ denoting the counterterm contributions. Since in the decay into bottom quarks we have resummed the dominant corrections into the effective couplings, in the remaining SUSY-QCD and SUSY-EW correction we have to subtract these corrections to avoid double counting by adding appropriate counterterms. This is taken care of by the last terms in Eqs. (4.13) and (4.14), respectively, to which we will come back below.

The term $\delta \mathcal{M}_{G Z \text {,mix }}$ is the sum of the contributions from the mixing of the CP-odd component of the Higgs bosons with the neutral Goldstone boson $G$ and with the $Z$ boson, respectively. We use the tree-level masses for the Higgs bosons in the loops in order to ensure the proper cancellation of the UV-divergent pieces, but we use the loopcorrected Higgs boson masses for the external particles in the evaluation of the wave-function renormalization factors, of the amplitudes, and of the decay widths. It is wellknown that the use of loop-corrected Higgs boson masses ${ }^{24}$ for the external particles violates the gauge-parameter independence $[10,11,30,72]$. The Slavnov-Taylor identities ${ }^{25}$ between the renormalized self-energies $\hat{\Sigma}_{h_{i} G}$ and $\hat{\Sigma}_{h_{i} Z}$, with $i=1, \ldots, 5$, are given by

$M_{Z} \hat{\Sigma}_{h_{i} G}\left(p^{2}\right)+i p^{2} \hat{\Sigma}_{h_{i} Z}\left(p^{2}\right)=M_{Z}\left(p^{2}-m_{h_{i}}^{2}\right) f\left(p^{2}\right)$,

where

$$
\begin{aligned}
f\left(p^{2}\right)= & \frac{\alpha}{4 \pi \sin ^{2}\left(2 \theta_{W}\right)} \sum_{j=1}^{6} \\
& \times\left(R_{i 1} R_{j 4}-R_{i 2} R_{j 5}-R_{j 1} R_{i 4}-R_{j 2} R_{i 5}\right) \\
& \times\left(\cos \beta R_{j 1}+\sin \beta R_{j 2}\right) B_{0}\left(p^{2}, M_{Z}^{2}, m_{h_{j}}^{2}\right) .
\end{aligned}
$$

Here, the rotation matrix $R=\mathcal{R} \mathcal{R}^{G}$ with $\mathcal{R}, \mathcal{R}^{G}$ being defined in Eq. (2.8) and $B_{0}$ is the scalar one-loop two-point function. For $p^{2}=m_{h_{i}}^{2}$, the Slavnov-Taylor identities reduce to

$M_{Z} \hat{\Sigma}_{h_{i} G}\left(m_{h_{i}}^{2}\right)=-i m_{h_{i}}^{2} \hat{\Sigma}_{h_{i} Z}\left(m_{h_{i}}^{2}\right)$.

\footnotetext{
24 The resummation through the WFR factor, $\mathbf{Z}^{H}$, causes also violation of the gauge parameter independence as shown in Ref. [74].

25 See Refs. [28,30,72] for the Slavnov-Taylor identities in the MSSM, Ref. [11] for the real NMSSM and Ref. [10] for the relation between unrenomalized self-energies in the complex NMSSM.
} 
Using this relation, the term $\delta \mathcal{M}_{G Z \text {,mix }}$ in general $R_{\xi}$ gauge is given by

$\delta \mathcal{M}_{G Z, \text { mix }}\left(h_{i} \rightarrow q \bar{q}\right)=\frac{I_{2} e m_{q}}{\sin \left(2 \theta_{W}\right)} \frac{\hat{\Sigma}_{h_{i} Z}\left(m_{h_{i}}^{2}\right)}{M_{Z}^{2}}$,

with $I_{2}$ being the isospin of the quark $q$. Using the tree-level masses, the Slavnov-Taylor identities are satisfied. It however causes a mismatch with the phase-space factor (where we use the loop-corrected Higgs boson masses) and with the evaluation of the other amplitudes. We therefore use the loop-corrected masses for the $i$ th external Higgs boson also in these contributions, ${ }^{26}$ i.e.

$\delta \mathcal{M}_{G Z, \text { mix }}\left(h_{i} \rightarrow q \bar{q}\right)=\frac{I_{2} e m_{q}}{\sin \left(2 \theta_{W}\right)} \frac{\hat{\Sigma}_{h_{i} Z}\left(M_{H_{i}}^{2}\right)}{M_{Z}^{2}}$.

The usage of $p^{2} \neq m_{h_{i}}^{2}$ violates the Slavnov-Taylor identity Eq. (4.23), and the possible gauge-parameter dependence due to using $M_{H_{i}}^{2}$ in $\delta \mathcal{M}_{G Z \text {,mix }}$ and in the triangle genuine contributions $\delta \mathcal{M}^{\text {rem, } S / P}$ may be an issue that we investigate in more details in Sect. 5.6. ${ }^{27}$ In order to get a rough estimate of the uncertainty induced by our procedure, we evaluated the loop-corrected decay widths once with $\delta \mathcal{M}_{G Z \text {,mix }}$ evaluated for $m_{h_{i}}^{2}$ and once evaluated for $M_{H_{i}}^{2}$. Denoting by $\Gamma_{\delta \mathcal{M}_{G Z, \text { mix }}\left(M_{H_{i}}^{2}\right)}\left(\Gamma_{\delta \mathcal{M}_{G Z \text {,mix }}\left(m_{h_{i}}^{2}\right)}\right)$ the decay width with $\delta \mathcal{M}_{G Z \text {,mix }}$ evaluated for $M_{H_{i}}^{2}\left(m_{h_{i}}^{2}\right)$ we found relative differences $\left|\Gamma_{\delta \mathcal{M}_{G Z \text {,mix }}\left(M_{H_{i}}^{2}\right)}-\Gamma_{\delta \mathcal{M}_{G Z \text {,mix }}\left(m_{h_{i}}^{2}\right)}\right| / \Gamma_{\delta \mathcal{M}_{G Z, \text { mix }}\left(M_{H_{i}}^{2}\right)}$ of $\mathcal{O}\left(10^{-6}\right)$ for all possible $H_{i} \rightarrow q \bar{q}$ decays for all chosen parameter points used in our scan. The smallness of the relative differences can be understood as follows. The loop corrections to the masses are only significant for the $h_{u}$-like Higgs boson and for the $h_{s}$-like Higgs boson with small mass. These are, however, CP-even-like Higgs bosons. The $\delta \mathcal{M}_{G Z \text {,mix }}$ term on the other hand gives significant contributions only for the doublet CP-odd-like Higgs boson which receives less than one percent loop corrections to its mass. For the singlet CP-odd-like Higgs boson, loop corrections to its mass can be of order $10 \%$ compared to the tree-level mass, however in such cases the mixing between the singlet and doublet CP-odd is small. This leads to the negligible relative differences as observed.

Note that we apply the same method also for the other decays that contain the contribution $\delta \mathcal{M}_{G Z \text {,mix }}$. In Ref. [11],

\footnotetext{
26 This result is identical to the result obtained in the general $R_{\xi}$ gauge provided that the Slavnov-Taylor identities are satisfied with the loopcorrected masses. Another solution has been proposed in [10], where the two-loop term is added such that the Slavnov-Taylor identities are restored with the loop-corrected Higgs masses.

27 We remark that barring this aspect, the full decay amplitude will nonetheless be gauge-dependent due to the usage of loop-corrected external Higgs bosons which induces a mixing of different orders in perturbation theory, see Sect. 5.6.
}

$\delta \mathcal{M}_{G Z \text {,mix }}$ was computed only for the $i$ th tree-level mass eigenstate of the decay $H_{i} \rightarrow q \bar{q}$. This may cause instabilities in the case of large mixing between Higgs boson mass eigenstates at loop-level. We avoid this by multiplying it also with the WFR factor $\mathbf{Z}^{H}$.

The remaining SUSY-QCD and SUSY-EW corrections are computed in the Feynman diagrammatic approach. The corrections consist of the contributions from genuine oneloop diagrams and the counterterms. The counterterms are given by

$\delta \mathcal{M}^{\text {counter }, S / P}\left(h_{i} \rightarrow q \bar{q}\right)=\delta \lambda_{h_{i} q \bar{q}}^{S / P}$,

with the expressions for the scalar and pseudoscalar parts, $\delta \lambda_{h_{i} q \bar{q}}^{S / P}$, reading ${ }^{28}$

$$
\begin{aligned}
\delta \lambda_{h_{i} b \bar{b}}^{S}= & \left(\frac{\delta m_{b}}{m_{b}}-\frac{\delta v}{v}-\frac{\delta c_{\beta}}{c_{\beta}}\right) \frac{\mathcal{R}_{i 1}}{c_{\beta}}+\sum_{k=1}^{5} \frac{\delta Z_{h_{i} h_{k}}}{2} \frac{\mathcal{R}_{k 1}}{c_{\beta}} \\
& +g_{b L}^{h_{i}} \frac{\delta \widetilde{Z}_{b L}}{2}+g_{b L}^{h_{i} *} \frac{\delta \widetilde{Z}_{b R}}{2} \\
\delta \lambda_{h_{i} b \bar{b}}^{P}= & \left(\frac{\delta m_{b}}{m_{b}}-\frac{\delta v}{v}-\frac{\delta c_{\beta}}{c_{\beta}}\right) \mathcal{R}_{i 4} t_{\beta}+\sum_{k=1}^{5} \frac{\delta Z_{h_{i} h_{k}}}{2} \mathcal{R}_{k 4} \\
& +g_{b L}^{h_{i}} \frac{\delta \widetilde{Z}_{b L}}{2}-g_{b L}^{h_{i} *} \frac{\delta \widetilde{Z}_{b R}}{2} \\
\delta \lambda_{h_{i} t \bar{t}}^{S}= & \left(\frac{\delta m_{t}}{m_{t}}-\frac{\delta v}{v}-\frac{\delta s_{\beta}}{s_{\beta}}\right) \frac{\mathcal{R}_{i 2}}{s_{\beta}}+\sum_{k=1}^{5} \frac{\delta Z_{h_{i} h_{k}}}{2} \frac{\mathcal{R}_{k 2}}{s_{\beta}} \\
& +g_{t L}^{h_{i}} \frac{\delta \widetilde{Z}_{t L}}{2}+g_{t L}^{h_{i} *} \frac{\delta \widetilde{Z}_{t R}}{2} \\
\delta \lambda_{h_{i} t \bar{t}}^{P}= & \left(\frac{\delta m_{t}}{m_{t}}-\frac{\delta v}{v}-\frac{\delta s_{\beta}}{s_{\beta}}\right) \frac{\mathcal{R}_{i 4}}{t_{\beta}}+\sum_{k=1}^{5} \frac{\delta Z_{h_{i} h_{k}}}{2} \frac{\mathcal{R}_{k 4}}{t_{\beta}} \\
& +g_{t L}^{h_{i}} \frac{\delta \widetilde{Z}_{t L}}{2}-g_{t L}^{h_{i} *} \frac{\delta \widetilde{Z}_{t R}}{2},
\end{aligned}
$$

with

$g_{b L}^{h_{i}}=\frac{\mathcal{R}_{i 1}}{c_{\beta}}+i \mathcal{R}_{i 4} t_{\beta} \quad$ and $\quad g_{t L}^{h_{i}}=\frac{\mathcal{R}_{i 2}}{s_{\beta}}+i \frac{\mathcal{R}_{i 4}}{t_{\beta}}$.

The counterterm $\delta v$ for the VEV is given by

$$
\frac{\delta v}{v}=-\delta Z_{e}+\frac{c_{\theta_{W}}^{2}}{2 s_{\theta_{W}}^{2}}\left(\frac{\delta M_{W}^{2}}{M_{W}^{2}}-\frac{\delta M_{Z}^{2}}{M_{Z}^{2}}\right)+\frac{\delta M_{W}^{2}}{2 M_{W}^{2}} .
$$

The electric coupling $e$, the $W$ and $Z$ boson masses and $\tan \beta$ are renormalized according to the Higgs sector. The top and bottom quarks are renormalized OS using both the EMT and the Denner descriptions. The terms being related

\footnotetext{
${ }^{28}$ Note, that the angle $\beta$ in the sense of a mixing angle does not get renormalized.
} 
to left-handed and right-handed OS wave function renormalization constants for the quarks $\left(\delta Z_{q L / R}\right)$ and anti-quarks $\left(\delta \bar{Z}_{q L / R}\right)(q=t, b) \operatorname{are}^{29}$

$$
\begin{aligned}
\delta \widetilde{Z}_{q L / R}= & \frac{\delta Z_{q L / R}+\delta \bar{Z}_{q L / R}}{2} \\
= & -\Sigma_{q}^{L / R}\left(m_{q}^{2}\right)-m_{q} \frac{\partial}{\partial p^{2}} \\
& {\left[m_{q}\left(\Sigma_{q}^{L}\left(p^{2}\right)+\Sigma_{q}^{R}\left(p^{2}\right)\right)+\Sigma_{q}^{L s}\left(p^{2}\right)+\Sigma_{q}^{R s}\left(p^{2}\right)\right]_{p^{2}=m_{q}^{2}} . }
\end{aligned}
$$

The $\overline{\mathrm{DR}}$ wave function renormalization constants for the Higgs bosons are denoted by $\delta Z_{h_{i} h_{k}}$. We have checked the UV-finiteness of the SUSY-QCD and SUSY-EW corrections to the decay amplitude.

As mentioned above, in the decay into bottom quarks we have to take care to avoid double counting after resumming the dominant part of the SUSY-QCD and SUSY-EW corrections into the effective bottom coupling. To subtract these contributions we add in the decays into a $b$-quark pair the following counterterms to Eq. (4.13) and Eq. (4.14),

$$
\begin{aligned}
\delta_{\text {sub }}^{S}\left(h_{i} \rightarrow b \bar{b}\right)= & \left(\frac{\mathcal{R}_{i 1}}{c_{\beta}}-\frac{\mathcal{R}_{i 2}}{s_{\beta}}-\frac{\mathcal{R}_{i 3} v}{v_{s}}\right) \operatorname{Re} \Delta_{b} \\
& -\left(\mathcal{R}_{i 4} t_{\beta}+\frac{\mathcal{R}_{i 4}}{t_{\beta}}+\mathcal{R}_{i 5} \frac{v}{v_{s}}\right) \operatorname{Im} \Delta_{b} \\
\delta_{\text {sub }}^{P}\left(h_{i} \rightarrow b \bar{b}\right)= & \left(\mathcal{R}_{i 4} t_{\beta}+\frac{\mathcal{R}_{i 4}}{t_{\beta}}+\mathcal{R}_{i 5} \frac{v}{v_{s}}\right) \operatorname{Re} \Delta_{b} \\
& +\left(\frac{\mathcal{R}_{i 1}}{c_{\beta}}-\frac{\mathcal{R}_{i 2}}{s_{\beta}}-\frac{\mathcal{R}_{i 3} v}{v_{s}}\right) \operatorname{Im} \Delta_{b}
\end{aligned}
$$

where $\Delta_{b}$ equals $\Delta_{b}^{\mathrm{QCD}}-\Delta_{1}$ and $\Delta_{b}^{\text {elw }}$ for the SUSY-QCD and SUSY-EW corrections, respectively. For the decays into a top-quark pair, these contributions are

$\delta_{\mathrm{sub}}^{S / P}\left(h_{i} \rightarrow t \bar{t}\right)=0$.

In the decays into strange quarks we also include the oneloop SUSY-QCD corrections. They are obtained after substituting $\Delta_{b}$ as given in Eq. (4.7) with

$$
\Delta_{s}=\left.\frac{\Delta_{b}^{\mathrm{QCD}}}{1+\Delta_{1}}\right|_{b \rightarrow s} .
$$

The decays into charm quarks are treated as the decays into top quarks, with the appropriate replacements.

The decays into lepton final states $l=e, \mu, \tau$ do not receive QCD corrections at the one-loop order. Their SUSY$\mathrm{EW}$ corrected decay width is given by

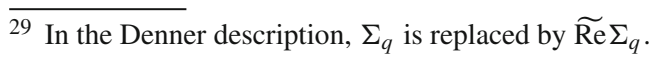

$$
\begin{aligned}
\Gamma\left(H_{i} \rightarrow l \bar{l}\right)= & \frac{G_{F} M_{H_{i}}}{4 \sqrt{2} \pi} m_{l}^{2}\left[\left(1-4 x_{l}\right)^{3 / 2} \Delta_{\mathrm{QED}}^{S} \Gamma_{H_{i} \rightarrow l \bar{l}}^{S}\right. \\
& \left.+\left(1-4 x_{l}\right)^{1 / 2} \Delta_{\mathrm{QED}}^{P} \Gamma_{H_{i} \rightarrow l \bar{l}}^{P}\right]
\end{aligned}
$$

where $x_{l}=m_{l}^{2} / M_{H_{i}}^{2}$. The $\Delta_{\mathrm{QED}}^{S, P}$ are given by Eq. (4.15) after replacing $\left(Q_{q}, m_{q}\right)$ with $\left(Q_{l}, m_{l}\right)$. Furthermore, we resum the dominant SUSY-EW corrections into the effective couplings $\tilde{g}_{h_{i} l l}^{S, P}$. They are obtained from the effective couplings $\tilde{g}_{h_{i} b \bar{b}}$ in Eqs. (4.5) and (4.6) after replacing $\Delta_{b}$ with $\Delta_{l}$, where $\Delta_{l}$ in the complex case is given by

$$
\begin{aligned}
\Delta_{l}= & \frac{e^{2}}{\left(4 \pi s_{W}\right)^{2}} M_{1}^{*} \mu_{\mathrm{eff}}^{*} t_{\beta} I\left(m_{\tilde{l}_{1}}^{2}, m_{\tilde{l}_{2}}^{2},\left|M_{1}\right|^{2}\right) \\
& +\frac{e^{2}}{\left(4 \pi c_{W}\right)^{2}} M_{2}^{*} \mu_{\mathrm{eff}}^{*} t_{\beta} I\left(m_{\tilde{v}_{l}}^{2},\left|M_{2}\right|^{2},\left|\mu_{\mathrm{eff}}\right|^{2}\right) .
\end{aligned}
$$

The contributions $\Gamma_{H_{i} \rightarrow l \bar{l}}^{S, P}$ are obtained from the ones given in Eqs. (4.13) and (4.14) after replacing $q \rightarrow l$.

\subsection{Higgs boson decays into $W^{+} W^{-}$and $Z Z$}

We now address the higher-order corrections to the Higgs boson decays into gauge boson pairs. We consider corrections only for on-shell decays. Off-shell decays are still treated at tree-level as done in NMSSMCALC [14]. The one-loop corrected decay amplitude for the decay of a CP-violating NMSSM Higgs boson $H_{i}(i=1, \ldots, 5)$ into a pair of massive gauge bosons $V=Z, W^{ \pm}, H_{i}(p) \rightarrow V\left(k_{1}\right) V\left(k_{2}\right)$, is given by

$$
\begin{aligned}
& {\left[\sum_{j=1}^{5} \mathbf{Z}_{i j}^{H}\left(\mathcal{M}_{\text {tree }}^{\mu \nu}\left(h_{j} \rightarrow V V\right)+\mathcal{M}_{1 \mathrm{~L}}^{\mu \nu}\left(h_{j} \rightarrow V V\right)\right)\right]} \\
& \quad \epsilon_{\mu}\left(k_{1}\right) \epsilon_{v}\left(k_{2}\right),
\end{aligned}
$$

where $\epsilon_{\mu}\left(k_{1}\right)$ and $\epsilon_{\nu}\left(k_{2}\right)$ are the polarization vectors of the two gauge bosons with four-momenta $k_{1}$ and $k_{2}$, respectively. Note that the contribution arising from the mixing of the $\mathrm{CP}$ odd component of the Higgs bosons with the neutral Goldstone boson $G$ and with the $Z$ boson vanishes. The tree-level amplitudes for the two final-state pairs are

$$
\begin{aligned}
\mathcal{M}_{\text {tree }}^{\mu \nu}\left(h_{j} \rightarrow V V\right) & =g^{\mu \nu} \mathcal{M}_{\text {tree }}\left(h_{j} \rightarrow V V\right), \\
& =g^{\mu \nu} g_{h_{j} V V}\left(c_{\beta} \mathcal{R}_{j 1}+s_{\beta} \mathcal{R}_{j 2}\right)
\end{aligned}
$$

with

$g_{h_{j} V V}=\left\{\begin{array}{ll}\frac{e M_{W}}{s_{\theta_{W}}} & \text { for } V=W \\ \frac{e M_{Z}}{s_{\theta_{W}} c_{\theta_{W}}} & \text { for } V=Z\end{array}\right.$.

And the tensor structure of the NLO corrections is given by

$$
\begin{aligned}
\mathcal{M}_{1 \mathrm{~L}}^{\mu \nu}\left(h_{j} \rightarrow V V\right)= & \left(\mathcal{M}_{1 \mathrm{~L}}^{(1)}+\mathcal{M}_{1 \mathrm{~L}}^{\mathrm{CT}}\right) g^{\mu \nu}+\mathcal{M}_{1 \mathrm{~L}}^{(2)} k_{1}^{\nu} k_{2}^{\mu} \\
& +i \mathcal{M}_{1 \mathrm{~L}}^{(3)} \varepsilon^{\mu \nu \rho \sigma} k_{1 \rho} k_{2 \sigma} .
\end{aligned}
$$


The genuine one-loop triangle diagram contributions are denoted by $\mathcal{M}_{1 \mathrm{~L}}^{(i)}(i=1,2,3)$ and the counterterm contribution by $\mathcal{M}_{1 \mathrm{~L}}^{\mathrm{CT}}$. The term $\mathcal{M}_{1 \mathrm{~L}}^{(3)}$ vanishes in the $\mathrm{CP}$-conserving case.

The decay width for the decay $H_{i} \rightarrow V V$, including the NLO corrections, is given by

$$
\begin{aligned}
& \Gamma\left(H_{i} \rightarrow V V\right) \\
& \quad=R\left(\Gamma^{\text {tree }}\left(H_{i} \rightarrow V V\right)+\Gamma^{1 \mathrm{~L}}\left(H_{i} \rightarrow V V\right)\right),
\end{aligned}
$$

where $R=1 / 2$ for $V=Z$ and $R=1$ for $V=W$. The improved tree-level decay width reads

$$
\begin{aligned}
& \Gamma^{\text {tree }}\left(H_{i} \rightarrow V V\right) \\
& \quad=\frac{\sqrt{r_{V}^{2}-r_{V}} M_{V}^{2}}{4 \pi M_{H_{i}}^{3}} f_{g g}\left|\sum_{j=1}^{5} \mathbf{z}_{i j}^{H} \mathcal{M}_{\text {tree }}\left(h_{j} \rightarrow V V\right)\right|^{2},
\end{aligned}
$$

with

$r_{V}=\frac{M_{H_{i}}^{2}}{4 M_{V}^{2}}$

and

$f_{g g}=4 r_{V}^{2}-4 r_{V}+3$.

The NLO partial width for the Z-boson pair final state contains only virtual contributions,

$\Gamma^{1 \mathrm{~L}}\left(H_{i} \rightarrow Z Z\right)=\Gamma^{\mathrm{virt}}\left(H_{i} \rightarrow Z Z\right)$.

For the $W$-boson pair final state it contains both virtual and real radiation contributions,

$$
\begin{aligned}
& \Gamma^{1 \mathrm{~L}}\left(H_{i} \rightarrow W^{+} W^{-}\right) \\
& \quad=\Gamma^{\mathrm{virt}}\left(H_{i} \rightarrow W^{+} W^{-}\right)+\Gamma^{\mathrm{real}}\left(H_{i} \rightarrow W^{+} W^{-} \gamma\right) .
\end{aligned}
$$

The virtual part is given by

$$
\begin{aligned}
& \Gamma^{\text {virt }}\left(H_{i} \rightarrow V V\right)=\frac{\sqrt{r_{V}^{2}-r_{V}} M_{V}^{2}}{2 \pi M_{H_{i}}^{3}} \\
& \times \operatorname{Re}\left\{\left(\sum_{j=1}^{5} \mathbf{Z}_{i j}^{H} \mathcal{M}_{\text {tree }}\left(h_{j} \rightarrow V V\right)\right)\right. \\
& \times\left[f_{g g} \sum_{l=1}^{5}\left(\mathbf{Z}_{i l}^{H}\right)^{*}\left(\mathcal{M}_{1 \mathrm{~L}}^{(1) *}\left(h_{l} \rightarrow V V\right)+\mathcal{M}_{1 \mathrm{~L}}^{\mathrm{CT} *}\left(h_{l} \rightarrow V V\right)\right)\right. \\
& \left.\left.\quad+M_{H_{i}}^{2} f_{g p} \sum_{l=1}^{5}\left(\mathbf{Z}_{i l}^{H}\right)^{*} \mathcal{M}_{1 \mathrm{~L}}^{(2) *}\left(h_{l} \rightarrow V V\right)\right]\right\},
\end{aligned}
$$

with

$$
f_{g p}=2 r_{V}^{2}-3 r_{V}+1 \text {. }
$$

The formulae for $\mathcal{M}_{1 \mathrm{~L}}^{(1)}$ and $\mathcal{M}_{1 \mathrm{~L}}^{(2)}$ are quite lengthy and we do not display them explicitly here. The counterterm contributions for $V=W$ and $Z$, respectively, read

$$
\begin{aligned}
\mathcal{M}_{1 \mathrm{~L}}^{\mathrm{CT}}\left(h_{j} \rightarrow W^{+} W^{-}\right)= & \mathcal{M}_{\text {tree }}\left(h_{j} \rightarrow W W\right) \\
& \times\left(\delta Z_{e}+\frac{\delta M_{W}^{2}}{2 M_{W}^{2}}-\frac{\delta s_{\theta_{W}}}{s_{\theta_{W}}}+\delta Z_{W}\right) \\
& +\frac{e M_{W}}{s_{\theta_{W}}}\left(-s_{\beta} c_{\beta}^{2} \mathcal{R}_{j 1}+c_{\beta}^{3} \mathcal{R}_{j 2}\right) \delta t_{\beta} \\
& +\frac{1}{2} \sum_{l=1}^{5} \delta Z_{h_{j} h_{l}} \mathcal{M}_{\text {tree }}\left(h_{l} \rightarrow W W\right) \quad(4.53) \\
\mathcal{M}_{\mathrm{lL}}^{\mathrm{CT}}\left(h_{j} \rightarrow Z Z\right)= & \mathcal{M}_{\text {tree }}\left(h_{j} \rightarrow Z Z\right) \\
& \times\left(\delta Z_{e}+\frac{\delta M_{Z}^{2}}{2 M_{Z}^{2}}-\frac{\delta s_{\theta_{W}}}{s_{\theta_{W}}}-\frac{\delta c_{\theta_{W}}}{c_{\theta_{W}}}+\delta Z_{Z}\right) \\
& +\frac{e M_{Z}}{s_{\theta_{W}} c_{\theta_{W}}}\left(-s_{\beta} c_{\beta}^{2} \mathcal{R}_{j 1}+c_{\beta}^{3} \mathcal{R}_{j 2}\right) \delta t_{\beta} \\
& +\frac{1}{2} \sum_{l=1}^{5} \delta Z_{h_{j} h_{l}} \mathcal{M}_{\text {tree }}\left(h_{l} \rightarrow Z Z\right) . \quad(4.54)
\end{aligned}
$$

For the decay $H_{i} \rightarrow W^{+} W^{-}$we have to include the contribution from the radiation of a real photon in order to get an infrared-finite result. This contribution is given by

$$
\begin{aligned}
& \Gamma^{\text {real }}\left(H_{i} \rightarrow W^{+} W^{-} \gamma\right) \\
& \quad=R_{3} \frac{e^{2}}{64 \pi^{3} M_{H_{i}}}\left|\sum_{j=1}^{5} \mathbf{Z}_{i j}^{H} \mathcal{M}_{\text {tree }}\left(h_{j} \rightarrow V V\right)\right|^{2},
\end{aligned}
$$

where

$$
\begin{aligned}
R_{3}= & \frac{1}{M_{W}^{2}}\left[4 M_{W}^{2}\left(-4 r_{W}^{2}+4 r_{W}-3\right)\right. \\
& \times\left(I_{1}+I_{2}+M_{W}^{2} I_{11}+M_{W}^{2} I_{22}\right) \\
& +4 I_{1}^{2}+4 I_{2}^{1}+2 I_{11}^{22}+2 I_{22}^{11}+4 I \\
& \left.+8 M_{W}^{4}\left(8 r_{W}^{3}-12 r_{W}+10 r_{W}-3\right) I_{12}\right] .
\end{aligned}
$$

The formula is in agreement with the result given in Ref. [73]. Here, we have neglected the arguments of the Bremsstrahlung integrals $I_{i_{1}, \ldots, i_{2}}^{j_{1}, \ldots, j_{2}}\left(M_{H_{i}}, M_{W}, M_{W}\right)$ for the sake of readability. In terms of the photon momentum $q$, the $W^{+}$momentum $k_{1}$ and the $W^{-}$momentum $k_{2}$, these integrals are defined as

$$
\begin{aligned}
& I_{i_{1}, \ldots, i_{n}}^{j_{1}, \ldots, j_{m}}\left(M_{H_{i}}, M_{W}, M_{W}\right) \\
& =\frac{1}{\pi^{2}} \int \frac{d^{3} k_{1}}{2 k_{10}} \frac{d^{3} k_{2}}{2 k_{20}} \frac{d^{3} q}{2 q_{0}} \delta\left(k_{0}-k_{1}-k_{2}-q\right) \\
& \quad \times \frac{\left( \pm 2 q k_{j_{1}}\right) \ldots\left( \pm 2 q k_{j_{m}}\right)}{\left( \pm 2 q k_{i_{1}}\right) \ldots\left( \pm 2 q k_{i_{n}}\right)}
\end{aligned}
$$


where $k_{0}$ is the four-momentum of $H_{i}$ and $i_{l}, j_{k}=0,1,2$. The plus signs correspond to $k_{1}, k_{2}$, the minus sign to $k_{0}$. Their analytic expressions are given in Ref. [25].

We have checked the UV finiteness of the NLO decay widths of both the $H_{i} \rightarrow Z Z$ and the $H_{i} \rightarrow W^{+} W^{-}$decays. The IR divergence in the decay $H_{i} \rightarrow W^{+} W^{-}$, however, is more demanding. At strict one-loop level, i.e. one must use the tree-level Higgs boson mass for the external Higgs boson and the unity WFR factor matrix, the IR finiteness is fulfilled. However, the use of the loop-corrected Higgs masses and the WFR factors $\mathbf{Z}_{i j}^{H}$ breaks the IR finiteness, because different orders of perturbation theory are mixed in this case. At tree level there exists a relation between the coupling of the neutral Higgs boson with the charged Goldstone bosons and the coupling of the neutral Higgs boson with the $W$ bosons. Defining the Lagrangian for the interaction between Higgs and Goldstone bosons by

$\mathcal{L}_{h_{j} G^{+} G^{-}}=g_{h_{j} G^{+} G^{-}} h_{j} G^{+} G^{-}+$h.c.,

it is given by

$g_{h_{j} G^{+} G^{-}}=-\frac{m_{h_{j}}^{2}}{g v M_{W}}\left(c_{\beta} \mathcal{R}_{j 1}+s_{\beta} \mathcal{R}_{j 2}\right) g_{h_{j} W^{+} W^{-}}$,

with the tree-level Higgs boson mass $m_{h_{j}}$. In order to obtain an IR-finite result while using the loop-corrected mass $M_{H_{i}}$, we chose to modify the coupling $g_{h_{j} G^{+} G^{-}}$as

$g_{h_{j} G^{+} G^{-}}=-\frac{M_{H_{i}}^{2}}{g v M_{W}}\left(c_{\beta} \mathcal{R}_{j 1}+s_{\beta} \mathcal{R}_{j 2}\right) g_{h_{j} W^{+} W^{-}}$,

where the tree-level mass $m_{h_{j}}^{2}$ has been replaced by the loopcorrected mass $M_{H_{i}}^{2}$ of the decaying Higgs boson $H_{i}$. We verified that the modification of this coupling ensures IR finiteness while not affecting UV finiteness. The same method has been used in Ref. [75]. While taking the loop-corrected mass for the external decaying Higgs boson ensures compatibility with the observation of a $125 \mathrm{GeV}$ SM-like Higgs boson, this approach breaks gauge invariance, however. For more details on this issue, we refer to our investigations in Ref. [74].

For the non-SM-like neutral Higgs bosons, the tree-level coupling $g_{h_{i} V V}$ is in general suppressed, in particular in case the Higgs boson with mass $125.09 \mathrm{GeV}$ behaves very SM-like. In this case, the one-loop corrected decay width $\Gamma^{1 \mathrm{~L}}\left(H_{i} \rightarrow V V\right)$ can be even larger than the tree-level improved one $\Gamma^{\text {tree }}\left(H_{i} \rightarrow V V\right)$. This becomes a problem when the one-loop correction is negative, as then the oneloop corrected partial decay width becomes negative. In this case, we have to include the one-loop squared term, which is formally of higher order. For the decay $H_{i} \rightarrow Z Z$, we will include the one-loop squared contribution. ${ }^{30}$ In particular,

\footnotetext{
30 Note, however, that the thus obtained result has to be taken with caution. The complete two-loop calculation contributes further terms
}

the decay width now is given by

$$
\begin{aligned}
& \Gamma\left(H_{i} \rightarrow Z Z\right)=\frac{1}{2} \\
& \quad\left(\Gamma^{\text {tree }}\left(H_{i} \rightarrow Z Z\right)+\Gamma^{1 \mathrm{~L}}\left(H_{i} \rightarrow Z Z\right)+\Gamma^{1 \mathrm{Ls}}\left(H_{i} \rightarrow Z Z\right)\right),
\end{aligned}
$$

where

$$
\begin{aligned}
\Gamma^{1 \mathrm{Ls}} & \left(H_{i} \rightarrow Z Z\right) \\
= & \frac{\sqrt{r_{V}^{2}-r_{V}} M_{V}^{2}}{4 \pi M_{H_{i}}^{3}}\left\{\left(\sum_{j=1}^{5} \mathbf{Z}_{i j}^{H} \mathcal{M}_{1 \mathrm{~L}}^{(1)+\mathrm{CT}}\left(h_{j} \rightarrow Z Z\right)\right)\right. \\
& \times\left(f_{g g} \sum_{l=1}^{5} \mathbf{Z}_{i l}^{H} \mathcal{M}_{1 \mathrm{~L}}^{(1)+\mathrm{CT}}\left(h_{l} \rightarrow Z Z\right)\right. \\
& \left.+M_{H_{i}}^{2} f_{g p} \sum_{l=1}^{5} \mathbf{Z}_{i l}^{H} \mathcal{M}_{1 \mathrm{~L}}^{(2)}\left(h_{l} \rightarrow Z Z\right)\right)^{*} \\
& +M_{H_{i}}^{4}\left(r_{V}-1\right)^{2}\left(\sum_{j=1}^{5} \mathbf{Z}_{i j}^{H} \mathcal{M}_{1 \mathrm{~L}}^{(2)}\left(h_{j} \rightarrow Z Z\right)\right) \\
& \times\left(\sum_{l=1}^{5} \mathbf{Z}_{i l}^{H} \mathcal{M}_{1 \mathrm{~L}}^{(2)}\left(h_{l} \rightarrow Z Z\right)\right)^{*} \\
& +M_{H_{i}}^{4} \frac{r_{V}-1}{r_{V}}\left(\sum_{j=1}^{5} \mathbf{Z}_{i j}^{H} \mathcal{M}_{1 \mathrm{~L}}^{(3)}\left(h_{j} \rightarrow Z Z\right)\right) \\
& \left.\times\left(\sum_{l=1}^{5} \mathbf{Z}_{i l}^{H} \mathcal{M}_{1 \mathrm{~L}}^{(3)}\left(h_{l} \rightarrow Z Z\right)\right)^{*}\right\}
\end{aligned}
$$

where $\mathcal{M}_{1 \mathrm{~L}}^{(1)+\mathrm{CT}}$ is the sum of $\mathcal{M}_{1 \mathrm{~L}}^{(1)}+\mathcal{M}_{1 \mathrm{~L}}^{\mathrm{CT}}$. For the decay $H_{i} \rightarrow W^{+} W^{-}$, the form factor $\mathcal{M}_{1 \mathrm{~L}}^{(1)+\mathrm{CT}}$ contains IR divergences so that we cannot treat it in the same way as in the decay $H_{i} \rightarrow Z Z$. Note, however, that the $1 \mathrm{~L}$ decay width $\Gamma^{1 \mathrm{~L}}\left(H_{i} \rightarrow W W\right)$ can always be divided into three parts that are separately UV and IR finite: the (s)fermion contribution arising from loops containing SM model fermions and their superpartners, the chargino/neutralino contribution from loops with internal charginos and neutralinos and the gauge/Higgs contribution from loops with gauge and Higgs

that might cause the complete two-loop result to differ considerably from the result obtained in the here applied approximation. Moreover, the inclusion of (part of) the two-loop corrections explicitly includes a dependence on the renormalization scheme chosen at one-loop order that would need to be cancelled by transforming the input parameters appropriately so as not to become inconsistent. We still use this approach in order to obtain physical, i.e. positive, partial decay widths and hence physical branching ratios. Since the partial decay width is suppressed here anyway, the effect of the difference between the approximation and the full two-loop result on the branching ratio is expected to be subleading. Still, the code NMSSMCALC will print out a warning to make the user aware of this issue. 
particles. In many cases the dominant contribution is the (s)fermion part. That was also observed in the MSSM case $[75,76]$. We therefore add to the one-loop corrected decay width $H_{i} \rightarrow W^{+} W^{-}$the one-loop squared contribution from the (s)fermion part only. This part is IR finite as it solely involves fermions and sfermions but no photons. Both for the decays into $Z Z$ and into $W W$ we include the respective one-loop squared terms in case the one-loop contribution is larger than $80 \%$ of the tree-level decay width.

\subsection{Higgs boson decays into a $Z$ boson and a Higgs boson}

The one-loop corrected amplitude for the decay of a heavy Higgs boson $H_{i}$ with four-momentum $p$ into a light Higgs boson $H_{j}$ and a $Z$ boson, with four-momenta $k_{1}$ and $k_{2}$, respectively, $H_{i}(p) \rightarrow H_{j}\left(k_{1}\right) Z\left(k_{2}\right)$, can be written as

$$
M_{1 \mathrm{~L}}\left(H_{i} \rightarrow H_{j} Z\right)=\epsilon_{\mu}\left(k_{2}\right) p^{\mu} \mathcal{M}_{1 \mathrm{~L}}\left(H_{i} \rightarrow H_{j} Z\right),
$$

where

$$
\begin{aligned}
& \mathcal{M}_{1 \mathrm{~L}}\left(H_{i} \rightarrow H_{j} Z\right)=\sum_{k^{\prime}, l^{\prime}=1}^{5} \mathbf{Z}_{i k^{\prime}}^{H} \mathbf{Z}_{j l^{\prime}}^{H} \\
& \quad \times\left(\mathcal{M}_{h_{k^{\prime}} h_{l^{\prime}} Z}^{(0)}+\mathcal{M}_{h_{k^{\prime}} h_{l^{\prime}} Z}^{(1)}+\mathcal{M}_{h_{k^{\prime}} h_{l^{\prime}} Z}^{G Z, \text { mix }}\right) .
\end{aligned}
$$

The tree-level expression $\mathcal{M}_{h_{i} h_{j} Z}^{(0)}$ reads

$$
\begin{aligned}
\mathcal{M}_{h_{i} h_{j} Z}^{(0)}= & \frac{e}{s_{\theta_{W}} c_{\theta_{W}}}\left(s_{\beta}\left(\mathcal{R}_{i 4} \mathcal{R}_{j 1}-\mathcal{R}_{i 1} \mathcal{R}_{j 4}\right)\right. \\
& \left.+c_{\beta}\left(-\mathcal{R}_{i 4} \mathcal{R}_{j 2}+\mathcal{R}_{i 2} \mathcal{R}_{j 4}\right)\right),
\end{aligned}
$$

and the one-loop term $\mathcal{M}_{h_{i} h_{j} Z}^{(1)}$ consists of the genuine oneloop diagram contribution and the counterterm part given by

$$
\begin{aligned}
\mathcal{M}_{h_{i} h_{j} Z}^{\mathrm{CT}}= & \frac{1}{2} \sum_{i^{\prime}=1}^{5}\left(\delta Z_{h_{i} h_{i^{\prime}}} \mathcal{M}_{h_{i^{\prime}} h_{j} Z}^{(0)}+\delta Z_{h_{j} h_{i^{\prime}}} \mathcal{M}_{h_{i} h_{i^{\prime}} Z}^{(0)}\right) \\
& +\frac{1}{2} \mathcal{M}_{h_{i} h_{j} Z}^{(0)} \delta Z_{Z} \\
& +\mathcal{M}_{h_{i} h_{j} Z}^{(0)}\left(\delta Z_{e}-\frac{c_{2 \theta_{W}} \delta s_{\theta_{W}}}{s_{\theta_{W}} c_{\theta_{W}}^{2}}\right)
\end{aligned}
$$

Also here, the contribution from the one-loop diagrams with the transition $h_{i} \rightarrow Z(G), \mathcal{M}_{h_{i} h_{j} Z}^{G Z \text {,mix }}$, is calculated using the same technique as discussed in Sect. 4.1. The improved tree-level decay width is given by

$$
\Gamma^{\text {tree }}\left(H_{i} \rightarrow H_{j} Z\right)=R_{H H Z}\left|\sum_{i^{\prime}, j^{\prime}=1}^{5} \mathbf{Z}_{i i^{\prime}}^{H} \mathbf{z}_{j j^{\prime}}^{H} \mathcal{M}_{h_{i^{\prime}} h_{j^{\prime}} Z}^{(0)}\right|^{2}
$$

and the NLO decay width by

$$
\Gamma^{\mathrm{NLO}}\left(H_{i} \rightarrow H_{j} Z\right)=R_{H H Z}\left|\mathcal{M}_{1 \mathrm{~L}}\left(H_{i} \rightarrow H_{j} Z\right)\right|^{2},
$$

with the 2-particle phase-space factor

$$
\begin{aligned}
R_{H H Z} & =\frac{\lambda^{3 / 2}\left(M_{H_{i}}^{2}, M_{H_{j}}^{2}, M_{Z}^{2}\right)}{64 \pi M_{H_{i}}^{3} M_{Z}^{2}}, \\
\lambda(x, y, z) & =x^{2}+y^{2}+z^{2}-2 x y-2 x z-2 y z .
\end{aligned}
$$

Since the formulae for the 1-loop amplitudes are quite lengthy we do not display them explicitly here. Note that, as in the decay into massive gauge bosons, in Eq. (4.68) we also included, keeping in mind the caveat mentioned there, one-loop contributions squared as the one-loop corrections can be large and negative.

\subsection{Higgs boson decays into neutralinos and charginos}

The couplings of a neutral Higgs boson $h_{i}$ with the electroweakinos can be defined as

$$
-i \frac{e}{2 s_{\theta_{W}}}\left(g_{h_{i} \tilde{\chi}_{j} \tilde{\chi}_{k}}^{L} P_{L}+g_{h_{i} \tilde{\chi}_{k} \tilde{\chi}_{j}}^{R} P_{R}\right)
$$

where $\tilde{\chi}$ stands generically for the neutralinos and charginos and $g_{h_{i} \tilde{\chi}_{k} \tilde{\chi}_{j}}^{R}=\left(g_{h_{i} \tilde{\chi}_{k} \tilde{\chi}_{j}}^{L}\right)^{*}$. At tree level, the left- and righthanded coefficients for the Higgs-chargino couplings are given in terms of [14]

$$
\begin{aligned}
g_{h_{i} \tilde{\chi}_{j}^{+} \tilde{\chi}_{k}^{-}}^{L}= & \frac{\lambda v\left(\mathcal{R}_{i 3}+i \mathcal{R}_{i 5}\right) U_{j 2}^{*} V_{k 2}^{*} e^{i \varphi_{s}}}{\sqrt{2} M_{Z} c_{\theta_{W}}} \\
& +\sqrt{2} U_{j 1}^{*} V_{k 2}^{*} e^{-i \varphi_{u}}\left(\mathcal{R}_{i 2}-i c_{\beta} \mathcal{R}_{i 4}\right) \\
& +\sqrt{2} U_{j 2}^{*} V_{k 1}^{*}\left(\mathcal{R}_{i 1}-i s_{\beta} \mathcal{R}_{i 4}\right)
\end{aligned}
$$

where $i=1, \ldots, 5, j, k=1,2$, and for the Higgs-neutralino couplings we have

$$
\begin{aligned}
g_{h_{i} \tilde{\chi}_{l}^{0} \tilde{\chi}_{m}^{0}}^{L}= & {\left[\frac{1}{c_{\theta_{W}}} N_{l 3}^{*}\left(c_{\theta_{W}} N_{m 2}^{*}-s_{\theta_{W}} N_{m 1}^{*}\right)\left(\mathcal{R}_{i 1}-i s_{\beta} \mathcal{R}_{i 4}\right)\right.} \\
& -\frac{1}{c_{\theta_{W}}} N_{l 4}^{*}\left(c_{\theta_{W}} N_{m 2}^{*}-s_{\theta_{W}} N_{m 1}^{*}\right) e^{-i \varphi_{u}}\left(\mathcal{R}_{i 2}-i c_{\beta} \mathcal{R}_{i 4}\right) \\
& -\frac{\lambda v N_{l 5}^{*} N_{m 3}^{*} e^{i \varphi_{u}}\left(\mathcal{R}_{i 2}+i c_{\beta} \mathcal{R}_{i 4}\right)}{\sqrt{2} M_{Z} c_{\theta_{W}}} \\
& -\frac{\lambda v N_{l 5}^{*} N_{m 4}^{*}\left(\mathcal{R}_{i 1}+i s_{\beta} \mathcal{R}_{i 4}\right)}{\sqrt{2} M_{Z} c_{\theta_{W}}} \\
& +\frac{v\left(\mathcal{R}_{i 3}+i \mathcal{R}_{i 5}\right) e^{i \varphi_{s}}\left(2 \kappa N_{l 5}^{*} N_{m 5}^{*}-\lambda N_{l 4}^{*} N_{m 3}^{*}\right)}{\sqrt{2} M_{Z} c_{\theta_{W}}} \\
& +l \leftrightarrow m],
\end{aligned}
$$


with $l, m=1, \ldots, 5$. The decay width for the decay of a Higgs boson $H_{i}$ into a neutralino pair or a chargino pair including higher order corrections is given by

$$
\begin{aligned}
& \Gamma\left(H_{i} \rightarrow \tilde{\chi}_{j} \tilde{\chi}_{k}\right) \\
& \quad=R\left(\Gamma^{\text {tree }}\left(H_{i} \rightarrow \tilde{\chi}_{j} \tilde{\chi}_{k}\right)+\Gamma^{1 \mathrm{~L}}\left(H_{i} \rightarrow \tilde{\chi}_{j} \tilde{\chi}_{k}\right)\right),
\end{aligned}
$$

where $R=1 / 2$ for identical final states and $R=1$ otherwise. The improved tree-level decay width reads

$$
\begin{aligned}
\Gamma^{\text {tree }} & \left(H_{i} \rightarrow \tilde{\chi}_{j} \tilde{\chi}_{k}\right) \\
= & R_{2}\left(M_{H_{i}}^{2}, M_{\tilde{\chi}_{j}}^{2}, M_{\tilde{\chi}_{k}}^{2}\right) \\
& \times\left[\left(M_{H_{i}}^{2}-M_{\tilde{\chi}_{j}}^{2}-M_{\tilde{\chi}_{k}}^{2}\right)\left(\left|\mathcal{M}_{H_{i} \tilde{\chi}_{j} \tilde{\chi}_{k}}^{L, 0}\right|^{2}+\left|\mathcal{M}_{H_{i} \tilde{\chi}_{j} \tilde{\chi}_{k}}^{R}\right|^{2}\right)\right. \\
& \left.-4 M_{\tilde{\chi}_{j}} M_{\tilde{\chi}_{k}} \operatorname{Re}\left[\mathcal{M}_{H_{i} \tilde{\chi}_{j} \tilde{\chi}_{k}}^{L, 0} \mathcal{M}_{H_{i} \tilde{\chi}_{j} \tilde{\chi}_{k}}^{R, 0 *}\right]\right],
\end{aligned}
$$

where the 2-body phase space factor is

$R_{2}(x, y, z)=\frac{\lambda^{1 / 2}(x, y, z)}{16 \pi x^{3 / 2}}$,

in terms of the improved tree-level amplitude

$\mathcal{M}_{H_{i} \tilde{\chi}_{j} \tilde{\chi}_{k}}^{L / R, 0}=\sum_{i^{\prime}=1}^{5} \mathbf{Z}_{i i,}^{H} \frac{e}{2 s_{\theta_{W}}} g_{h_{i^{\prime}} \tilde{\chi}_{j} \tilde{\chi}_{k}}^{L / R}$.

The one-loop decay width for the decay into a neutralino pair is given by

$$
\Gamma^{1 \mathrm{~L}}\left(H_{i} \rightarrow \tilde{\chi}_{j}^{0} \tilde{\chi}_{k}^{0}\right)=\Gamma^{\mathrm{virt}}\left(H_{i} \rightarrow \tilde{\chi}_{j}^{0} \tilde{\chi}_{k}^{0}\right)
$$

and for the decay into a chargino pair it is

$$
\begin{aligned}
& \Gamma^{1 \mathrm{~L}}\left(H_{i} \rightarrow \tilde{\chi}_{j}^{+} \tilde{\chi}_{k}^{-}\right) \\
& \quad=\Gamma^{\mathrm{virt}}\left(H_{i} \rightarrow \tilde{\chi}_{j}^{+} \tilde{\chi}_{k}^{-}\right)+\Gamma^{\mathrm{real}}\left(H_{i} \rightarrow \tilde{\chi}_{j}^{+} \tilde{\chi}_{k}^{-}\right),
\end{aligned}
$$

where the virtual contribution can be cast into the form

$$
\begin{aligned}
& \Gamma^{\mathrm{virt}}\left(H_{i} \rightarrow \tilde{\chi}_{j} \tilde{\chi}_{k}\right) \\
& =2 R_{2}\left(M_{H_{i}}^{2}, M_{\tilde{\chi}_{j}}^{2}, M_{\tilde{\chi}_{k}}^{2}\right) \\
& \times\left[\left(M_{H_{i}}^{2}-M_{\tilde{\chi}_{j}}^{2}-M_{\tilde{\chi}_{k}}^{2}\right)\right. \\
& \times \operatorname{Re}\left(\mathcal{M}_{H_{i} \tilde{\chi}_{j} \tilde{\chi}_{k}}^{L, 0} \mathcal{M}_{H_{i} \tilde{\chi}_{j} \tilde{\chi}_{k}}^{L, 1 *}+\mathcal{M}_{H_{i} \tilde{\chi}_{j} \tilde{\chi}_{k}}^{R, 0} \mathcal{M}_{H_{i} \tilde{\chi}_{j} \tilde{\chi}_{k}}^{R, 1 *}\right)-2 M_{\tilde{\chi}_{j}} M_{\tilde{\chi}_{k}} \\
& \left.\times \operatorname{Re}\left[\mathcal{M}_{H_{i} \tilde{\chi}_{j} \tilde{\chi}_{k}}^{L, 0} \mathcal{M}_{H_{i} \tilde{\chi}_{j} \tilde{\chi}_{k}}^{R, 1 *}+\mathcal{M}_{H_{i} \tilde{\chi}_{j} \tilde{\tilde{k}}_{k}}^{R, 0} \mathcal{M}_{H_{i} \tilde{\chi}_{j} \tilde{x}_{k}}^{L, 1 *}\right]\right],
\end{aligned}
$$

with the left- and right-handed one-loop amplitudes containing genuine triangle, counterterm and ' $G Z$, mix' contributions,

$$
\begin{aligned}
\mathcal{M}_{H_{i} \tilde{\chi}_{j} \tilde{\chi}_{k}}^{L / R}= & \sum_{i^{\prime}=1}^{5} \mathbf{Z}_{i i^{\prime}}^{H} \\
& \times\left(\mathcal{M}_{h_{i^{\prime}} \tilde{\chi}_{j} \tilde{\chi}_{k}}^{L / R, \Delta}+\mathcal{M}_{h_{i^{\prime}} \tilde{\chi}_{j} \tilde{\chi}_{k}}^{L / R, \mathrm{CT}}+\mathcal{M}_{h_{i^{\prime}} \tilde{\chi}_{j} \tilde{\chi}_{k}}^{L / R, G Z, \operatorname{mix}}\right) .
\end{aligned}
$$

We do not display explicitly here the lengthy expressions for the triangle and ' $G Z$, mix' contributions. Note that the ' $G Z$, mix' contributions are computed using the same technique as discussed in Sect. 4.1. The explicit expressions of the counterterm amplitudes for the decays into neutralinos are

$$
\begin{aligned}
& \mathcal{M}_{h_{i} \tilde{\chi}_{j}^{0} \tilde{\chi}_{k}^{0}}^{L, \mathrm{CT}}=\frac{e}{4 s_{\theta_{W}}}\left[\sum_{i^{\prime}=1,5} g_{h_{i^{\prime}} \tilde{\chi}_{j}^{0} \tilde{\chi}_{k}^{0}}^{L} \delta Z_{h_{i} h_{i^{\prime}}}\right. \\
& +\sum_{j^{\prime}=1,5} g_{h_{i} \tilde{\chi}_{j^{\prime}}^{0} \tilde{\chi}_{k}^{\delta}}^{L} \delta Z_{L, j^{\prime} j}^{\tilde{\chi}^{0}} \\
& \left.+\sum_{k^{\prime}=1,5} g_{h_{i} \tilde{\chi}_{j}^{0} \tilde{x}_{k^{\prime}}^{0}}^{L} \delta \bar{Z}_{L, k k^{\prime}}^{\tilde{\chi}^{0}}\right] \\
& +\frac{e}{2 s_{\theta_{W}}} g_{h_{i} \tilde{\chi}_{j}^{0} \tilde{\chi}_{k}^{0}}\left(\delta Z_{e}-\frac{\delta s_{\theta_{W}}}{s_{\theta_{W}}}\right)+\frac{e}{2 s_{\theta_{W}}} \\
& \times\left[-N_{l 3}^{*} N_{m 1}^{*} \delta t_{\theta_{W}}\left(\mathcal{R}_{i 1}-i s_{\beta} \mathcal{R}_{i 4}\right)\right. \\
& +N_{l 4}^{*} N_{m 1}^{*} e^{-i \varphi_{u}} \delta t_{\theta_{W}}\left(\mathcal{R}_{i 2}-i c_{\beta} \mathcal{R}_{i 4}\right) \\
& -\frac{\lambda v}{\sqrt{2} M_{Z} c_{\theta_{W}}}\left(N_{l 5}^{*} N_{m 3}^{*} e^{i \varphi_{u}}\right. \\
& \times\left(\mathcal{R}_{i 2}+i c_{\beta} \mathcal{R}_{i 4}\right)+N_{l 5}^{*} N_{m 4}^{*}\left(\mathcal{R}_{i 1}+i s_{\beta} \mathcal{R}_{i 4}\right) \\
& \left.+\left(\mathcal{R}_{i 3}+i \mathcal{R}_{i 5}\right) e^{i \varphi_{s}} N_{l 4}^{*} N_{m 3}^{*}\right) \\
& \times\left(\frac{\delta v}{v}+\frac{\delta \lambda}{\lambda}-\frac{\delta M_{Z}^{2}}{2 M_{Z}^{2}}-\frac{\delta c_{\theta_{W}}}{c_{\theta_{W}}}\right) \\
& +\frac{\sqrt{2} v \kappa\left(\mathcal{R}_{i 3}+i \mathcal{R}_{i 5}\right) e^{i \varphi_{s}} N_{l 5}^{*} N_{m 5}^{*}}{M_{Z} c_{\theta_{W}}} \\
& \left.\times\left(\frac{\delta v}{v}+\frac{\delta \kappa}{\kappa}-\frac{\delta M_{Z}^{2}}{2 M_{Z}^{2}}-\frac{\delta c_{\theta_{W}}}{c_{\theta_{W}}}\right)+l \leftrightarrow m\right],
\end{aligned}
$$

and for the decays into charginos they read

$$
\begin{aligned}
& \mathcal{M}_{h_{i} \tilde{x}_{j}^{+} \tilde{x}_{k}^{-}}^{L, \mathrm{CT}}=\frac{e}{4 s_{\theta_{W}}} \\
& \times\left[\sum_{i^{\prime}=1,5} g_{h_{i^{\prime}} \tilde{x}_{j}^{+} \tilde{\chi}_{k}^{-}}^{L} \delta Z_{h_{i} h_{i^{\prime}}}+\sum_{j^{\prime}=1,2} g_{h_{i} \tilde{x}_{j^{\prime}}^{+} \tilde{z}_{k}^{L}}^{L} \delta Z_{L, j^{\prime} j}^{\tilde{x}^{+}}\right. \\
& \left.\quad+\sum_{k^{\prime}=1,2} g_{h_{i} \tilde{x}_{j}^{+} \tilde{\tilde{k}}_{k^{\prime}}^{-}}^{L} \delta \bar{Z}_{L, k k^{\prime}}^{\tilde{x}^{+}}\right]
\end{aligned}
$$




$$
\begin{aligned}
& +\frac{e}{\sqrt{2} s_{\theta_{W}}}\left(U_{j 1}^{*} V_{k 2}^{*} e^{-i \varphi_{u}}\left(\mathcal{R}_{i 2}-i c_{\beta} \mathcal{R}_{i 4}\right)+U_{j 2}^{*} V_{k 1}^{*}\left(\mathcal{R}_{i 1}-i s_{\beta} \mathcal{R}_{i 4}\right)\right) \\
& \times\left(\delta Z_{e}-\frac{\delta s_{\theta_{W}}}{s_{\theta_{W}}}\right)+\frac{U_{j 2}^{*} V_{k 2}^{*} e^{i \varphi_{s}}\left(\mathcal{R}_{i 3}+i \mathcal{R}_{i 5}\right)}{\sqrt{2}} \delta \lambda
\end{aligned}
$$

The right-handed counterterm amplitudes are equal to the complex conjugate of the corresponding left-handed parts after interchanging the indices of the charginos and neutralinos in the final state. The real photon contribution for the decays into a chargino pair is expressed in terms of the Bremsstrahlung integrals as

$$
\begin{aligned}
& \Gamma^{\text {real }}\left(H_{i} \rightarrow \tilde{\chi}_{j}^{+} \tilde{\chi}_{k}^{-}\right)=\frac{e^{2}}{32 \pi^{3} M_{H_{i}}} \\
& \times\left\{2\left(\left|\mathcal{M}_{H_{i} \tilde{\chi}_{j} \tilde{\chi}_{k}}^{L, 0}\right|^{2}+\left|\mathcal{M}_{H_{i} \tilde{\chi}_{j} \tilde{\chi}_{k}}^{R, 0}\right|^{2}\right)\left(I_{2}^{1}+I_{1}^{2}+2 I\right)\right. \\
& -\left(I_{1}+I_{2}-2\left(M_{H_{i}}^{2}-M_{\tilde{\chi}_{j}^{+}}^{2}-M_{\tilde{\chi}_{k}^{-}}^{2}\right) I_{12}+2 M_{\tilde{\chi}_{j}^{+}}^{2} I_{11}+2 M_{\tilde{\chi}_{k}^{-}}^{2} I_{22}\right) \\
& \times\left[\left(M_{H_{i}}^{2}-M_{\tilde{\chi}_{j}^{+}}^{2}-M_{\tilde{\chi}_{k}^{-}}^{2}\right)\left(\left|\mathcal{M}_{H_{i} \tilde{\chi}_{j} \tilde{\chi}_{k}}^{L, 0}\right|^{2}+\left|\mathcal{M}_{H_{i} \tilde{\chi}_{j} \tilde{x}_{k}}^{R, 0}\right|^{2}\right)\right. \\
& \left.\left.-4 M_{\tilde{\chi}_{j}^{+}} M_{\tilde{\chi}_{k}^{-}} \operatorname{Re}\left[\mathcal{M}_{H_{i} \tilde{\chi}_{j} \tilde{\chi}_{k}}^{L, 0} \mathcal{M}_{H_{i} \tilde{\chi}_{j} \tilde{\chi}_{k}}^{R, 0 *}\right]\right]\right\},
\end{aligned}
$$

where the arguments of the Bremsstrahlung integrals $I_{i_{1}, \ldots, i_{n}}^{j_{1}, \ldots, j_{m}}$ $\left(M_{H_{i}}, M_{\tilde{\chi}_{j}^{+}}, M_{\tilde{\chi}_{k}^{-}}\right)$have been neglected. Note that we use the loop-corrected masses for the external Higgs boson and the external charginos and neutralinos in the tree-level, virtual and real contributions. However, for particles inside loops we use the tree-level masses and tree-level couplings. This does not affect the UV-finiteness but can break the IR-finiteness in the decay into a pair of charginos. We overcome this problem by replacing the tree-level mass of the chargino in the loop diagrams with a photon by the corresponding loop-corrected chargino mass. Our treatment is different from Ref. [9] where the authors define an IR divergent counterterm to cancel the mismatch between the real and virtual contributions. Note finally, that in case the NLO decay width into neutralino final states becomes negative, the improved tree-level decay width is calculated instead in NMSSMCALCEW, including the $\mathbf{Z}^{H}$ factor.

\subsection{Higgs boson decays into squark pairs}

The NLO corrections to the decay of a neutral Higgs boson into a squark-antisquark pair consist of the QCD and EW corrections. In the CP-conserving NMSSM, the NLO corrections to the decay of a $\mathrm{CP}$-odd Higgs boson into a stop pair have been calculated and discussed in Ref. [6]. We extend this computation to the $\mathrm{CP}$-violating case and include also the decay into a sbottom-antisbottom pair in this paper. The NLO QCD corrections are positive and large. They can be larger than $100 \%$ as observed in Ref. [6] while the EW correction are negative and can be of up to $-40 \%$. In our calculation, we have implemented both the OS and the $\overline{\mathrm{DR}}$ scheme. We have three options here. First, the seven parameters are renormalized in the OS scheme. Second, the parameters of the stop sector, $m_{t}, m_{\tilde{Q}_{3}}, m_{\tilde{t}_{R}}, A_{t}$, are renormalized in the OS scheme while the remaining parameters, $m_{b}, m_{\tilde{b}_{R}}, A_{b}$, are renormalized in the $\overline{\mathrm{DR}}$ scheme. ${ }^{31}$ Third, all parameters are renormalized in the $\overline{\mathrm{DR}}$ scheme. The loop-corrected decay width is decomposed into the improved tree-level, one-loop QCD and one-loop EW decay widths,

$$
\begin{gathered}
\Gamma\left(H_{i} \rightarrow \tilde{q}_{j} \tilde{q}_{k}^{*}\right)=\Gamma^{\mathrm{tree}}\left(H_{i} \rightarrow \tilde{q}_{j} \tilde{q}_{k}^{*}\right)+\Gamma_{\mathrm{QCD}}^{(1)}\left(H_{i} \rightarrow \tilde{q}_{j} \tilde{q}_{k}^{*}\right) \\
+\Gamma_{\mathrm{EW}}^{(1)}\left(H_{i} \rightarrow \tilde{q}_{j} \tilde{q}_{k}^{*}\right) .
\end{gathered}
$$

Denoting the color factor by $N_{F}$, with $N_{F}=3$, the improved tree-level decay width is given by

$$
\begin{aligned}
& \Gamma^{\text {tree }}\left(H_{i} \rightarrow \tilde{q}_{j} \tilde{q}_{k}^{*}\right) \\
& \quad=N_{F} R_{2}\left(M_{H_{i}}^{2}, M_{\tilde{q}_{j}}^{2}, M_{\tilde{q}_{k}}^{2}\right)\left|\mathcal{M}_{H_{i} \tilde{q}_{j} \tilde{q}_{k}^{*}}^{0}\right|^{2},
\end{aligned}
$$

in terms of the improved tree-level amplitude

$\mathcal{M}_{H_{i} \tilde{q}_{j} \tilde{q}_{k}^{*}}^{0}=\sum_{i_{1}=1}^{5} \mathbf{Z}_{i i^{\prime}}^{H} g_{h_{i^{\prime}} \tilde{q}_{j} \tilde{q}_{k}^{*}}$.

The tree-level Higgs-squark-squark couplings are given by [14]

$$
\begin{aligned}
g_{h_{i} \tilde{t}_{j} \tilde{t}_{k}^{*}}= & \frac{e M_{Z}}{s_{\theta_{W}} c_{\theta_{W}}}\left[\frac{m_{t}^{2} \mathcal{R}_{i 2}\left(U_{j 1}^{\tilde{t} *} U_{k 1}^{\tilde{t}}+U_{j 2}^{\tilde{t} *} U_{k 2}^{\tilde{t}}\right)}{s_{\beta} M_{Z}^{2}}\right. \\
& +\frac{m_{t}\left(U_{j 2}^{\tilde{t} *} U_{k 1}^{\tilde{t}} F_{1}+U_{j 1}^{\tilde{t} *} U_{k 2}^{\tilde{t}} F_{1}^{*}\right)}{2 s_{\beta} M_{Z}^{2}} \\
& +\frac{1}{6}\left(c_{\beta} \mathcal{R}_{i 1}-s_{\beta} \mathcal{R}_{i 2}\right) \\
& \left.\times\left(\left(4 c_{\theta_{W}}^{2}-1\right) U_{j 1}^{\tilde{t} *} U_{k 1}^{\tilde{t}}+4 s_{\theta_{W}}^{2} U_{j 2}^{\tilde{t} *} U_{k 2}^{\tilde{t}}\right)\right]
\end{aligned}
$$

$$
\begin{aligned}
g_{h_{i} \tilde{b}_{j} \tilde{b}_{k}^{*}}= & \frac{e M_{Z}}{s_{\theta_{W}} c_{\theta_{W}}}\left[\frac{m_{b}^{2} \mathcal{R}_{i 1}\left(U_{j 1}^{\tilde{b} *} U_{k 1}^{\tilde{b}}+U_{j 2}^{\tilde{b} *} U_{k 2}^{\tilde{b}}\right)}{c_{\beta} M_{Z}^{2}}\right. \\
& +\frac{m_{b}\left(U_{j 2}^{\tilde{b} *} U_{k 1}^{\tilde{b}} F_{2}+U_{j 1}^{\tilde{b} *} U_{k 2}^{\tilde{b}} F_{2}^{*}\right)}{2 c_{\beta} M_{Z}^{2}}
\end{aligned}
$$

\footnotetext{
${ }^{31}$ While it is possible to choose different renormalization schemes of the (s)top sector in the loop-corrected Higgs mass and decay width calculations, we recommend users to select the same renormalization scheme in order to avoid spurious contributions which have been shown in Ref. [77] to arise for very large $M_{H^{ \pm}}$.
} 


$$
\begin{aligned}
& -\frac{1}{6}\left(c_{\beta} \mathcal{R}_{i 1}-s_{\beta} \mathcal{R}_{i 2}\right) \\
& \left.\times\left(\left(2 c_{\theta_{W}}^{2}+1\right) U_{j 1}^{\tilde{b} *} U_{k 1}^{\tilde{b}}+2 s_{\theta_{W}}^{2} U_{j 2}^{\tilde{b} *} U_{k 2}^{\tilde{b}}\right)\right],
\end{aligned}
$$

with

$$
\begin{aligned}
F_{1}= & A_{t}^{*} e^{-i \varphi_{u}}\left(\mathcal{R}_{i 2}-i c_{\beta} \mathcal{R}_{i 4}\right)-\mu_{\mathrm{eff}}\left(\mathcal{R}_{i 1}+i s_{\beta} \mathcal{R}_{i 4}\right) \\
& -\frac{\lambda v c_{\beta}\left(\mathcal{R}_{i 3}+i \mathcal{R}_{i 5}\right) e^{i \varphi_{s}}}{\sqrt{2}} \\
F_{2}= & A_{b}^{*}\left(\mathcal{R}_{i 1}-i s_{\beta} \mathcal{R}_{i 4}\right)-\mu_{\mathrm{eff}} e^{i \varphi_{u}}\left(\mathcal{R}_{i 2}+i c_{\beta} \mathcal{R}_{i 4}\right) \\
& -\frac{\lambda v s_{\beta}\left(\mathcal{R}_{i 3}+i \mathcal{R}_{i 5}\right) e^{i\left(\varphi_{s}+\varphi_{u}\right)}}{\sqrt{2}} .
\end{aligned}
$$

The one-loop QCD and EW contributions to the decay width are given by the sum of the virtual and real contributions, respectively,

$$
\begin{aligned}
\Gamma_{\mathrm{QCD} / \mathrm{EW}}^{(1)}\left(H_{i} \rightarrow \tilde{q}_{j} \tilde{q}_{k}^{*}\right)= & \Gamma_{\mathrm{QCD} / \mathrm{EW}}^{\mathrm{virt}}\left(H_{i} \rightarrow \tilde{q}_{j} \tilde{q}_{k}^{*}\right) \\
& +\Gamma_{\mathrm{QCD} / \mathrm{EW}}^{\mathrm{real}}\left(H_{i} \rightarrow \tilde{q}_{j} \tilde{q}_{k}^{*} g / \gamma\right) .
\end{aligned}
$$

For the virtual QCD contribution we have

$$
\begin{aligned}
& \Gamma_{\mathrm{QCD}}^{\mathrm{virt}}\left(H_{i} \rightarrow \tilde{q}_{j} \tilde{q}_{k}^{*}\right) \\
& =N_{F} R_{2}\left(M_{H_{i}}^{2}, M_{\tilde{q}_{j}}^{2}, M_{\tilde{q}_{k}}^{2}\right) \\
& \quad \times 2 \operatorname{Re}\left[\mathcal{M}_{H_{i} \tilde{q}_{j} \tilde{q}_{k}^{*}}^{0 *}\left(\sum_{i^{\prime}=1}^{5} \mathbf{Z}_{i i^{\prime}}^{H}\left(\mathcal{M}_{h_{i^{\prime}} \tilde{q}_{j} \tilde{q}_{k}^{*}}^{\Delta, \mathrm{QCD}}+\mathcal{M}_{h_{i^{\prime}} \tilde{q}_{j} \tilde{q}_{k}^{*}}^{\mathrm{CT}, \mathrm{QCD}}\right)\right)\right],
\end{aligned}
$$

with the 2-body phase space factor $R_{2}$ defined in Eq. (4.75). The expression for the virtual EW contribution is different from the QCD one due to an extra contribution containing the transition $h_{i} \rightarrow G, Z$. Explicitly, we have

$$
\begin{aligned}
& \Gamma_{\mathrm{EW}}^{\mathrm{virt}}\left(H_{i} \rightarrow \tilde{q}_{j} \tilde{q}_{k}^{*}\right) \\
& =N_{F} R_{2}\left(M_{H_{i}}^{2}, M_{\tilde{q}_{j}}^{2}, M_{\tilde{q}_{k}}^{2}\right) 2 \\
& \quad \times \operatorname{Re}\left[\mathcal{M}_{H_{i} \tilde{q}_{j} \tilde{q}_{k}^{*}}^{0 *} \sum_{i^{\prime}=1}^{5} \mathbf{Z}_{i i^{\prime}}^{H}\left(\mathcal{M}_{h_{i^{\prime}} \tilde{q}_{j} \tilde{q}_{k}^{*}}^{\Delta, \mathrm{EW}}+\mathcal{M}_{h_{i^{\prime}} \tilde{q}_{j} \tilde{q}_{k}^{*}}^{\mathrm{CT}, \mathrm{EW}}+\mathcal{M}_{h_{i^{\prime}} \tilde{q}_{j}}^{G Z, \operatorname{mix} \tilde{q}_{k}^{*}}\right)\right] .
\end{aligned}
$$

The explicit expressions for the counterterm contributions are quite lengthy and given in "Appendix A". We do not display, however, the more cumbersome amplitudes of the virtual QCD and EW contributions, $\mathcal{M}_{h_{i^{\prime}} \tilde{q}_{j} \tilde{q}_{k}^{*}}^{\Delta, \mathrm{QCD}}$ and $\mathcal{M}_{h_{i^{\prime}} \tilde{q}_{j} \tilde{q}_{k}^{*}}^{\Delta, \mathrm{EW}}$, respectively.
The real photon radiation contribution in the EW corrections is expressed in terms of the Bremsstrahlung integrals as

$$
\begin{aligned}
& \Gamma_{\mathrm{EW}}^{\mathrm{real}}\left(H_{i} \rightarrow \tilde{q}_{j} \tilde{q}_{k} \gamma\right) \\
& =\frac{N_{F}}{4 \pi^{2} M_{H_{i}}} Q_{q}^{2} \alpha \\
& \quad \times\left(-I_{1}-I_{2}-M_{\tilde{q}_{j}}^{2} I_{11}-M_{\tilde{q}_{k}}^{2} I_{22}+\left(M_{H_{i}}^{2}-M_{\tilde{q}_{j}}^{2}-M_{\tilde{q}_{k}}^{2}\right) I_{12}\right) \\
& \quad \times\left|\mathcal{M}_{H_{i} \tilde{q}_{j} \tilde{q}_{k}^{*}}^{0}\right|^{2} .
\end{aligned}
$$

As usual, we have neglected the arguments of the Bremsstrahlung integrals $I_{l}\left(M_{H_{i}}^{2}, M_{\tilde{q}_{j}}^{2}, M_{\tilde{q}_{k}}^{2}\right)$ and $I_{l m}$ $\left(M_{H_{i}}^{2}, M_{\tilde{q}_{j}}^{2}, M_{\tilde{q}_{k}}^{2}\right)(l, m=1,2$ and $j, k=1,2)$. The real gluon radiation contribution in the QCD corrections can be obtained from the EW real photon radiation contribution by replacing $Q_{q}^{2} \alpha$ with $C_{F} \alpha_{s}^{2}$, where $C_{F}=4 / 3$ for $S U(3)_{C}$. We have checked the UV and IR finiteness of the EW and QCD corrections. We have compared numerically with the NLO EW and QCD corrections in the OS scheme for the decay $A_{2} \rightarrow \tilde{t}_{1} \tilde{t}_{2}[6]$ using their description in the real NMSSM and found full agreement.

\section{Numerical results}

To illustrate the importance of the higher-order corrections to the decays of the light and heavy neutral Higgs bosons and to test the stability of the NLO results in various regions of the parameter space we have performed a scan in the NMSSM parameter space. The parameter points are checked against compatibility with the experimental constraints from the Higgs data by using the programs HiggsBounds5.3.2 [7880] and Higgs Signals2.2.3 [81]. These programs require as input the effective couplings of the Higgs bosons, normalized to the corresponding SM values, as well as the masses, the widths and the branching ratios of the Higgs bosons. These have been obtained for the SM and NMSSM Higgs bosons from the Fortran code NMSSMCALCEW $[14,82]$. One of the neutral CP-even Higgs bosons is identified with the SM-like Higgs boson - it will be called $h$ from now on - and its mass is required to lie in the range

$$
123 \mathrm{GeV} \leq m_{h} \leq 127 \mathrm{GeV} .
$$

For the SM input parameters we use the following values $[83,84]$

$$
\begin{aligned}
\alpha\left(M_{Z}\right) & =1 / 127.955, \quad \alpha_{s}^{\overline{\mathrm{MS}}}\left(M_{Z}\right)=0.1181 \\
M_{Z} & =91.1876 \mathrm{GeV} \quad M_{W}=80.379 \mathrm{GeV} \\
m_{t} & =172.74 \mathrm{GeV} \quad m_{b}^{\overline{\mathrm{MS}}}\left(m_{b}^{\overline{\mathrm{MS}}}\right)=4.18 \mathrm{GeV} \\
m_{c} & =1.274 \mathrm{GeV} \quad m_{s}=95.0 \mathrm{MeV} \\
m_{u} & =2.2 \mathrm{MeV} \quad m_{d}=4.7 \mathrm{MeV}
\end{aligned}
$$




$$
\begin{aligned}
& m_{\tau}=1.77682 \mathrm{GeV} \quad m_{\mu}=105.6584 \mathrm{MeV} \\
& m_{e}=510.9989 \mathrm{keV} \quad G_{F}=1.16637 \cdot 10^{-5} \mathrm{GeV}^{-2} .
\end{aligned}
$$

Concerning the NMSSM sector, we follow the SUSY Les Houches Accord (SLHA) format [85] in which the soft SUSY breaking masses and trilinear couplings are understood as $\overline{\mathrm{DR}}$ parameters at the scale

$\mu_{R}=M_{s}=\sqrt{m_{\tilde{Q}_{3}} m_{\tilde{t}_{R}}}$.

This is also the renormalization scale that we use in the computation of the higher-order corrections. Note that we chose the charged Higgs boson mass as an OS input parameter. The computation of the $\mathcal{O}\left(\alpha_{t} \alpha_{s}+\alpha_{t}^{2}\right)$ corrections to the Higgs boson masses is done in the $\overline{\mathrm{DR}}$ renormalization scheme of the top/stop sector. We have included the contribution of the gauge parameters $g_{1}, g_{2}$ into the conversion from pole to $\overline{\mathrm{DR}}$ top masses. In Table 1 we summarize the ranges applied in our parameter scan. In order to ensure perturbativity we apply the rough constraint

$\lambda^{2}+\kappa^{2}<0.7^{2}$.

The remaining mass parameters of the third generation sfermions that are not listed in the table are chosen as

$A_{b}=A_{\tau}=2 \mathrm{TeV}$, and $m_{\tilde{\tau}_{R}}=m_{\tilde{L}_{3}}=m_{\tilde{b}_{R}}=3 \mathrm{TeV}(5.5)$

The mass parameters of the first and second generation sfermions are set to

$m_{\tilde{u}_{R}, \tilde{c}_{R}}=m_{\tilde{d}_{R}, \tilde{s}_{R}}=m_{\tilde{Q}_{1,2}}=m_{\tilde{L}_{1,2}}=m_{\tilde{e}_{R}, \tilde{\mu}_{R}}=3 \mathrm{TeV}$.

We have performed two scans. In the first (smaller) scan we took care to select only such scenarios where the lightest CP-even Higgs boson $H_{1}$ is singlet-like and the second lightest CP-even Higgs boson is the SM-like Higgs boson. We refer to this scan as scanl in the following. In the second (larger) scan, called scan 2 in the following, we only retained scenarios where the SM-like Higgs boson is the lightest CPeven Higgs boson. Both scans allow for points that have a $\chi^{2}$ computed by HiggsSignals-2.2.3 that is consistent with an SM $\chi^{2}$ within $2 \sigma$. All the branching ratios shown in the following have been calculated by implementing the here presented higher-order corrections to the various decay widths in NMSSMCALCEW. In this way the new EW corrections are combined with the state-of-the-art higher-order QCD corrections already implemented in NMSSMCALC. Note, however, that the EW corrections are only taken into account if the respective decay is kinematically allowed. Otherwise, the corresponding decay width without the higher-order corrections discussed in this paper, which only apply for on-shell decays, are taken into account in the computation of the total decay width and branching ratios.

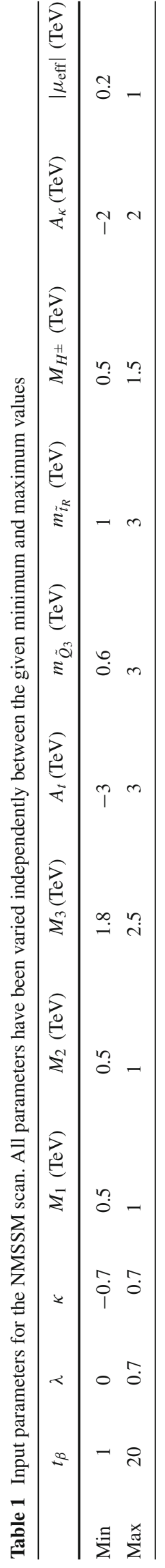




\subsection{Decays into SM fermion pairs}

In the old implementation in NMSSMCALC the tree-level couplings entering the various decay widths were improved by including loop effects in the Higgs mixing matrix elements. Thus, the tree-level rotation matrix $\mathcal{R}$ was replaced by the loop-corrected rotation matrix

$\mathcal{R}^{l}=\mathbf{Z}^{H} \mathcal{R}$,

evaluated at zero external momentum both at one-loop and at two-loop order to ensure unitarity. ${ }^{32}$ The implementation here differs by the fact that in the computation of $\mathbf{Z}^{H}$ we include the momentum dependence at one-loop order and we do not apply the approximation of Ref. [15] to deduce $\mathbf{Z}^{H}$ but proceed as described in Eqs. (3.84)-(3.86). In the following, we call the couplings where we apply $\mathcal{R}^{l}$ as obtained from Eq. (5.7) with zero external momentum and by applying the approximation of Ref. [15] 'effective treelevel couplings' while those with $\mathbf{Z}^{H}$ calculated according to Eqs. (3.84)-(3.86) including the momentum dependence at one-loop order are denominated 'improved couplings'.

The decays into SM fermion pairs in the old implementation in NMSSMCALC were calculated using the loopcorrected rotation matrix, $\mathcal{R}^{1}$, evaluated at zero external momenta and by including the $\Delta_{b}$ corrections $^{33}$ into the effective tree-level couplings, as specified in Ref. [14]. The thus obtained 'effective couplings' are given by ${ }^{34}$

$\tilde{g}_{h_{j} q \bar{q}}^{\text {eff, } S / P}=\tilde{g}_{h_{j} q \bar{q}}^{S / P},$.

Beyond the $\Delta_{b}$ approximation no further SUSY-EW nor SUSY-QCD corrections were included. To quantify the difference between the branching ratio computed in this paper and the old implementation in NMSSMCALC we introduce the relative change in the branching ratio for the decay $H_{i} \rightarrow X_{j} X_{k}$ as

$$
\begin{aligned}
& \Delta_{\mathrm{BR}}\left(H_{i} X_{j} X_{k}\right) \\
& =\frac{\mathrm{BR}_{\mathbf{Z}^{H}}^{\mathrm{SEW}(+\mathrm{SQCD})}\left(H_{i} \rightarrow X_{j} X_{k}\right)-\mathrm{BR}_{\mathcal{R}^{l}}^{\mathrm{tree}}\left(H_{i} \rightarrow X_{j} X_{k}\right)}{\max \left(\mathrm{BR}_{\mathbf{Z}^{H}}^{\mathrm{SEW}(+\mathrm{SQCD})}\left(H_{i} \rightarrow X_{j} X_{k}\right), \mathrm{BR}_{\mathcal{R}^{l}}^{\text {tree }}\left(H_{i} \rightarrow X_{j} X_{k}\right)\right)},
\end{aligned}
$$

with $X_{j} X_{k} \equiv f \bar{f}$ for the decays into fermions. Here the branching ratio $\mathrm{BR}_{\mathbf{Z}^{H}}^{\mathrm{SEW}(+\mathrm{SQCD})}\left(H_{i} \rightarrow f \bar{f}\right)$ means that we

\footnotetext{
32 We remind the reader, that in contrast the one-loop corrected masses are obtained at non-vanishing external momenta and the two-loop corrections at zero external momenta.

33 For simplicity, we collectively call them $\Delta_{b}$ corrections although we also include the corresponding corrections in the decays into strange quarks and into leptons.

34 We call them 'effective couplings' and not 'effective tree-level couplings' as they also contain the $\Delta_{b}$ corrections.
}

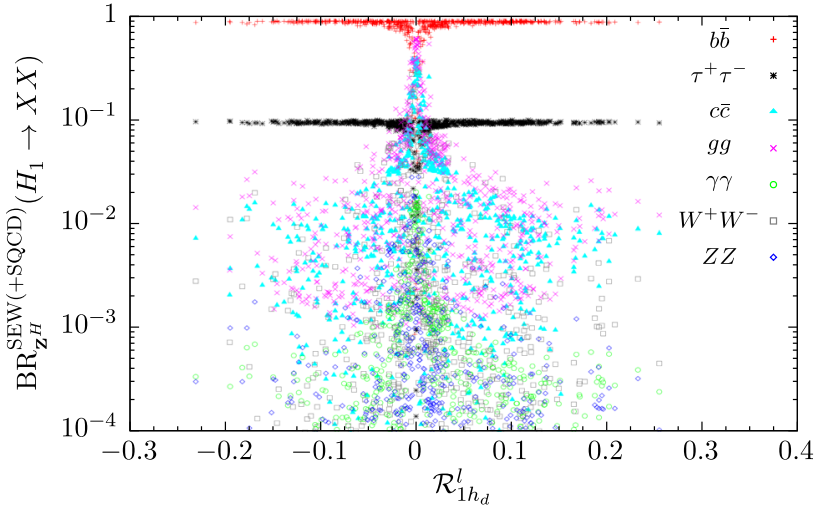

Fig. 1 Scan1: Loop-corrected branching ratios of the lightest Higgs boson, which is the singlet-like state, into SM particles versus the element $\mathcal{R}_{1 h_{d}}^{l}$ of the loop-corrected Higgs rotation matrix

include the SUSY-EW corrections (and SUSY-QCD corrections for the decays into quarks) together with the wavefunction renormalization factor into the decay width of the decay $H_{i} \rightarrow f \bar{f}$. The formulae are given by Eq. (4.12) for the decays into quarks and by Eq. (4.38) for the decays into leptons together with the definitions Eqs. (4.13) and (4.14). The branching ratio in the old implementation in NMSSMCALC is denoted by $\mathrm{BR}_{\mathcal{R}^{l}}^{\text {tree }}\left(H_{i} \rightarrow f \bar{f}\right)$ (although it also includes the $\Delta_{b}$ corrections where applicable). The quantity $\Delta_{\mathrm{BR}}$ hence gives information on the importance of the improvement of the branching ratios by the $\mathbf{Z}^{H}$ factor and the SEW(+SQCD) corrections. This quantity will also be used in the investigations of the decays into gauge boson pairs and into a pair of $Z$ and Higgs bosons.

The SM-like Higgs boson is given by the $h_{u}$-like Higgs state, ${ }^{35}$ and in our scans we found valid scenarios where this can be the lightest or the second lightest of the CP-even Higgs bosons. We first consider only the parameter points where the lightest CP-even Higgs boson $H_{1}$ is the singletlike state, i.e. has a large $h_{s}$ component. These are points obtained in the above described scanl. Here and in the following we denote a Higgs boson $H_{i}$ to be dominantly $x$-like ( $\left.x=h_{s}, h_{u}, h_{d}, a_{s}, a_{d}\right)$ if the corresponding matrix element squared $\left|\mathcal{R}_{i x}^{l}\right|^{2}$ exceeds $80 \%$. When $H_{1}$ is $h_{s}$-like, the question which final state has the largest decay width strongly depends on the amount of admixture of $h_{d}$ and $h_{u}$ components to the singlet-state. In Fig. 1 we show, for all parameter points that pass our constraints, the scatter plot of the $H_{1}$ branching ratios into SM particles against its $h_{d}$ component represented by the element $\mathcal{R}_{1 h_{d}}^{l}$ of the loop-corrected Higgs

\footnotetext{
35 As the SM-like Higgs boson has to comply with the experimentally measured Higgs rates and for small values of $\tan \beta$, as preferred by the NMSSM, is dominantly produced through gluon fusion it needs a substantial coupling to top quarks so that it is the $h_{u}$-dominated Higgs state that turns out to be SM-like.
} 
rotation matrix. ${ }^{36}$ The mass of $H_{1}$ lies between 70 and 118 $\mathrm{GeV}$ for these points. As can be inferred from the plot, the dominant decays are those into $b \bar{b}, c \bar{c}, \tau \bar{\tau}$ and $g g$. In most cases the branching ratio into a bottom-quark pair is dominant followed by the decay into $\tau \tau$. However, when the $h_{d}$ component of $H_{1}$ is very small the branching ratios into $g g$ and $c \bar{c}$ become competitive and can even be larger than those for the decay into $b \bar{b}$ with values beyond $60 \%$ for the $g g$ final state and of up to $35-39 \%$ for $c \bar{c}$ in some of the scenarios. In this case, i.e. for $\left|\mathcal{R}_{1 h_{d}}^{l}\right| \lesssim 0.02$, also the branching ratios into $\gamma \gamma$ and into the off-shell final state $W^{+*} W^{*-}$ increase and can reach up to about $30 \%$ in the latter and about $2 \%$ in the former case. The branching ratio into the off-shell $Z^{*} Z^{*}$ final state, which also increases then, is about one order of magnitude smaller than the one into $W^{+*} W^{-*}$. But already for $\left|\mathcal{R}_{1 h_{d}}^{l}\right| \gtrsim 0.02$ the decay into $b \bar{b}$ takes over again and reaches branching ratio values of up to $90 \%$ followed by the branching ratio into $\tau \tau$ with values of up to $10 \%$.

In order to investigate the importance of the higher-order corrections we define for our new implementation the relative correction of the partial width for the decay $H_{i} \rightarrow X_{j} X_{k}$ as

$$
\begin{aligned}
& \delta^{\mathrm{SEW}(+\mathrm{SQCD})}\left(H_{i} X_{j} X_{k}\right) \\
& =\frac{\Gamma_{\mathbf{Z}^{H}}^{\mathrm{SEW}(+\mathrm{SQCD})}\left(H_{i} \rightarrow X_{j} X_{k}\right)-\Gamma_{\mathbf{Z}^{H}}^{\text {tree }}\left(H_{i} \rightarrow X_{j} X_{k}\right)}{\Gamma_{\mathbf{Z}^{H}}^{\text {tree }}\left(H_{i} \rightarrow X_{j} X_{k}\right)},
\end{aligned}
$$

with the higher-order decay widths for the decays $H_{i} \rightarrow q \bar{q}$ into quarks given in Eq. (4.12) and the higher-order decay widths for the decays $H_{i} \rightarrow l \bar{l}$ into leptons given in Eq. (4.38) and with the tree-level decay width $\Gamma_{\mathbf{Z}^{H}}^{\text {tree including only the }}$ $\Delta_{b}$ corrections. The tree-level and higher-order decay widths are both evaluated with the new implementation of $\mathbf{Z}^{H}$. Note that the quantity $\delta$ gives information on the importance of the SEW $(+\mathrm{SQCD})$ corrections in the decay width alone as the factor $\mathbf{Z}^{H}$ cancels in the ratio. In Fig. 2 we show the scatter plot of the relative change of the branching ratios, $\Delta_{\mathrm{BR}}\left(H_{1} f \bar{f}\right), f=b, c, \tau$, for all the parameter points passing the constraints, against $\mathrm{BR}_{\mathbf{Z}^{H}}^{\mathrm{SEW}(+\mathrm{SQCD})}\left(H_{1} \rightarrow f \bar{f}\right)$.

The color code in Fig. 2 as well as in Figs. 3, 4, 5 and 6 denotes the sizes of the relative corrections of the partial decay widths. The points where the absolute value of $\delta^{\mathrm{SEW}(+\mathrm{SQCD})}$ exceeds $10 \%$ are marked in blue, those with $\left|\delta^{\mathrm{SEW}(+\mathrm{SQCD})}\right|$ in the $[5,10] \%$ range in black and those with relative corrections less than 5\% in red. For Figs. 5b and 6b we distinguish two regimes for the larger corrections, in blue where $\left|\delta^{\mathrm{SEW}+\mathrm{SQCD}}\right|$ is in the $[10,20] \%$ range and in green

\footnotetext{
$\overline{36}$ Note, that $\mathcal{R}_{1 h_{d}}^{l}$ is the $\left(1, h_{d}\right)$-component of the mixing matrix $\mathcal{R}^{l}$ given by Eq. (5.7), evaluated at zero external momentum both at oneand at two-loop order and where for the computation of $\mathbf{Z}^{H}$ the approximation of Ref. [15] is used. In the computation of the loop-corrected branching ratios, however, we of course use the new implementation described at the beginning of this section.
}

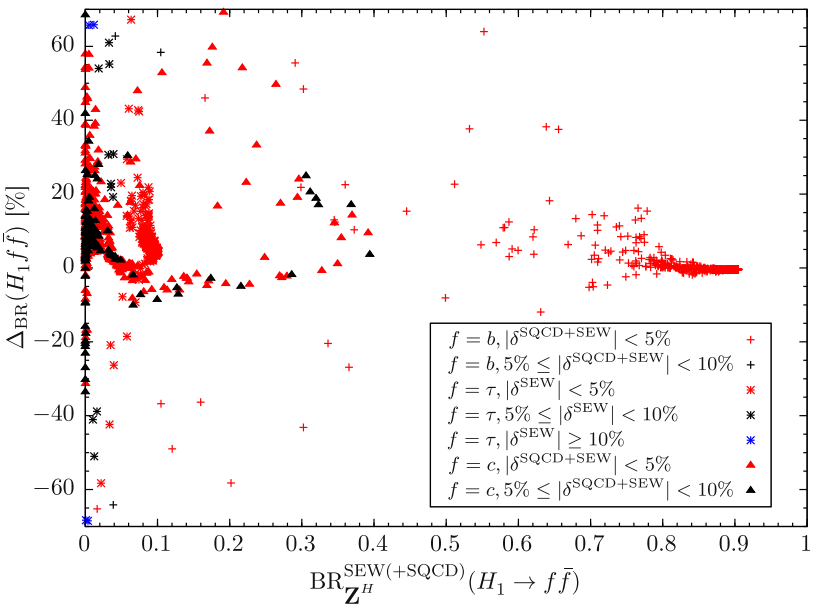

Fig. 2 Scan1: Relative difference $\Delta_{\mathrm{BR}}$ (see text, for definition) in percent for the $H_{1}$ decays into $b \bar{b}, \tau \bar{\tau}, c \bar{c}$. Red: relative corrections $\delta$ (see text, for definition) in percent of the SUSY-EW(+SUSY-QCD) corrections to the decay widths with $|\delta|<5 \%$; black: $5 \leq|\delta|<10 \%$; blue: $|\delta| \geq 10 \%$

where $\left|\delta^{\mathrm{SEW}+\mathrm{SQCD}}\right|$ is in the $[20,40] \%$ range; in Fig. $4 \mathrm{~b}$ we also add two other categories of points, in cyan where $\left|\delta^{\mathrm{SEW}+\mathrm{SQCD}}\right|$ is in the $[40,60] \%$ range and in pink where $\left|\delta^{\mathrm{SEW}+\mathrm{SQCD}}\right|$ is in the $[60,80] \%$ range. Note that for the $\tau$ decays there are no SUSY-QCD corrections. The ballpark of the relative change $\Delta_{\mathrm{BR}}\left(H_{1} b \bar{b}\right)$ in the branching ratios between the old and the new implementation ranges below about $30 \%$ with vertex corrections $|\delta|$ smaller than $5 \%$. There are some very rare scenarios where $\left|\Delta\left(H_{1} b \bar{b}\right)\right|$ exceeds $50 \%$ and where at the same time the relative vertex corrections are between 5 and $10 \%$. We investigated these cases and observed that there is an accidental cancellation either in the effective tree-level or in the improved couplings. These parameter points lead to similar results for the $\tau$ final states, i.e. $\left|\Delta_{\mathrm{BR}}\left(H_{1} \tau \bar{\tau}\right)\right|>50 \%$ and at the same time $|\delta|$ between 5 and $10 \%$. The cancellation results in a suppression of the branching ratio, to less than $4 \%$ for the $\tau \bar{\tau}$ final state and $10 \%$ at maximum for the $b \bar{b}$ final state. In most of the cases, the large $\Delta_{\mathrm{BR}}\left(H_{1} f \bar{f}\right)$ is due to the use of the wave-function renormalization factor $\mathbf{Z}^{H}$, however. There are also cases with a cancellation between the SUSY-EW/SUSY-QCD corrections and the wave-function renormalization factor $\mathbf{Z}^{H}$ correction. This results in $\Delta_{\mathrm{BR}}\left(H_{1} c \bar{c}\right)$ being less than $1 \%$.

We have performed the same analysis for the heavier Higgs bosons, using the full set of points from our scan2. Figure 3 is the scatter plot of the $\Delta_{\mathrm{BR}}(h f \bar{f}), f=b, \tau, c, t$, against $\mathrm{BR}_{\mathbf{Z}^{H}}^{\mathrm{SEW}(+\mathrm{SQCD})}(h \rightarrow f \bar{f})$ for an SM-like Higgs boson $h$, while Figs. 4, 5, and 6 are the scatter plots of $\Delta_{\mathrm{BR}}\left(H_{i} f \bar{f}\right), f=b, t$, against $\mathrm{BR}_{\mathbf{Z}^{H}}^{\mathrm{SEW}(+\mathrm{SQCD})}\left(H_{i} \rightarrow f \bar{f}\right)$ for a heavy $a_{s^{-}}, a$ - and $h_{d}$-like Higgs boson i.e. $H_{i}=H_{a_{s}}$, $H_{a}, H_{h_{d}}$, respectively. As before, the SM-like Higgs boson $h$ is always $h_{u}$-like and decays dominantly into a bottom- 


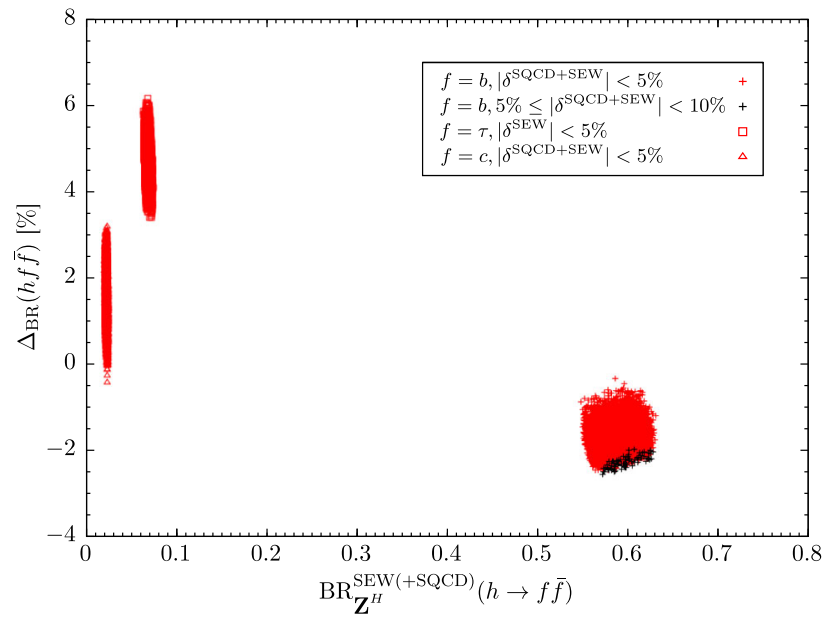

Fig. 3 Scan2: Same as Fig. 2 but for the SM-like Higgs boson $h$ that is $h_{u}$-like

quark pair with a branching ratio of about $60 \%$, as expected, followed by the decay into a $\tau$ pair and the decay into a $c$-quark pair. As can be inferred from Fig. 3, the relative changes between the old and the new implementation are much smaller than for the singlet-like lightest Higgs boson and amount only to a few percent. The relative vertex corrections $|\delta|$ are below $10 \%$ for the $b$-quark pair final state and below $5 \%$ for the decays both into $\tau \bar{\tau}$ and $c \bar{c}$.

For the heavy Higgs bosons, the decay into a top quark pair can become kinematically possible. We start by discussing the decay pattern of the heavy singlet state $H_{a_{s}}$, with a mass between $120 \mathrm{GeV}$ and $1.7 \mathrm{TeV}$, into the $b$-quark and $t$-quark final states, presented in Fig. $4 .{ }^{37}$ For the $b \bar{b}$ final states the relative change in the branching ratios due to the new implementation is mostly between -20 and $20 \%$. We also find points where the relative change is close to $100 \%$, in particular for branching ratios close to $100 \%$. Most points exhibit small relative vertex corrections (see, red points in Fig. 4a), so that the large changes of $\Delta_{\mathrm{BR}}\left(H_{a_{s}} b \bar{b}\right)$ are due to the implementation of $\mathbf{Z}^{H}$. This is especially the case for large branching ratios close to $100 \%$. There are a few points where the relative vertex corrections lie between 5 and 10\% (black) and even above $10 \%$ (blue). This happens for the cases where the effective tree-level couplings $H_{a_{s}} f \bar{f}$ are suppressed. The relative change $\Delta_{\mathrm{BR}}$ can still be very small when the effects from the $\mathbf{Z}^{H}$ factor and the vertex corrections cancel. The decay pattern for the $t \bar{t}$ channel, finally, is displayed in Fig. 4b. The branching ratio takes all values between almost 0 and $100 \%$. The relative changes $\Delta_{\mathrm{BR}}\left(H_{a_{s}} t \bar{t}\right)$ are mostly between -20 and $20 \%$ and close to $0 \%$ for large branching ratios above about $60 \%$. We also observe large $\Delta_{\mathrm{BR}}$, in particular for branching ratios close to zero. This is mainly due to very

\footnotetext{
37 Since we will not gain much new information, for $H_{a_{s}}, H_{a}$ and $H_{h_{d}}$ we do not show the corresponding plots into $\tau \bar{\tau}$ and $c \bar{c}$.
}

suppressed effective tree-level $H_{a_{s}} t \bar{t}$ couplings corresponding to the regions where the branching ratio $\operatorname{BR}\left(H_{a_{s}} \rightarrow b \bar{b}\right)$ is enhanced. These regions correspond to large values of $\tan \beta$ close to the upper bound in our scan, or to smaller mass values of $H_{a_{s}}$ with not sufficient phase space to decay into an on-shell top-quark pair. In these regions the relative corrections $|\delta|$ are most of the time below $40 \%$, and for cases where $|\delta|<5 \%$ the large changes in $\Delta_{\mathrm{BR}}$ are mostly due to the use of the wave-function renormalization factor $\mathbf{Z}^{H}$. For larger branching ratios the relative corrections $|\delta|$ are mostly below $10 \%$ (red and black points). Some rare scenarios display corrections above $40 \%$ and up to $80 \%$ (in cyan and in pink), again mostly in regions with lower branching ratios.

Similar observations can be made for the other heavier Higgs states $H_{a}$ (with a mass between $539 \mathrm{GeV}$ and $2 \mathrm{TeV}$ ) and $H_{h_{d}}$ (with a mass between $548 \mathrm{GeV}$ and $2 \mathrm{TeV}$ ), with the notable exception that the relative changes $\Delta_{\mathrm{BR}}\left(H_{a / h_{d}} X_{i} X_{j}\right)$ are more reduced and never reach $100 \%$. The relative changes $\Delta_{\mathrm{BR}}\left(H_{a / h_{d}} b \bar{b}\right)$ are most of the time positive and below $40 \%$ as seen in Figs. 5a and 6a. The decays into top-quark pairs can be dominant where the decays into $b \bar{b}$ are suppressed, and the relative changes $\Delta_{\mathrm{BR}}$ between the old and new implementation are close to zero when $\operatorname{BR}\left(H_{a / h_{d}} \rightarrow t \bar{t}\right) \rightarrow 100 \%$ as seen in Figs. $5 \mathrm{~b}$ and $6 \mathrm{~b}$. For some rare scenarios the relative vertex corrections $|\delta|$ can reach $40 \%$, depicted in green in the figures. Note that $\operatorname{BR}\left(H_{a / h_{d}} \rightarrow b \bar{b}\right)$ can reach $90 \%$, corresponding to regions where the effective tree-level coupling $H_{a / h_{d}} b \bar{b}$ is strongly enhanced due to large values of $\tan \beta$ while at the same time the effective tree-level coupling $H_{a / h_{d}} t \bar{t}$ is strongly suppressed.

\subsection{Decays into a massive gauge boson pair}

In the CP-conserving case, the heavy Higgs boson that can decay into two on-shell massive gauge bosons is $h_{d}$-like. The tree-level coupling of a Higgs boson $H_{i}$ to $V V(V=W, Z)$ is proportional to

$\mathcal{R}_{i, 1} c_{\beta}+\mathcal{R}_{i, 2} s_{\beta}$.

Due to the SM-like (i.e. $h_{u}$-like) Higgs boson coupling with almost SM-strength to the massive gauge bosons the treelevel coupling of the $h_{d}$-like heavy Higgs boson to $V V$ is almost zero because of sum rules. This leads to very suppressed tree-level partial decay widths $\Gamma\left(H_{h_{d}} \rightarrow V V\right)$.

In order to compare the results obtained in this paper with the old implementation in NMSSMCALC using the tree-level coupling together with the loop-corrected rotation matrix $\mathcal{R}^{l}$, we show in Fig. $7 \mathrm{a}$ the relative change $\Delta_{\mathrm{BR}}\left(H_{h_{d}} W W\right)$ of the branching ratio into $W W$ between the old and the new implementation including the NLO-EW vertex corrections as described in Sect. 4.2 and the improvement with the $\mathbf{Z}^{H}$ factor, as a function of the loop-corrected branching ratio 


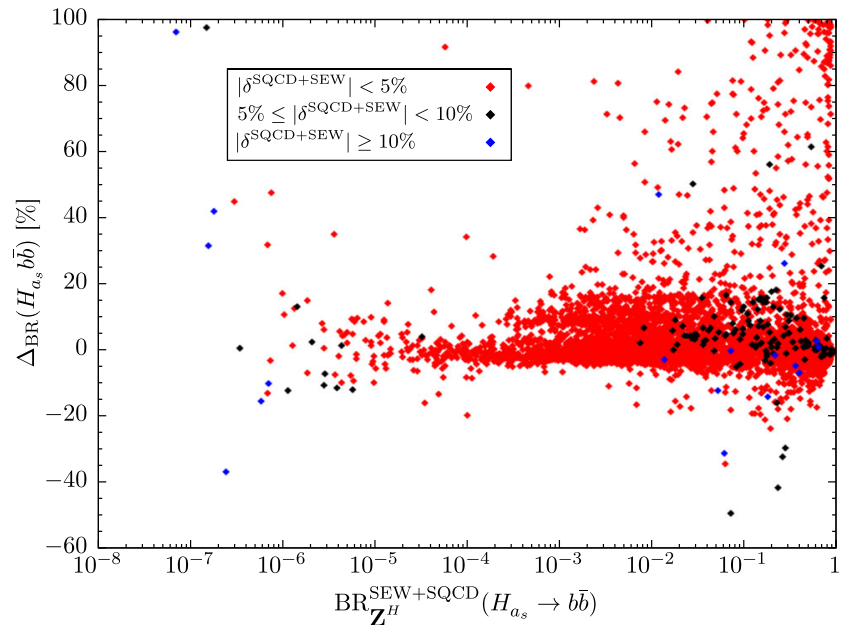

(a)

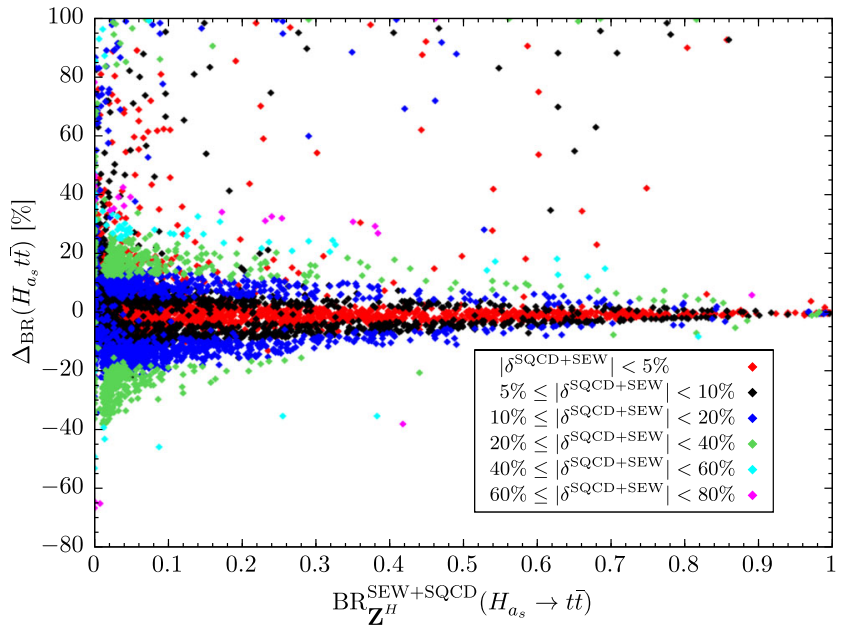

(b)

Fig. 4 Scan2: Same as Fig. 2 but for the heavier $a_{s}$-like Higgs boson $H_{a_{s}}$ decaying into $b \bar{b}$ (left) and $t \bar{t}$ (right)

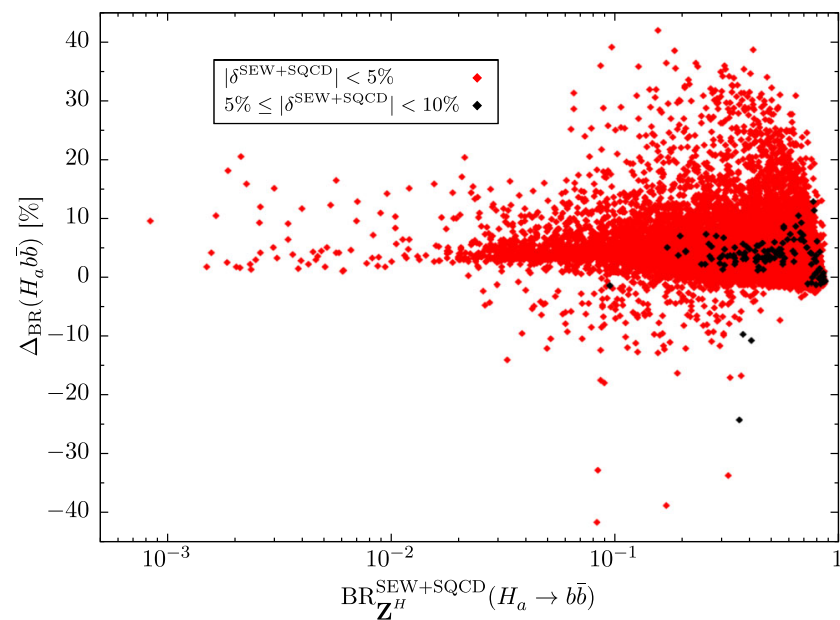

(a)

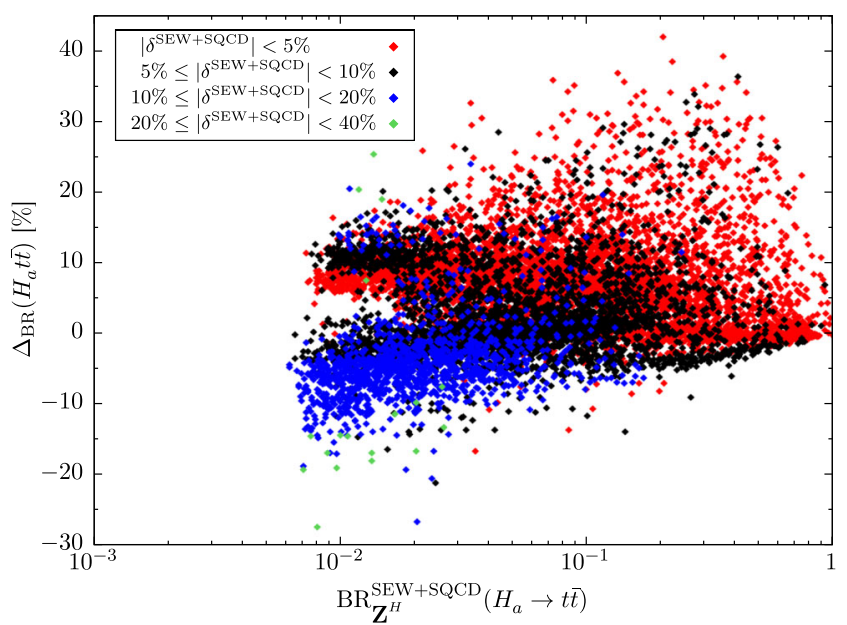

(b)

Fig. 5 Scan2: Same as Fig. 2 but for the heavier $a$-like Higgs boson $H_{a}$ decaying into $b \bar{b}$ (left) and $t \bar{t}$ (right)

$\mathrm{BR}_{\mathbf{Z}^{H}}^{\mathrm{SEW}}\left(H_{h_{d}} \rightarrow W^{+} W^{-}\right)$. The plotted points are those of our scan that pass the constraints we have applied. We display in Fig. $7 \mathrm{~b}$ the same but for the decay into $Z Z$. The color and symbol code denotes the magnitude of the relative NLO electroweak vertex corrections alone, with $\delta$ defined in Eq. (5.10). As can be inferred from the plots both the $\mathbf{Z}^{H}$ factor and the NLO electroweak corrections can be responsible for the large relative changes in the branching ratios. This is in particular reflected by the black points for which the vertex corrections are below $20 \%$ and at the same time the relative changes $\Delta_{\mathrm{BR}}$ can reach up to $100 \%$. In some cases there is a cancellation between the two contributions (the vertex corrections and the $\mathbf{Z}^{H}$ factor) leading to relatively small relative changes in the branching ratios. These cases are the pentagon-marked full (red) points in Fig. 7a for the decay $H_{h_{d}} \rightarrow W^{+} W^{-}$, which are mostly located in regions where
$\left|\Delta_{\mathrm{BR}}\left(H_{h_{d}} W W\right)\right| \lesssim 25 \%$ while the relative vertex correction $|\delta|$ is at least $100 \%$. In the case of the decay into a $Z$ boson pair, however, the bulk of these pentagon-marked full (red) points, indicating again a relative vertex correction $|\delta|$ of at least $100 \%$, induces large relative changes of $\Delta_{\mathrm{BR}}\left(H_{h_{d}} Z Z\right)$ close to $100 \%$. These points correspond to a region which is discussed in more detail in the next paragraph.

We also note that there are two regions concentrating many points for the decay into a $Z$ boson pair, the region for which $\Delta_{\mathrm{BR}}\left(H_{h_{d}} Z Z\right) \simeq 0 \%$ and the one for which $\Delta_{\mathrm{BR}}\left(H_{h_{d}} Z Z\right) \simeq$ $100 \%$. This is in contrast to the decay into a $W$ boson pair which is mostly centered around $\Delta_{\mathrm{BR}}\left(H_{h_{d}} W W\right) \simeq 0 \%$ for vertex corrections $|\delta|<80 \%$ and much more scattered for the points where $80<|\delta|<100 \%$, displayed with cyanpentagon-marked points (for $|\delta| \geq 100 \%$ the above described cancellation takes place in the decay $H_{h_{d}} \rightarrow W^{+} W^{-}$). This 


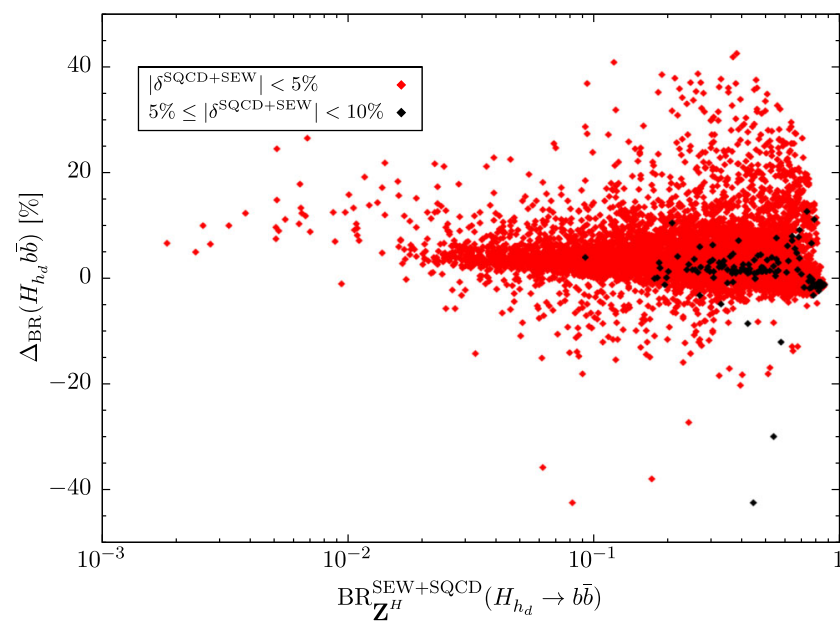

(a)

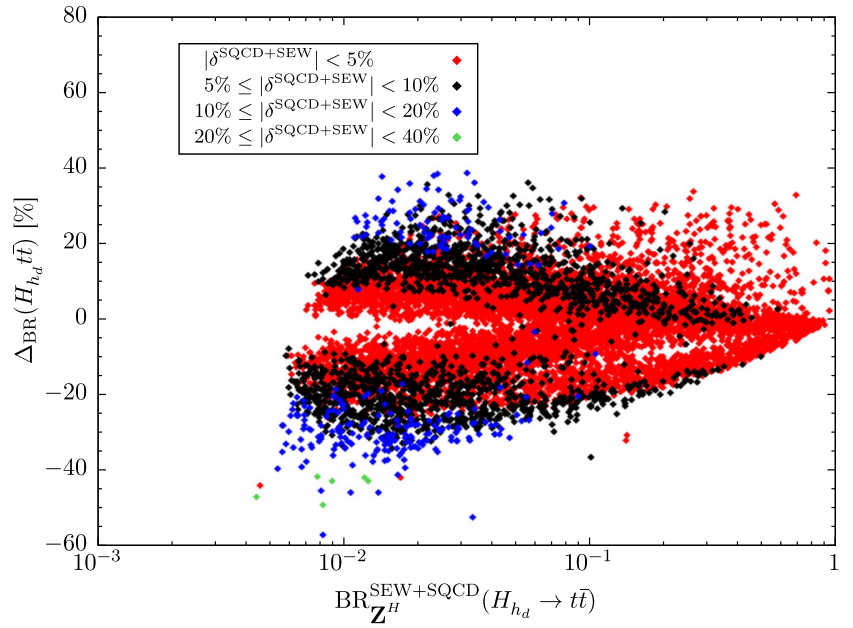

(b)

Fig. 6 Scan2: Same as Fig. 2 but for the heavier $h_{d}$-like Higgs boson $H_{h_{d}}$ decaying into $b \bar{b}$ (left) and $t \bar{t}$ (right)

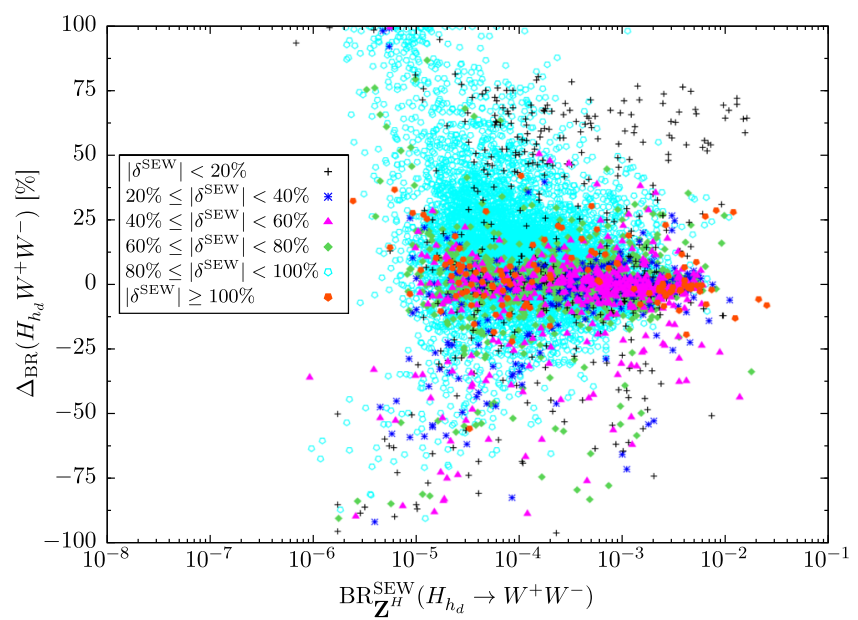

(a)

Fig. 7 Scan2: Relative difference $\Delta_{\mathrm{BR}}$ in percent for the $h_{d}$-like Higgs boson $H_{h_{d}}$ into a $W$ boson pair (left) and into a $Z$ boson pair (right) as a function of the corresponding loop-corrected branching ratio. Black: relative corrections $\delta^{\mathrm{SEW}}$ in percent of the SUSY-EW corrections to

presence of the second region in the $Z$ boson final state, for which $\Delta_{\mathrm{BR}}$ is close to $100 \%$ can be explained by the occurrence of many parameter points having a very suppressed tree-level coupling $H_{h_{d}} Z Z$. They also correspond to regions where the loop-corrected partial decay width $\Gamma\left(H_{h_{d}} \rightarrow Z Z\right)$ is higher, up to $1 \mathrm{GeV}$, while the decay width is at most $5 \mathrm{MeV}$ for the region centered around $\Delta_{\mathrm{BR}}\left(H_{h_{d}} Z Z\right)=0$. Note, that while the tree-level couplings $H_{h_{d}} Z Z$ and $H_{h_{d}} W W$ are the same, the loop-corrected decay widths differ by the fact that the decay into $W W$ bosons receives real corrections and that in the one-loop squared contributions to the decay width

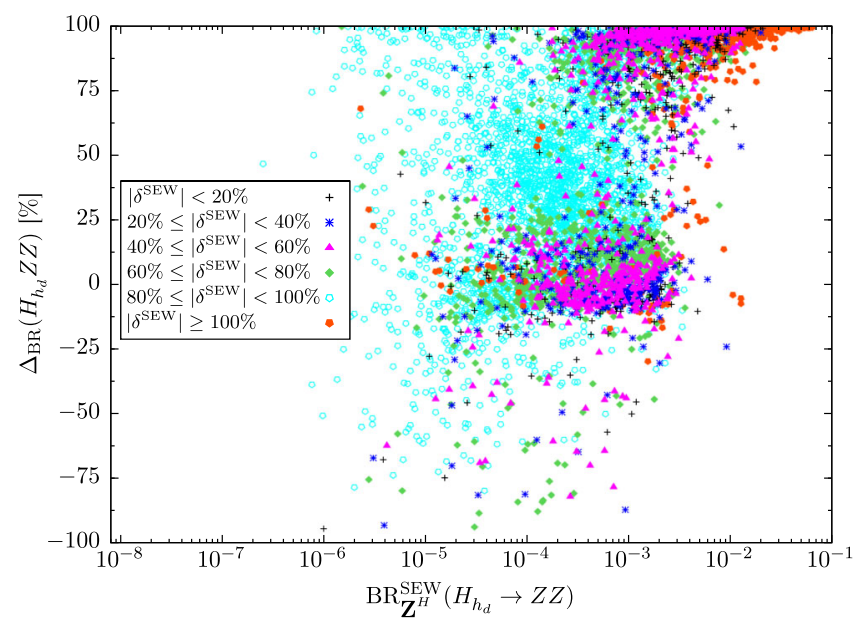

(b)

the decay widths with $\left|\delta^{\mathrm{SEW}}\right|<20 \%$; blue: $20 \leq\left|\delta^{\mathrm{SEW}}\right|<40 \%$; pink: $40 \leq\left|\delta^{\mathrm{SEW}}\right|<60 \%$; green: $60 \leq\left|\delta^{\mathrm{SEW}}\right|<80 \%$; cyan: $80 \leq\left|\delta^{\mathrm{SEW}}\right|<100 \%$; red: $\left|\delta^{\mathrm{SEW}}\right| \geq 100 \%$

$H_{h_{d}} \rightarrow W^{+} W^{-}$we only include the (s)fermion contributions in contrast to the decay $H_{h_{d}} \rightarrow Z Z{ }^{38}$

\subsection{Decays into a $Z$ boson and a Higgs boson}

In the searches for heavy pseudoscalars, this decay can be an important search channel $[86,87]$. We are interested here in how large the branching ratio can be and how important are the newly included higher-order corrections, in the case

\footnotetext{
${ }^{38}$ We remind the reader that we take into account this part of the two-loop corrections in case the one-loop corrected partial decay width becomes negative, see also Eq. (4.62).
} 
for which on-shell decays are possible. With the obtained valid set of parameter points, we present in Fig. 8a scatter plots of the relative changes of the branching ratios between the old and new implementation for the decay of a heavy pseudoscalar-like Higgs boson $H_{a}$ into $Z H_{1}$ and in Fig. $8 \mathrm{~b}$ for the decay into $\mathrm{ZH}_{2}$, against the respective loop-corrected branching ratios. The color and marker codes denote the relative sizes of the one-loop vertex corrections within specific ranges, identical to the ranges used in the previous section for the decays into gauge boson pairs. The mass values of the individual involved CP-even Higgs bosons in the final state range in $123 \mathrm{GeV} \leq m_{H_{1}} \leq 127 \mathrm{GeV}$ and $463 \mathrm{GeV} \leq$ $m_{H_{2}} \leq 1.73 \mathrm{TeV}$, while the mass of the decaying Higgs boson ranges in $539 \mathrm{GeV} \leq m_{H_{a}} \leq 2.0 \mathrm{TeV}$ for the on-shell decay into $Z H_{1}$ pairs and in $713 \mathrm{GeV} \leq m_{H_{a}} \leq 2.0 \mathrm{TeV}$ for the on-shell decay into $\mathrm{ZH}_{2}$ pairs. As can already be inferred from the mass ranges, the $H_{1}$ state is the SM-like Higgs boson $h$, while the $\mathrm{H}_{2}$ state is the singlet-like scalar Higgs boson $H_{h_{s}}$.

We observe that the branching ratios into the $Z H_{1}$ final state remain very small, below $0.4 \%$, while those of the decay into $\mathrm{ZH}_{2}$ can reach $11 \%$. This is due to the nature of the $H_{1}$ Higgs boson that is SM-like, with very suppressed tree-level $H_{1} H_{a} Z$ couplings. The relative changes $\Delta_{\mathrm{BR}}$ of the branching ratio for the decay $H_{a} \rightarrow Z H_{1}$ are mostly between 0 and $-75 \%$ corresponding to relative vertex corrections $\left|\delta^{\mathrm{SEW}}\right|$ being at most $60 \%$ (black, blue, and pink points), while a few points corresponding to higher vertex corrections up to more than $100 \%$ (green, cyan, and red points) can reach $\Delta_{\mathrm{BR}}= \pm 100 \%$. These extreme points correspond to very small values for the branching ratios themselves which explains in turn the very large relative corrections $|\delta|$ that we observe. Note that in these decays we take into account the one-loop squared term as described in Eq. (4.68) which makes up for the main contribution to the very large relative corrections. As for the decay $H_{a} \rightarrow \mathrm{ZH}_{2}$ the relative correction $\Delta_{\mathrm{BR}}$ is most of the time between 0 and $\pm 25 \%$, corresponding to points where the relative vertex corrections $|\delta|$ are below $20 \%$. A few points display larger $\Delta_{\mathrm{BR}}$ values, and also larger relative vertex corrections $|\delta|$ that can reach $100 \%$ and even beyond, again for points that display very small branching ratios, below about $10^{-4} \%$. Note that there are points for which the correction $\left|\Delta_{\mathrm{BR}}\right|$ is rather limited, below $25 \%$, while the relative vertex correction can reach $40 \%$ (for one scenario even more than 100\%). This can be explained by a sign compensation between the $\mathbf{Z}^{H}$ factor and the vertex correction.

The corresponding results for the heavy singlet-like Higgs boson $H_{h_{s}}$ decaying into $Z A_{1}$ and $Z A_{2}$ are shown in Fig. 9a, $\mathrm{b}$, respectively. We see that the maximum achieved branching ratios for the decay $H_{h_{s}} \rightarrow Z A_{1}$ are below $20 \%$ and for a large number of parameter points are tiny. In the case of the decay $H_{h_{s}} \rightarrow Z A_{2}$ the branching ratio can reach around
$15 \%$. In most of the cases $A_{1}$ is singlet-like, corresponding to points where the branching ratio is small (below 10\%), while $A_{2}$ is doublet-like. The mass values of the individual involved Higgs bosons in the final state range in $120 \mathrm{GeV} \leq$ $m_{A_{1}} \leq 1.50 \mathrm{TeV}$ and $562 \mathrm{GeV} \leq m_{A_{2}} \leq 1.63 \mathrm{TeV}$, while the mass of the decaying Higgs boson ranges in $464 \mathrm{GeV} \leq$ $m_{H_{h_{s}}} \leq 1.75 \mathrm{TeV}$ for the on-shell decay into $Z A_{1}$ pairs and in $696 \mathrm{GeV} \leq m_{H_{h_{s}}} \leq 1.75 \mathrm{TeV}$ for the on-shell decay into $\mathrm{ZH}_{2}$ pairs. The cases with larger branching ratios for the decay $H_{h_{s}} \rightarrow Z A_{1}$ (larger than $10 \%$ ) correspond mostly to the few $A_{1}$ pseudoscalar Higgs bosons with doublet-like admixture and mass values above $400 \mathrm{GeV}$.

The relative changes $\Delta_{\mathrm{BR}}$ in the branching ratios are mostly positive, and can reach values of $100 \%$. For some very rare scenarios we get $\Delta_{\mathrm{BR}}\left(H_{h_{s}} Z A_{1}\right)$ close to $-100 \%$, while $\Delta_{\mathrm{BR}}\left(H_{h_{s}} Z A_{2}\right)$ is not below $-15 \%$. The relative vertex corrections are moderate for the decay $H_{h_{s}} \rightarrow Z A_{1}$, mostly $|\delta| \leq 20 \%$ (black points). This means that the large changes in $\Delta_{\mathrm{BR}}\left(H_{h_{s}} Z A_{1}\right)$ are mostly due to the $\mathbf{Z}^{H}$ factor. For very small branching fractions below $10^{-4} \%$ larger vertex corrections are possible, mainly because the denominator in the definition of $\delta$ is very small in these regions and can lead to sharp changes in $\delta$. Note that the bulk of the changes between the old and the new implementation in these cases stems from the vertex corrections. In the case of the decay $H_{h_{s}} \rightarrow Z A_{2}$ the relative vertex corrections are mostly small, with values $|\delta|<5 \%$ (red triangle-marked points). For large relative changes $\Delta_{\mathrm{BR}}\left(H_{h_{s}} Z A_{2}\right)$ the $\mathbf{Z}^{H}$ factor is responsible then.

\subsection{Decays into charginos and neutralinos}

We start by investigating the loop corrections to the masses of the charginos and neutralinos using the three renormalization schemes OS1, OS2 and $\overline{\mathrm{DR}}$, imposed on the two gaugino masses $M_{1}$ and $M_{2}$, as defined in Sect. 3.1.2. According to the SLHA format that we apply in our code, $M_{1}, M_{2}$ are $\overline{\mathrm{DR}}$ parameters given at the scale $M_{\mathrm{SUSY}}=\sqrt{m_{\tilde{Q}_{3}} m_{\tilde{t}_{R}}}$. When we use the OS schemes we have to translate the $\overline{\mathrm{DR}}$ input parameters to the OS values by applying the approximate transformation formulae

$$
\begin{aligned}
& M_{1}^{\mathrm{OSi}}=M_{1}^{\overline{\mathrm{DR}}}-\delta M_{1}^{\mathrm{finOSi}} \\
& M_{2}^{\mathrm{OSi}}=M_{2}^{\overline{\mathrm{DR}}}-\delta M_{2}^{\mathrm{finOSi}}
\end{aligned}
$$

where $\delta M_{1 / 2}^{\mathrm{finOSi}}$ are the finite parts of the $M_{1 / 2}$ counterterms computed in the OSi $(i=1,2)$ renormalization scheme. Since the finite parts $\delta M_{1 / 2}^{\text {finOSi }}$ should be computed with OS input parameters we have used an iterative method to obtain these. For all parameter points in our scan, the size of the loop corrections to the neutralino and charginos masses, quantified by $\Delta M_{i}^{\tilde{\chi}}=M_{\tilde{\chi}_{i}}^{\text {loop }}-m_{\tilde{\chi}_{i}}^{\text {tree }}$, with $i=1, \ldots, 5$ for the 


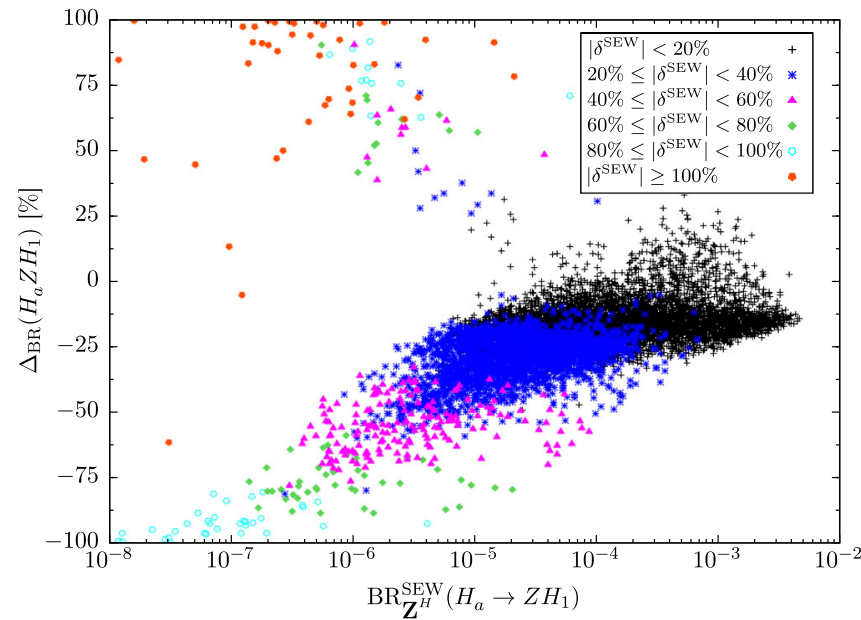

(a)

Fig. 8 Scan2: Relative difference $\Delta_{\mathrm{BR}}$ in percent for the $H_{a}$-like Higgs boson decay into $Z H_{1}$ (left) and into $Z H_{2}$ (right) as a function of the corresponding loop-corrected branching ratio. Black: relative corrections $\delta^{\mathrm{SEW}}$ in percent of the SUSY-EW corrections to the decay widths with

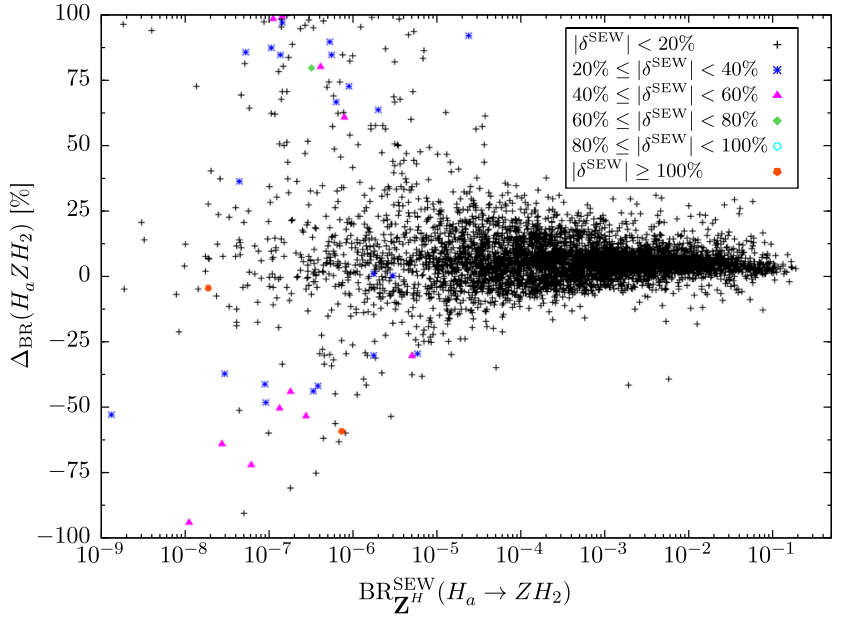

(b)

$\left|\delta^{\mathrm{SEW}}\right|<20 \%$; blue: $20 \leq\left|\delta^{\mathrm{SEW}}\right|<40 \%$; pink: $40 \leq\left|\delta^{\mathrm{SEW}}\right|<60 \%$; green: $60 \leq\left|\delta^{\mathrm{SEW}}\right|<80 \%$; cyan: $80 \leq\left|\delta^{\mathrm{SEW}}\right|<100 \%$; red: $\left|\delta^{\mathrm{SEW}}\right| \geq 100 \%$

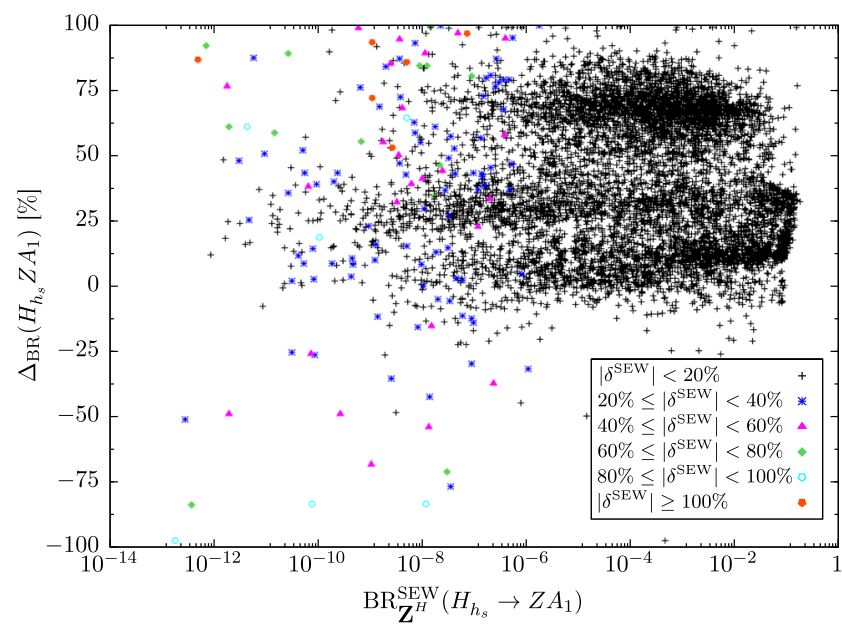

(a)

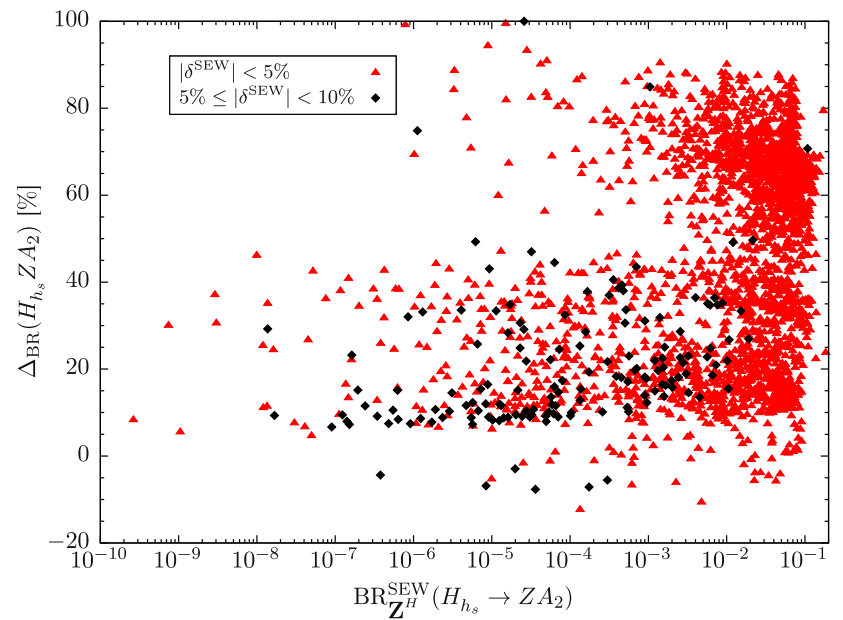

(b)

Fig. 9 Scan2: Same as in Fig. 8 but for a heavy singlet-like Higgs boson $H_{h_{s}}$ decaying into $Z A_{1}$ and $Z A_{2}$ pairs. In the right plot, however, red: $\left|\delta^{\mathrm{SEW}}\right|<5 \%$; black: $5 \leq\left|\delta^{\mathrm{SEW}}\right|<10 \%$

neutralinos and $i=1,2$ for the charginos, never exceeds 46 $\mathrm{GeV}$.

We illustratively present here a particular point, called scenariol, with the soft SUSY breaking masses and trilinear couplings given by

$$
\begin{aligned}
m_{\tilde{t}_{R}} & =1384 \mathrm{GeV}, m_{\tilde{Q}_{3}}=1743 \mathrm{GeV} \\
m_{\tilde{b}_{R}} & =m_{\tilde{L}_{3}}=m_{\tilde{\tau}_{R}}=3000 \mathrm{GeV} \\
\left|A_{u, c, t}\right| & =3594 \mathrm{GeV},\left|A_{d, s, b}\right|=2000 \mathrm{GeV}, \\
\left|A_{e, \mu, \tau}\right| & =2000 \mathrm{GeV}, \\
\left|M_{1}\right| & =560 \mathrm{GeV},\left|M_{2}\right|=684 \mathrm{GeV}
\end{aligned}
$$

$$
\begin{aligned}
\left|M_{3}\right| & =2494 \mathrm{GeV}, \\
\varphi_{A_{e, \mu, \tau}} & =\varphi_{A_{d, s, b}}=0, \varphi_{A_{u, c, t}}=\varphi_{M_{1}} \\
& =\varphi_{M_{2}}=\varphi_{M_{3}}=0,
\end{aligned}
$$

and the remaining input parameters set to ${ }^{39}$

$$
\begin{aligned}
|\lambda| & =0.307, \quad|\kappa|=0.517, \quad \operatorname{Re} A_{\kappa}=361 \mathrm{GeV}, \\
\left|\mu_{\mathrm{eff}}\right| & =272 \mathrm{GeV}, \\
\varphi_{\lambda} & =\varphi_{\kappa}=\varphi_{u}=0, \quad \varphi_{\mu_{\mathrm{eff}}}=\pi \\
\tan \beta & =9.38, \quad M_{H^{ \pm}}=1393 \mathrm{GeV}
\end{aligned}
$$

\footnotetext{
39 The imaginary part of $A_{\kappa}$ is obtained from the tadpole condition.
} 
Table 2 Scenario1: Masses and main components of the neutral Higgs bosons at two-loop order $\mathcal{O}\left(\alpha_{t} \alpha_{s}+\alpha_{t}^{2}\right)$, using $\overline{\mathrm{DR}}$ renormalization in the top/stop sector

Table 3 Scenario1: Masses and main components of the neutralinos and charginos at tree and one-loop level in the three renormalization schemes OS1, OS2 and $\overline{\mathrm{DR}}$

\begin{tabular}{llllll}
\hline$\overline{\mathrm{DR}}$ & $H_{1}$ & $H_{2}$ & $H_{3}$ & $H_{4}$ & $H_{5}$ \\
\hline Two-loop $\mathcal{O}\left(\alpha_{t} \alpha_{s}+\alpha_{t}^{2}\right)$ & 125.14 & 698.05 & 813.53 & 1391.6 & 1392.56 \\
Main component & $h_{u}$ & $a_{s}$ & $h_{s}$ & $a$ & $h_{d}$ \\
\hline
\end{tabular}

\begin{tabular}{llllllll}
\hline & $M_{\tilde{\chi}_{1}^{0}}$ & $M_{\tilde{\chi}_{2}^{0}}$ & $M_{\tilde{\chi}_{3}^{0}}$ & $M_{\tilde{\chi}_{4}^{0}}$ & $M_{\tilde{\chi}_{5}^{0}}$ & $M_{\tilde{\chi}_{1}^{+}}$ & $M_{\tilde{\chi}_{2}^{+}}$ \\
\hline OS1 & & & & & & & \\
Tree-level & 265.97 & 276.05 & 565.2 & 730.92 & 920.76 & 270.72 & 730.83 \\
One-loop & 273.22 & 282.48 & 565.2 & 730.78 & 914.39 & 278.02 & 730.83 \\
OS2 & & & & & & & \\
Tree-level & 265.97 & 276.05 & 565.2 & 730.78 & 920.76 & 270.72 & 730.69 \\
One-loop & 273.22 & 282.48 & 565.2 & 730.78 & 914.39 & 278.02 & 730.83 \\
DR & & & & & & & \\
Tree-level & 265.5 & 276.2 & 563.47 & 694.32 & 920.76 & 270.38 & 694.18 \\
One-loop & 273.21 & 282.44 & 565.21 & 730.01 & 914.39 & 278.01 & 729.86 \\
Main component & $\tilde{H}_{d}^{0}$ & $\tilde{H}_{u}^{0}$ & $\tilde{B}$ & $\tilde{W}_{3}$ & $\tilde{S}$ & $\tilde{H}^{+}$ & $\tilde{W}^{+}$ \\
\hline
\end{tabular}

The Higgs boson masses and their main composition in terms of singlet/doublet and scalar/pseudoscalar components at two-loop order $\mathcal{O}\left(\alpha_{t} \alpha_{s}+\alpha_{t}^{2}\right)$ for $\overline{\mathrm{DR}}$ renormalization in the top/stop sector computed by NMSSMCALCEW, are summarized in Table 2.

For scenariol, we present in Table 3 the tree-level and loop-corrected masses of the neutralinos and charginos in the three different renormalization schemes and for the Denner description. As expected the wino-like neutralino and the wino-like chargino which couple to the electroweak gauge bosons, get significant loop corrections in the $\overline{\mathrm{DR}}$ scheme. The one-loop corrected masses themselves, however, barely differ in the three renormalization schemes so that the remaining theoretical error due to missing higher-order corrections is very small.

We vary the phases of the gaugino mass parameters $M_{1}$ and $M_{2}, \varphi_{M_{1}}$ and $\varphi_{M_{2}}$, in order to study their effect on the loop-corrected neutralino and chargino masses. Note that these complex phases have negligible impact on the Higgs sector $[16,18]$. We use the $\overline{\mathrm{DR}}$ scheme for this analysis and show in the upper plots of Fig. 10 the loop corrections to the electroweakino masses,

$$
\begin{aligned}
\Delta M_{x} & =M_{x}^{\text {loop }}-m_{x}^{\text {tree }}, \\
x & =\tilde{\chi}_{i}^{0}, \tilde{\chi}_{j}^{ \pm} \quad(i=1, \ldots, 5, j=1,2),
\end{aligned}
$$

as function of $\varphi_{M_{1}}$ (left) and $\varphi_{M_{2}}$ (right). We apply a subtraction $\delta M_{x}$ to the mass corrections $\Delta M_{x}$ of the different electroweakinos that allows us to show all corrections, which can be very different in size, in one plot. In the lower plots of Fig. 10 we show the differences

$\operatorname{diff}_{x}=M_{x}^{\text {loop, } \mathrm{D}}-M_{x}^{\text {loop, EMT }}$, with $x$ denoting any electroweakino, between the loop corrected electroweakino masses computed using the Denner and the EMT descriptions presented in Sect. 3.1.2. As can be inferred from the upper plots, the wino-like neutralino $\tilde{\chi}_{4}^{0}$ (cyan line) and wino-like chargino $\tilde{\chi}_{2}^{+}$(black line) receive the largest loop corrections of about $35 \mathrm{GeV}$ in absolute values. The corrections to the Higgsino-like neutralinos $\tilde{\chi}_{1}^{0}$ (blue line) and $\tilde{\chi}_{2}^{0}$ (green line), the singlino-like neutralino $\tilde{\chi}_{0}^{5}$ (red line) and the Higgsino-like chargino $\tilde{\chi}_{1}^{+}$(pink line) range around $6-8 \mathrm{GeV}$. The correction to the bino-like neutralino $\tilde{\chi}_{3}^{0}$ (orange line) is somewhat smaller with values around 1.5-1.8 GeV. A small difference between the Denner and the EMT descriptions of about $1-2 \mathrm{MeV}$ is observed for the Higgsino-like neutralinos, $c f$. lower plots. We do not see any difference, however, for the chargino masses. This is because we have used the approximation in Eq. (3.100) for the loopcorrected chargino masses. There is a compensation between $\operatorname{Re} \Sigma_{i i}^{\tilde{\chi}^{+}, L s}\left(m_{\tilde{\chi}_{i}^{ \pm}}^{2}\right)$ and $\operatorname{Re} \Sigma_{i i}^{\tilde{\chi}^{+}, R s}\left(m_{\tilde{\chi}_{i}^{ \pm}}^{2}\right)$ that kills the effect of the imaginary part of the loop integral function.

In order to study the loop corrections on the decay widths, we computed the tree-level and loop-corrected decay widths, defined in Sect. 4.4, for the three different renormalization schemes OS1, OS2 and $\overline{\mathrm{DR}}$ for the scenariol. Note that we use the loop-corrected masses for external Higgs bosons, charginos and neutralinos not only in the loop-corrected decay widths but also in the tree-level ones. For illustration, we present in Table 4 for the decays of all Higgs bosons in all possible electroweakino final states the loop-corrected decay widths $\Gamma^{l} \equiv \Gamma_{\mathbf{Z}^{H}}^{\mathrm{SEW}}\left(H_{i} \rightarrow \tilde{\chi}_{j} \tilde{\chi}_{k}\right)$, the relative corrections $\delta\left(H_{i} \tilde{\chi}_{j} \tilde{\chi}_{k}\right)$ as defined in Eq. (5.10), and the loop-corrected branching ratios $\mathrm{BR}^{l} \equiv \mathrm{BR}_{\mathbf{Z}^{H}}^{\mathrm{SEW}}\left(H_{i} \rightarrow \tilde{\chi}_{j} \tilde{\chi}_{k}\right)$ using scenario 1 , for the OS1 and $\overline{\mathrm{DR}}$ renormalization schemes. We 


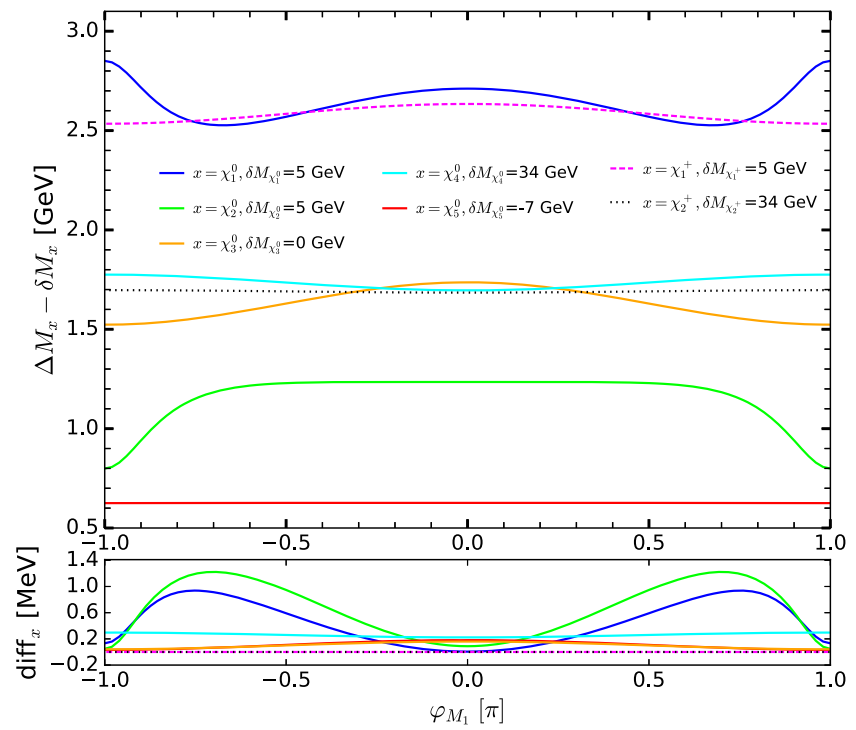

Fig. 10 Scenario1: Upper: Loop corrections to the electroweakino masses, $\Delta M_{x}$ (see text, for the definition), $x=\tilde{\chi}_{i}^{0}, \tilde{\chi}_{j}^{ \pm}(i=1, \ldots, 5$, $j=1,2$ ), as a function of $\varphi_{M_{1}}$ (left) and $\varphi_{M_{2}}$ (right) in the $\overline{\mathrm{DR}}$ scheme

found that $\Gamma^{l}$ is almost identical in the two OS schemes. The relative size of the loop corrections is below $10 \%$ in the OS scheme. The relative corrections in the $\overline{\mathrm{DR}}$ scheme are always larger than in the OS schemes. For some channels with small decay widths, we see significant corrections in the $\overline{\mathrm{DR}}$ scheme. For example in the decay channel $H_{4} \rightarrow \tilde{\chi}_{2}^{0} \tilde{\chi}_{2}^{0}$, the relative loop correction is $-0.13 \%$ in the OS scheme while it is $-20 \%$ in the $\overline{\mathrm{DR}}$ scheme. Based on our investigation, we conclude that it is better to use the OS scheme in the decays of the neutral Higgs bosons into electroweakinos. The largest uncertainty due to missing higher-order corrections that we estimate from the variation of the renormalization schemes is found to be $17 \%$ in the decay $H_{5} \rightarrow \tilde{\chi}_{1}^{0} \tilde{\chi}_{2}^{0}$. We also studied the difference between the Denner and EMT descriptions and did not observe any significant difference. Defining the difference as $\left(\Gamma_{x}^{\mathrm{D}}-\Gamma_{x}^{\mathrm{EMT}}\right) / \Gamma_{x}^{\mathrm{D}}$ with $\Gamma_{x}$ being the loop-corrected decay width for some Higgs decay into an electroweakino final state, we see that the differences are of per mille level for all investigated decays.

\subsection{Decays into a squark pair}

We start by discussing the top and bottom squark masses in the OS and $\overline{\mathrm{DR}}$ renormalization schemes defined in Sect. 3.1.3. We follow the SLHA convention where the input parameters $m_{\tilde{Q}_{3}}, m_{\tilde{t}_{R}}, m_{\tilde{b}_{R}}, A_{t}, A_{b}$ are $\overline{\mathrm{DR}}$ parameters at the scale $M_{\text {SUSY }}$. When we apply the OS scheme, these parameters must be translated into OS parameters by applying the

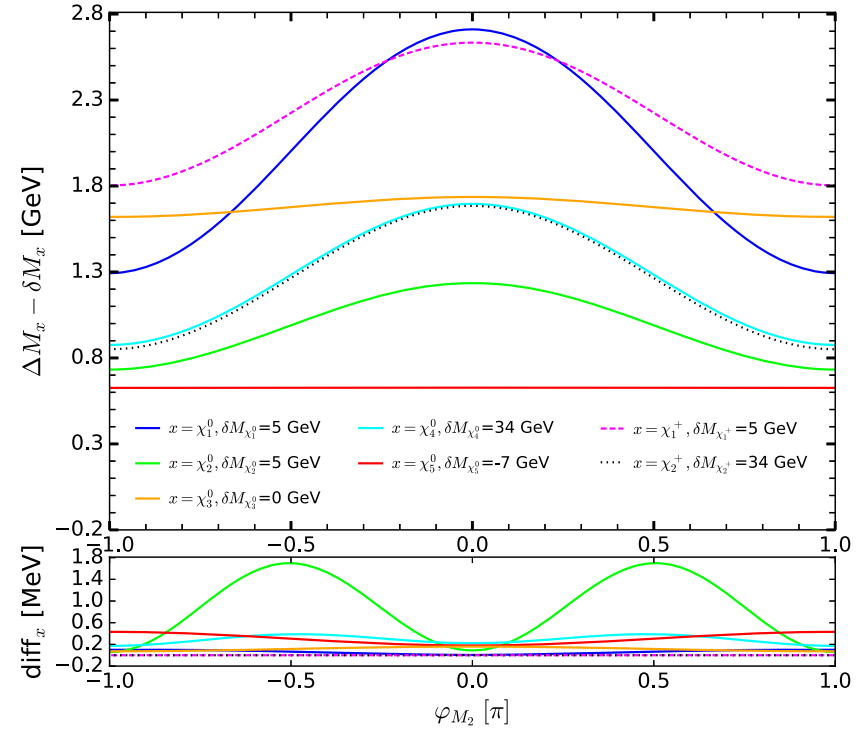

using the Denner description. The subtraction values $\delta M_{x}$ are speci-

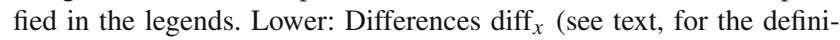
tion) between the loop-corrected masses using the Denner and the EMT descriptions

approximate transformation formula $(i=1,2)$

$X^{\mathrm{OSi}}=X^{\overline{\mathrm{DR}}}-\delta X^{\mathrm{finOSi}}$,

with $X=m_{\tilde{Q}_{3}}, m_{\tilde{t}_{R}}, m_{\tilde{b}_{R}}, A_{t}, A_{b}$ and the finite part of their OS counterterms denoted as $\delta X^{\text {finOSi }}$. We have used an iterative method to obtain a stable value of $\delta X^{\text {finOSi }}$. Note that we include both the NLO QCD and the full NLO EW contribution in the conversion Eq. (5.17). ${ }^{40}$ Using these OS parameters together with the OS top mass in the tree-level mass matrices, we obtain the top and bottom squark masses. When we apply the $\overline{\mathrm{DR}}$ renormalization scheme, the top pole mass has to be translated to the $\overline{\mathrm{DR}}$ top mass for which we follow the description in "Appendix C" of Ref. [18]. The $\overline{\mathrm{DR}}$ top and bottom masses together with the $\overline{\mathrm{DR}}$ parameters $m_{\tilde{Q}_{3}}, m_{\tilde{t}_{R}}, m_{\tilde{b}_{R}}, A_{t}, A_{b}$ are then used in the tree-level mass matrices to get the tree-level rotation matrices. They are subsequently used in the computation of the renormalized self-energies of the top and bottom squarks to obtain the loop-corrected squark masses as described in Sect. 3.4. In principle, we would expect that the loop-corrected masses computed in the $\overline{\mathrm{DR}}$ scheme are closer to the OS masses in the OS description if one includes more higher order corrections. We consider here a parameter point (scenario2) given by the following soft SUSY breaking masses and trilinear

\footnotetext{
40 This is a bit different from the Higgs mass calculation in NMSSMCALCEW where we include the NLO QCD correction and the NLO Yukawa correction of order $\mathcal{O}\left(\alpha_{t}\right)$ to the conversion in the OS renormalization scheme of the top/stop sector.
} 
Table 4 Scenario 1: Loop-corrected decay widths $\Gamma^{l}$, relative loop corrections $\delta$ and loop-corrected branching ratios $\mathrm{BR}^{l}$ of all kinematically allowed decays into chargino and neutralino pairs of heavy Higgs bosons in the OS1 and $\overline{\mathrm{DR}}$ renormalization schemes. The results in the OS2 scheme are nearly the same as in OS1 scheme

\begin{tabular}{|c|c|c|c|c|c|c|c|c|}
\hline & & $\Gamma^{l}(\mathrm{MeV})$ & $\delta(\%)$ & $\mathrm{BR}^{l}(\%)$ & & $\Gamma^{l}(\mathrm{MeV})$ & $\delta(\%)$ & $\mathrm{BR}^{l}(\%)$ \\
\hline \multirow[t]{2}{*}{$H_{2} \rightarrow \tilde{\chi}_{1}^{0} \tilde{\chi}_{1}^{0}$} & OS1 & 405.67 & 0.08 & 25.14 & $H_{2} \rightarrow \tilde{\chi}_{1}^{0} \tilde{\chi}_{2}^{0}$ & 0.43 & -0.08 & 0.03 \\
\hline & $\overline{\mathrm{DR}}$ & 407.74 & 8.4 & 25.15 & & 0.4 & -16.34 & 0.02 \\
\hline \multirow[t]{2}{*}{$H_{2} \rightarrow \tilde{\chi}_{2}^{0} \tilde{\chi}_{2}^{0}$} & OS1 & 404.44 & 0.07 & 25.06 & $H_{2} \rightarrow \tilde{\chi}_{1}^{+} \tilde{\chi}_{1}^{-}$ & 802.3 & 0.08 & 49.72 \\
\hline & $\overline{\mathrm{DR}}$ & 404.84 & 7.23 & 24.98 & & 807.18 & 9.03 & 49.8 \\
\hline \multirow{2}{*}{$H_{3} \rightarrow \tilde{\chi}_{1}^{0} \tilde{\chi}_{1}^{0}$} & OS1 & 321.53 & 0.08 & 22.01 & $H_{3} \rightarrow \tilde{\chi}_{1}^{0} \tilde{\chi}_{2}^{0}$ & 0.78 & -0.11 & 0.05 \\
\hline & $\overline{\mathrm{DR}}$ & 322.45 & 8.13 & 22.04 & & 0.73 & -16.24 & 0.05 \\
\hline \multirow[t]{2}{*}{$H_{3} \rightarrow \tilde{\chi}_{2}^{0} \tilde{\chi}_{2}^{0}$} & OS1 & 308.94 & 0.07 & 21.15 & $H_{3} \rightarrow \tilde{\chi}_{1}^{+} \tilde{\chi}_{1}^{-}$ & 633.52 & 0.08 & 43.38 \\
\hline & $\overline{\mathrm{DR}}$ & 309.0 & 7.08 & 21.12 & & 635.37 & 8.79 & 43.42 \\
\hline \multirow{2}{*}{$H_{4} \rightarrow \tilde{\chi}_{1}^{0} \tilde{\chi}_{1}^{0}$} & OS1 & 102.86 & 0.02 & 0.8 & $H_{5} \rightarrow \tilde{\chi}_{1}^{0} \tilde{\chi}_{1}^{0}$ & 151.31 & -0.01 & 1.18 \\
\hline & $\overline{\mathrm{DR}}$ & 89.4 & -11.31 & 0.69 & & 132.77 & -13.56 & 1.04 \\
\hline \multirow[t]{2}{*}{$H_{4} \rightarrow \tilde{\chi}_{1}^{0} \tilde{\chi}_{2}^{0}$} & OS1 & 17.87 & 0.08 & 0.14 & $H_{5} \rightarrow \tilde{\chi}_{1}^{0} \tilde{\chi}_{2}^{0}$ & 9.29 & 0.24 & 0.07 \\
\hline & $\overline{\mathrm{DR}}$ & 14.59 & -11.68 & 0.11 & & 7.65 & 1.94 & 0.06 \\
\hline \multirow{2}{*}{$H_{4} \rightarrow \tilde{\chi}_{1}^{0} \tilde{\chi}_{3}^{0}$} & OS1 & 395.9 & -5.5 & 4.59 & $H_{5} \rightarrow \tilde{\chi}_{1}^{0} \tilde{\chi}_{3}^{0}$ & 423.04 & 0.02 & 3.3 \\
\hline & $\overline{\mathrm{DR}}$ & 451.86 & 6.59 & 3.5 & & 448.9 & 7.83 & 3.5 \\
\hline \multirow{2}{*}{$H_{4} \rightarrow \tilde{\chi}_{1}^{0} \tilde{\chi}_{4}^{0}$} & OS1 & 1448.21 & -0.03 & 11.22 & $H_{5} \rightarrow \tilde{\chi}_{1}^{0} \tilde{\chi}_{4}^{0}$ & 1161.18 & -0.02 & 9.05 \\
\hline & $\overline{\mathrm{DR}}$ & 1438.6 & -3.68 & 11.15 & & 1157.98 & -2.81 & 9.03 \\
\hline \multirow[t]{2}{*}{$H_{4} \rightarrow \tilde{\chi}_{1}^{0} \tilde{\chi}_{5}^{0}$} & OS1 & 194.66 & -0.02 & 1.51 & $H_{5} \rightarrow \tilde{\chi}_{1}^{0} \tilde{\chi}_{5}^{0}$ & 338.35 & -0.02 & 2.64 \\
\hline & $\overline{\mathrm{DR}}$ & 195.02 & -1.63 & 1.51 & & 341.12 & -1.58 & 2.66 \\
\hline \multirow{2}{*}{$H_{4} \rightarrow \tilde{\chi}_{2}^{0} \tilde{\chi}_{2}^{0}$} & OS1 & 27.86 & -0.13 & 0.22 & $H_{5} \rightarrow \tilde{\chi}_{2}^{0} \tilde{\chi}_{2}^{0}$ & 34.6 & -0.1 & 0.27 \\
\hline & $\overline{\mathrm{DR}}$ & 25.54 & -20.08 & 0.2 & & 32.72 & -15.35 & 0.26 \\
\hline \multirow[t]{2}{*}{$H_{4} \rightarrow \tilde{\chi}_{2}^{0} \tilde{\chi}_{3}^{0}$} & OS1 & 425.3 & 0.02 & 3.29 & $H_{5} \rightarrow \tilde{\chi}_{2}^{0} \tilde{\chi}_{3}^{0}$ & 413.74 & 0.01 & 3.22 \\
\hline & $\overline{\mathrm{DR}}$ & 450.13 & 7.77 & 3.49 & & 438.11 & 6.48 & 3.42 \\
\hline \multirow{2}{*}{$H_{4} \rightarrow \tilde{\chi}_{2}^{0} \tilde{\chi}_{4}^{0}$} & OS1 & 1125.46 & -0.02 & 8.72 & $H_{5} \rightarrow \tilde{\chi}_{2}^{0} \tilde{\chi}_{4}^{0}$ & 1379.31 & -0.03 & 10.75 \\
\hline & $\overline{\mathrm{DR}}$ & 1116.5 & -3.34 & 8.66 & & 1366.13 & -3.91 & 10.66 \\
\hline \multirow[t]{2}{*}{$H_{4} \rightarrow \tilde{\chi}_{2}^{0} \tilde{\chi}_{5}^{0}$} & OS1 & 382.4 & -0.02 & 2.96 & $H_{5} \rightarrow \tilde{\chi}_{2}^{0} \tilde{\chi}_{5}^{0}$ & 180.21 & -0.02 & 1.4 \\
\hline & $\overline{\mathrm{DR}}$ & 381.89 & -2.35 & 2.96 & & 180.34 & -1.86 & 1.41 \\
\hline \multirow{2}{*}{$H_{4} \rightarrow \tilde{\chi}_{3}^{0} \tilde{\chi}_{3}^{0}$} & OS1 & 1.2 & 0.19 & 0.01 & $H_{5} \rightarrow \tilde{\chi}_{3}^{0} \tilde{\chi}_{3}^{0}$ & 1.19 & 0.18 & 0.01 \\
\hline & $\overline{\mathrm{DR}}$ & 1.29 & 28.87 & 0.01 & & 1.29 & 27.81 & 0.01 \\
\hline \multirow[t]{2}{*}{$H_{4} \rightarrow \tilde{\chi}_{3}^{0} \tilde{\chi}_{4}^{0}$} & OS1 & 3.18 & 0.05 & 0.02 & $H_{5} \rightarrow \tilde{\chi}_{3}^{0} \tilde{\chi}_{4}^{0}$ & 1.48 & 0.06 & 0.01 \\
\hline & $\overline{\mathrm{DR}}$ & 2.95 & -2.79 & 0.02 & & 1.43 & 0.89 & 0.01 \\
\hline \multirow[t]{2}{*}{$H_{4} \rightarrow \tilde{\chi}_{1}^{+} \tilde{\chi}_{1}^{-}$} & OS1 & 393.59 & -0.01 & 3.05 & $H_{5} \rightarrow \tilde{\chi}_{1}^{+} \tilde{\chi}_{1}^{-}$ & 257.68 & -0.03 & 2.01 \\
\hline & $\overline{\mathrm{DR}}$ & 343.95 & -13.61 & 2.67 & & 215.16 & -19.09 & 1.68 \\
\hline \multirow[t]{2}{*}{$H_{4} \rightarrow \tilde{\chi}_{1}^{+} \tilde{\chi}_{2}^{-}$} & OS1 & 2440.51 & -0.01 & 18.91 & $H_{5} \rightarrow \tilde{\chi}_{1}^{+} \tilde{\chi}_{2}^{-}$ & 2482.01 & -0.01 & 19.34 \\
\hline & $\overline{\mathrm{DR}}$ & 2461.41 & -0.61 & 19.0 & & 2498.8 & -0.9 & 19.49 \\
\hline \multirow[t]{2}{*}{$H_{4} \rightarrow \tilde{\chi}_{2}^{+} \tilde{\chi}_{1}^{-}$} & OS1 & 2440.51 & -0.01 & 18.91 & $H_{5} \rightarrow \tilde{\chi}_{2}^{+} \tilde{\chi}_{1}^{-}$ & 2482.01 & -0.01 & 19.34 \\
\hline & $\overline{\mathrm{DR}}$ & 2461.41 & -0.61 & 19.0 & & 2498.8 & -0.9 & 19.49 \\
\hline
\end{tabular}

couplings 41

$$
\begin{aligned}
m_{\tilde{u}_{R}, \tilde{c}_{R}} & =m_{\tilde{d}_{R}, \tilde{s}_{R}}=m_{\tilde{Q}_{1,2}}=m_{\tilde{L}_{1,2}}=m_{\tilde{e}_{R}, \tilde{\mu}_{R}}=3 \mathrm{TeV}, \\
m_{\tilde{t}_{R}} & =623 \mathrm{GeV}
\end{aligned}
$$

$\overline{41}$ This parameter point is allowed by HiggsBounds5.3.2 and its $\chi^{2}$ computed by HiggsSignals2.2.3 is consistent with an SM $\chi^{2}$ less than $1 \sigma$.

$$
\begin{aligned}
m_{\tilde{Q}_{3}} & =1180 \mathrm{GeV}, m_{\tilde{b}_{R}}=m_{\tilde{L}_{3}}=m_{\tilde{\tau}_{R}}=33 \mathrm{TeV} \\
\left|A_{u, c, t}\right| & =1760 \mathrm{GeV},\left|A_{d, s, b}\right|=2000 \mathrm{GeV} \\
\left|A_{e, \mu, \tau}\right| & =2000 \mathrm{GeV} \\
\left|M_{1}\right| & =1000 \mathrm{GeV},\left|M_{2}\right|=1251 \mathrm{GeV} \\
\left|M_{3}\right| & =2364 \mathrm{GeV}
\end{aligned}
$$


Table 5 Scenario2: The tree-level and one-loop corrected stop and sbottom masses in the $\overline{\mathrm{DR}}$ and OS schemes

\begin{tabular}{lllll}
\hline & $m_{\tilde{t}_{1}}(\mathrm{GeV})$ & $m_{\tilde{t}_{2}}(\mathrm{GeV})$ & $m_{\tilde{b}_{1}}(\mathrm{GeV})$ & $m_{\tilde{b}_{2}}(\mathrm{GeV})$ \\
\hline OS & & & & \\
Tree & 334 & 1166 & 1122 & 2297 \\
$\quad 1$ loop & 334 & 1166 & 1125 & 2297 \\
$\overline{\text { DR }}$ & & & & \\
Tree & 585 & 1216 & 1181 & 3000 \\
1 loop & 341 & 1152 & 1152 & 2294 \\
\hline
\end{tabular}

with the $\mathrm{CP}$ phases given by

$\varphi_{A_{u, c, t}}=\varphi_{A_{d, s, b}}=\varphi_{A_{e, \mu, \tau}}=\varphi_{M_{1}}=\varphi_{M_{2}}=\varphi_{M_{3}}=0$.

The remaining input parameters have been set to

$$
\begin{aligned}
\lambda & =0.106, \quad \kappa=-0.238, \quad \operatorname{Re}\left(A_{\kappa}\right)=-647 \mathrm{GeV}, \\
\mu_{\mathrm{eff}} & =-603 \mathrm{GeV}, \\
\varphi_{u} & =0, \quad \tan \beta=17.5, \quad M_{H^{ \pm}}=1867 \mathrm{GeV} .
\end{aligned}
$$

With the given $\overline{\mathrm{DR}}$ parameters of the squark sector, we obtain their corresponding OS parameters

$$
\begin{aligned}
m_{\tilde{Q}_{3}} & =1120 \mathrm{GeV}, \quad m_{\tilde{t}_{R}}=402 \mathrm{GeV}, \quad m_{\tilde{b}_{R}}=2997 \mathrm{GeV}, \\
A_{t} & =1720 \mathrm{GeV}, \quad A_{b}=-581 \mathrm{GeV} .
\end{aligned}
$$

The tree-level and loop-corrected masses of the stops and sbottoms in the OS and $\overline{\mathrm{DR}}$ scheme are shown in Table 5. We see that for the $\overline{\mathrm{DR}}$ scheme there are large changes between the tree-level and one-loop masses, in particular for the lightest stop $\tilde{t}_{1}$. The loop-corrected masses, however, are then closer to each other in both schemes, as expected. The maximum difference is found for the light sbottom $\tilde{b}_{1}$ mass, where the $\mathrm{OS}$ and $\overline{\mathrm{DR}}$ results differ by $27 \mathrm{GeV}$ at one-loop order (compared to $59 \mathrm{GeV}$ at tree level). The two-loop corrected neutral Higgs boson masses at $\mathcal{O}\left(\alpha_{t} \alpha_{s}+\alpha_{t}^{2}\right)$ together with their respective main component are displayed in Table 6 . The SM-like Higgs boson mass is around $124 \mathrm{GeV}$ while the remaining Higgs spectrum is quite heavy with masses above $1.6 \mathrm{TeV}$.

We now turn on the complex phase of $A_{t}$. In the left plot of Fig. 11, we show the tree-level (black), NLO EW (green), NLO QCD (blue), and full, i.e. NLO QCD+EW, (red) correc- tions to the partial width of the decay $H_{4} \rightarrow \tilde{t}_{1} \tilde{t}_{1}^{*}$ as function of the phase $\varphi_{A_{t}}$ in both the OS (full lines) and the $\overline{\mathrm{DR}}$ (dashed lines) schemes while their corresponding branching ratios are depicted in the right plot. The decay $H_{4} \rightarrow \tilde{t}_{1} \tilde{t}_{1}^{*}$ vanishes in the CP-conserving limit where $H_{4}$, which is $a$-like in scenario2, is a CP-odd Higgs boson. (Note that CP-odd Higgs bosons at tree-level only couple to two different stops.) In the OS scheme, the relative EW corrections $\delta$ (see Eq. (5.10) for the definition) vary in the range $(-6 \%,-10 \%)$ and the QCD corrections in the range $(-4 \%,-8 \%)$ depending on the phase $\varphi_{A_{t}}$ that is varied from zero to $\pm \pi / 2$. In the $\overline{\mathrm{DR}}$ scheme, the relative EW corrections are of order $-10 \%$ and the relative QCD corrections of $22 \%$ and depend slightly on the phase $\varphi_{A_{t}}$. We define the relative differences between the OS and $\overline{\mathrm{DR}}$ decay widths and branching ratios, as

$\Delta_{\Gamma}=\left|\frac{\Gamma_{\mathbf{Z}^{H}}^{\mathrm{OS}}-\Gamma_{\mathbf{Z}^{H}}^{\overline{\mathrm{DR}}}}{\Gamma_{\mathbf{Z}^{H}}^{\mathrm{OS}}}\right|$,

and

$\Delta_{\mathrm{BR}}=\left|\frac{\mathrm{BR}_{\mathbf{Z}^{H}}^{\mathrm{OS}}-\mathrm{BR}_{\mathbf{Z}^{H}}^{\overline{\mathrm{DR}}}}{\mathrm{BR}_{\mathbf{Z}^{H}}^{\mathrm{OS}}}\right|$,

respectively. For $\varphi_{A_{t}}=-\pi / 2$, the relative difference $\Delta_{\Gamma}$ of the partial decay widths between the OS and $\overline{\mathrm{DR}}$ schemes is then about $40 \%$ at tree-level while it reduces to $4 \%$ when both QCD and EW corrections are included, so that at one-loop level we clearly see a reduction of the theoretical error due to missing higher-order corrections. For the relative error in the branching ratios we find values between $32 \%$ and $27 \%$ at tree level and between $0.3 \%$ and $3 \%$ at one-loop order including both the EW and QCD corrections while the phase $\varphi_{A_{t}}$ is varied from $\pm \pi / 2$ to zero.

For the decay $H_{4}\left(\equiv H_{a}\right) \rightarrow \tilde{t}_{1} \tilde{t}_{2}^{*}$, we show in the upper panels of Fig. 12 the partial decay width (left) and branching ratio (right) at tree-level (black), NLO EW (green), NLO QCD (blue), and NLO EW+QCD (red) as a function of $\varphi_{A_{t}}$, both for the OS (full) and $\overline{\mathrm{DR}}$ (dashed) scheme. In the middle panels we show the relative NLO EW, NLO QCD and NLO $\mathrm{EW}+\mathrm{QCD}$ corrections which are defined as

$\delta_{\Gamma}=\frac{\Gamma_{\mathbf{Z}^{H}}^{\mathrm{EW} / \mathrm{QCD} / \mathrm{EW}+\mathrm{QCD}}-\Gamma_{\mathbf{Z}^{H}}^{\text {tree }}}{\Gamma_{\mathbf{Z}^{H}}^{\text {Tree }}}$

Table 6 Scenario2: Mass values in $\mathrm{GeV}$ and main components of the neutral Higgs bosons at two-loop order $\mathcal{O}\left(\alpha_{t} \alpha_{s}+\alpha_{t}^{2}\right)$ obtained by using the $\overline{\mathrm{DR}}$ renormalization in the top/stop sector

\begin{tabular}{llllll}
\hline & $H_{1}$ & $H_{2}$ & $H_{3}$ & $H_{4}$ & $H_{5}$ \\
\hline Two-loop $\mathcal{O}\left(\alpha_{t} \alpha_{s}+\alpha_{t}^{2}\right)$ & 123.63 & 1621.65 & 1865.39 & 1895.83 & 2538.29 \\
Main component & $h_{u}$ & $a_{s}$ & $h_{d}$ & $a$ & $h_{s}$ \\
\hline
\end{tabular}



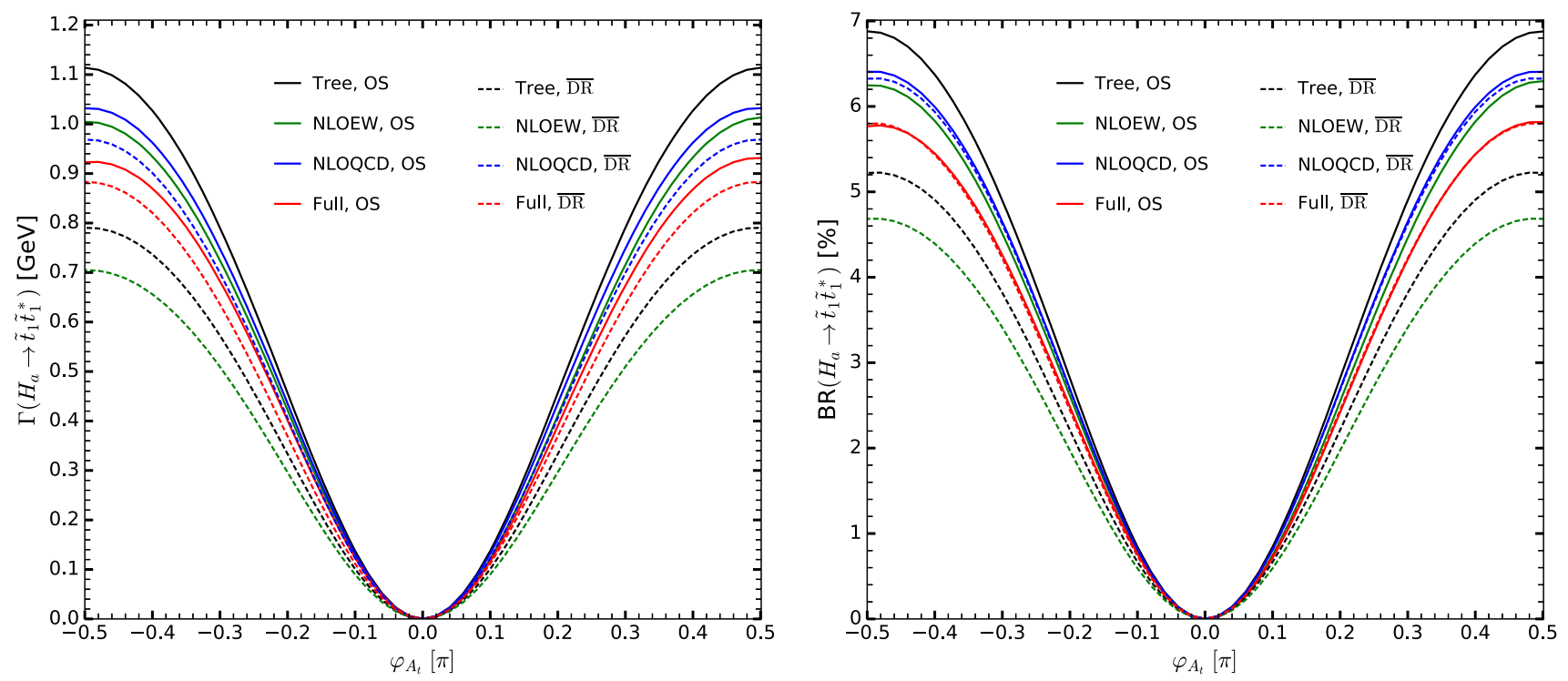

Fig. 11 Scenario2: Tree-level (black), NLO EW (green), NLO QCD (blue) and full (red) partial width (left) and branching ratio (right) of the decay $H_{4} \rightarrow \tilde{t}_{1} \tilde{t}_{1}^{*}\left(H_{4}\right.$ is $a$-like) as function of the complex phase $\varphi_{A_{t}}$. They are shown for the OS (full) and $\overline{\mathrm{DR}}$ scheme (dashed)

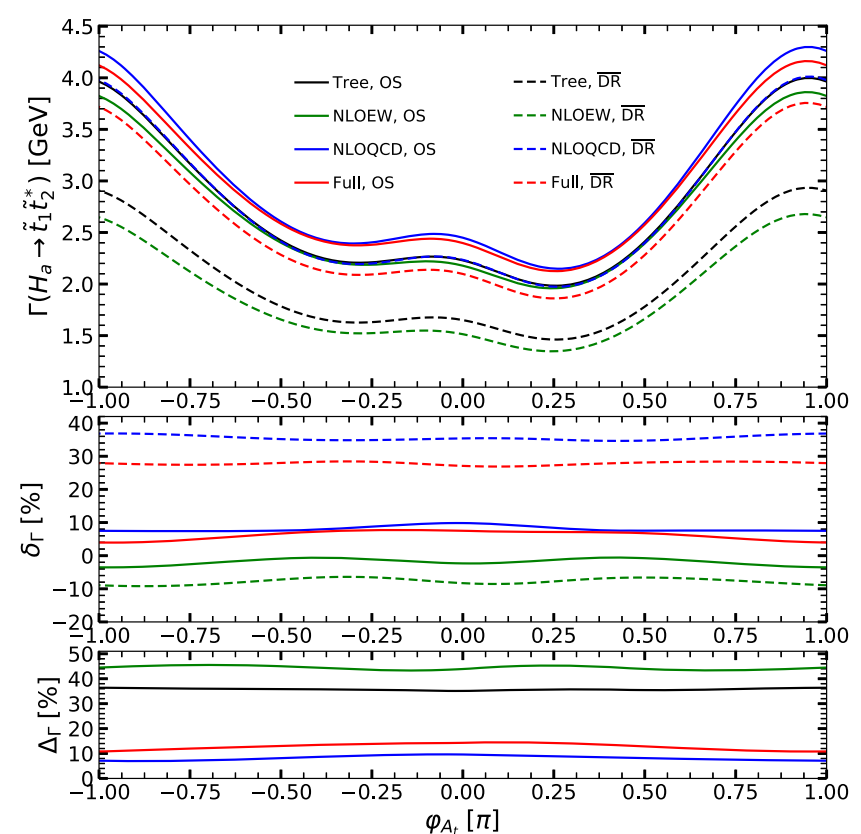

Fig. 12 Scenario2: Upper: Tree-level (black), NLO EW (green), NLO QCD (blue) and full (red) partial width (left) and branching ratio (right) of the decay $H_{4}\left(=H_{a}\right) \rightarrow \tilde{t}_{1} \tilde{t}_{2}^{*}$ as function of the complex phase $\varphi_{A_{t}}$. They are shown for the OS (full) and $\overline{\mathrm{DR}}$ (dashed) scheme. Middle: Relative EW, QCD and EW+QCD correc-

and

$$
\delta_{\mathrm{BR}}=\frac{\mathrm{BR}_{\mathbf{Z}^{H}}^{\mathrm{EW} / \mathrm{QCD} / \mathrm{EW}+\mathrm{QCD}}-\mathrm{BR}_{\mathbf{Z}^{H}}^{\text {tree }}}{\mathrm{BR}_{\mathbf{Z}^{H}}^{\text {Tree }}},
$$

respectively. The lower panels display the relative differences between the OS and $\overline{\mathrm{DR}}$ decay widths and branching ratios,

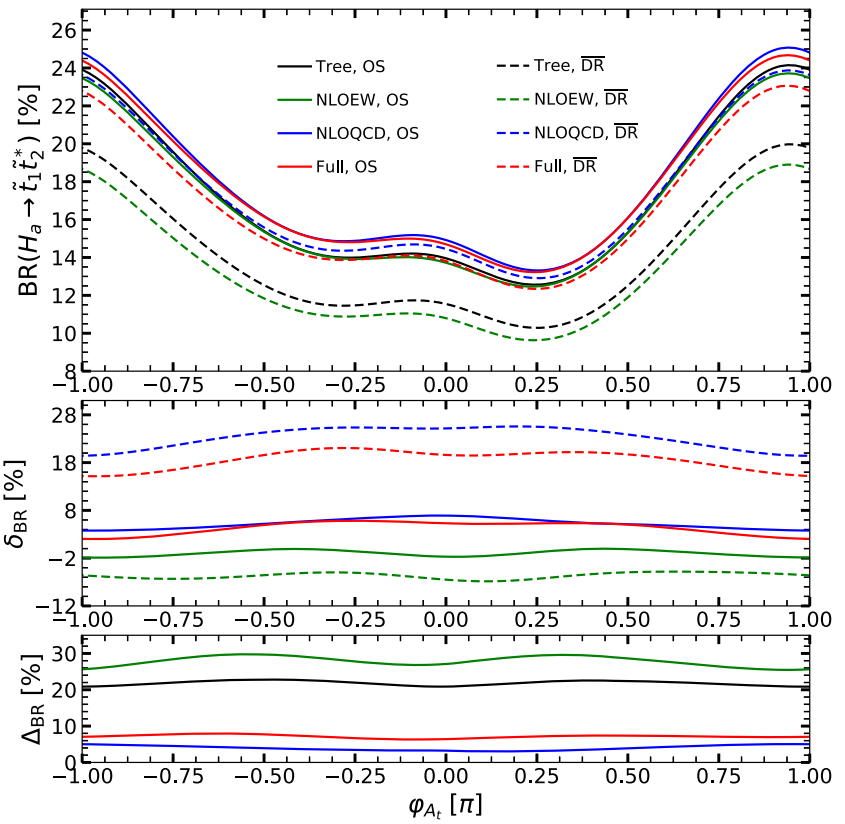

tions $\delta$ (see text, for definition) for the decay width (left) and branching ratio (right) in the OS (full) and $\overline{\mathrm{DR}}$ (dashed) scheme. Lower: Relative differences $\Delta$ between the OS and $\overline{\mathrm{DR}}$ scheme (see text, for definition) for the decay width (left) and branching ratio (right)

$\Delta_{\Gamma}$ and $\Delta_{\mathrm{BR}}$, as defined in Eqs. (5.22) and (5.23), respectively. The corrections vary slightly with the phase $\varphi_{A_{t}}$. The EW corrections are negative in both schemes while the QCD corrections are positive and of the same order of magnitude. This shows the importance to include both types of corrections to make reliable predictions. Overall, the relative cor- 
rections $\delta$ in the $\overline{\mathrm{DR}}$ scheme are larger than in the OS scheme. As can be inferred from the bottom left panel of Fig. 12, for $\varphi_{A_{t}}=0$, the relative difference in the partial width, $\Delta_{\Gamma}$, between the OS and $\overline{\mathrm{DR}}$ scheme at tree level is about $37 \%$ and decreases dramatically to less than $12 \%$ when both the EW and the QCD corrections are included. ${ }^{42}$ Similar results are found for the branching ratios, presented on the right plots of Fig. 12, with smaller values of $21 \%$ at tree level and $7 \%$ at full one-loop order. Note that in the right hand side plots we treated the decays $H_{a} \rightarrow \tilde{t}_{1} \tilde{t}_{2}^{*}$ and $H_{a} \rightarrow \tilde{t}_{2} \tilde{t}_{1}^{*}$ at the same level of precision while all other decays are computed at the highest possible precision.

In the CP-invariant scenario the decay width of decay $H_{a} \rightarrow \tilde{t}_{1} \tilde{t}_{2}^{*}$ is equal to the one of its charge conjugate decay $H_{a} \rightarrow \tilde{t}_{2} \tilde{t}_{1}^{*}$. For non-vanishing $\varphi_{A_{t}}$, however, the CP asymmetry, defined as

$\delta_{\mathrm{CP}}=\frac{\Gamma\left(H_{a} \rightarrow \tilde{t}_{1} \tilde{t}_{2}^{*}\right)-\Gamma\left(H_{a} \rightarrow \tilde{t}_{2} \tilde{t}_{1}^{*}\right)}{\Gamma\left(H_{a} \rightarrow \tilde{t}_{1} \tilde{t}_{2}^{*}\right)+\Gamma\left(H_{a} \rightarrow \tilde{t}_{2} \tilde{t}_{1}^{*}\right)}$,

is non-zero. In Fig. 13 we show the $\mathrm{CP}$ asymmetry as a function of $\varphi_{A_{t}}$. We see that the $\mathrm{CP}$ asymmetry appears already at tree-level, which results from the imaginary part of the WFR factor $\mathbf{Z}^{H}$ and the imaginary part of the tree-level couplings $g_{h_{i}} \tilde{q}_{j} \tilde{q}_{k}^{*}$. The relative change of the asymmetry due to loop corrections ranges between $18 \%$ and $-9 \%$ in the OS scheme while in the $\overline{\mathrm{DR}}$ scheme it is about $8 \%$ when the phase $\varphi_{A_{t}}$ is varied from $-\pi / 4$ to $\pi / 4$.

We present in Fig. 14 the same plots for the decay widths and branching ratios as in Fig. 12 but for the decay of $H_{3} \rightarrow \tilde{t}_{1} \tilde{t}_{2}^{*}$ which is the dominant decay channel of $H_{3}$. In scenario $2 \mathrm{H}_{3}$ is $h_{d}$-like. For both the OS and the $\overline{\mathrm{DR}}$ scheme the NLO EW corrections to the decay width are negative and the relative corrections $\delta_{\Gamma}$ are around $-3 \%$ in the OS and $-8 \%$ in the $\overline{\mathrm{DR}}$ scheme. The NLO QCD corrections on the other hand are positive and their relative size can reach $8 \%$ in the OS scheme and around $37 \%$ in the $\overline{\mathrm{DR}}$ scheme. For $\varphi_{A_{t}}=0$, the difference between the decay widths in the OS and the $\overline{\mathrm{DR}}$ scheme, $\Delta_{\Gamma}$, is about $36 \%$ at tree-level and reduces to $12 \%$ at full one-loop level. The corresponding numbers for the branching ratios are similar.

We have observed that for this parameter point the EW and QCD corrections in the OS scheme are smaller than in the $\overline{\mathrm{DR}}$ scheme. In the OS scheme we have seen that this is due to a cancellation between the genuine triangle diagram contribution and the counterterm contribution while this does not happen in the $\overline{\mathrm{DR}}$ scheme. Overall the inclusion of the

\footnotetext{
42 When we only include the EW corrections the scheme dependence increases when going from tree- to one-loop level. Overall, the behavior is as expected, however, when the full set of corrections is included. This shows that care has to be taken, when estimating the theoretical uncertainty due to missing higher-order corrections based on a change of the renormalization schemes, if not all corrections of a given loop order are included. See also Ref. [18] for a similar discussion.
}

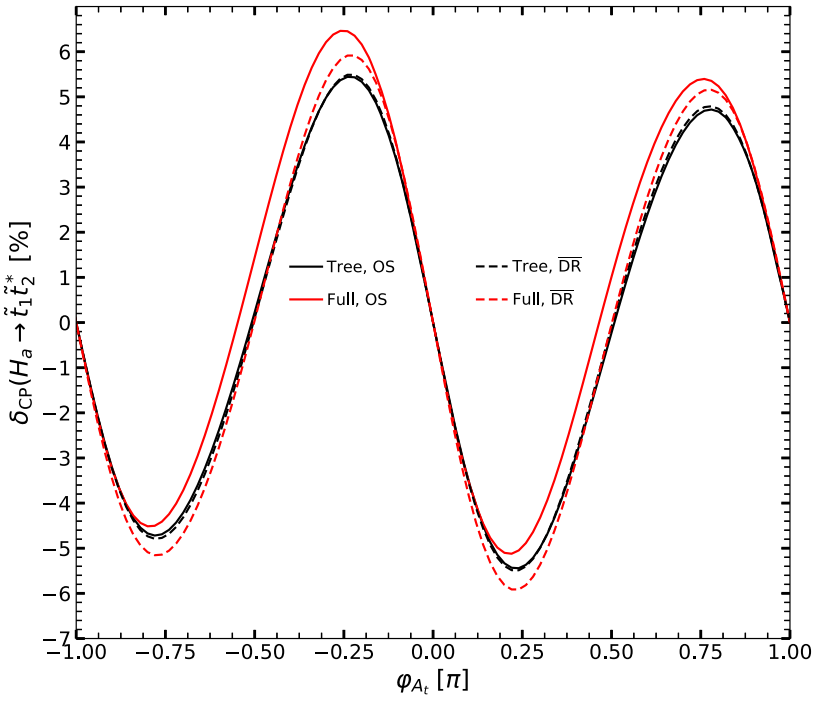

Fig. $13 \mathrm{CP}$-asymmetry $\delta_{\mathrm{CP}}$ as function of the complex phase $\varphi_{A_{t}}$ at tree level (black) and including both the $\mathrm{EW}$ and the QCD corrections (red) in the $\overline{\mathrm{DR}}$ (dashed) and OS (full) scheme

QCD corrections in addition to the EW corrections reduces the difference between the $\mathrm{OS}$ and $\overline{\mathrm{DR}}$ results.

\subsection{Gauge-parameter dependence in the decay of the neutral Higgs into fermions}

Inspired by the investigation of the gauge-parameter dependence in the computation of the loop-corrected Higgs masses and the decay width of the decay $H^{ \pm} \rightarrow W^{ \pm} H_{i}$ in Ref. [74], we investigate in this section the gauge-parameter dependence in the decays $h_{i} \rightarrow f \bar{f} .{ }^{43}$ For the other decays computed in this study, we do not present a detailed study. In fact, these other decay channels are only relevant for heavy Higgs bosons, for which the gauge-parameter dependence is negligible because of the smallness of the loop-corrections to their masses. However, as pointed out in Ref. [74], the gauge-parameter dependence can be significant for light neutral Higgs bosons receiving large loop-corrections to their masses. Following the strategy presented in Ref. [74], we compute the loop-corrected Higgs masses, the WFR factor $\mathbf{Z}^{H}, \delta \mathcal{M}^{\mathrm{rem}, S / P}$, and $\delta \mathcal{M}_{G Z \text {,mix }}$ in Eqs. (4.13) and (4.14) in a general $R_{\xi}$ gauge.

Only the NLO EW contributions, which are affected by our treatment of the gauge parameters, are recalculated in this section; the other corrections will be kept unchanged as in Eq. (4.12). We now expand the WFR factor $\mathbf{Z}^{H}$ to retain only

\footnotetext{
${ }^{43}$ It should be noted that a detailed study of the gauge dependence in the computation of the loop-corrected Higgs masses and the $h_{i} \rightarrow f \bar{f}$ decay widths has been presented recently in Ref. [77].
} 

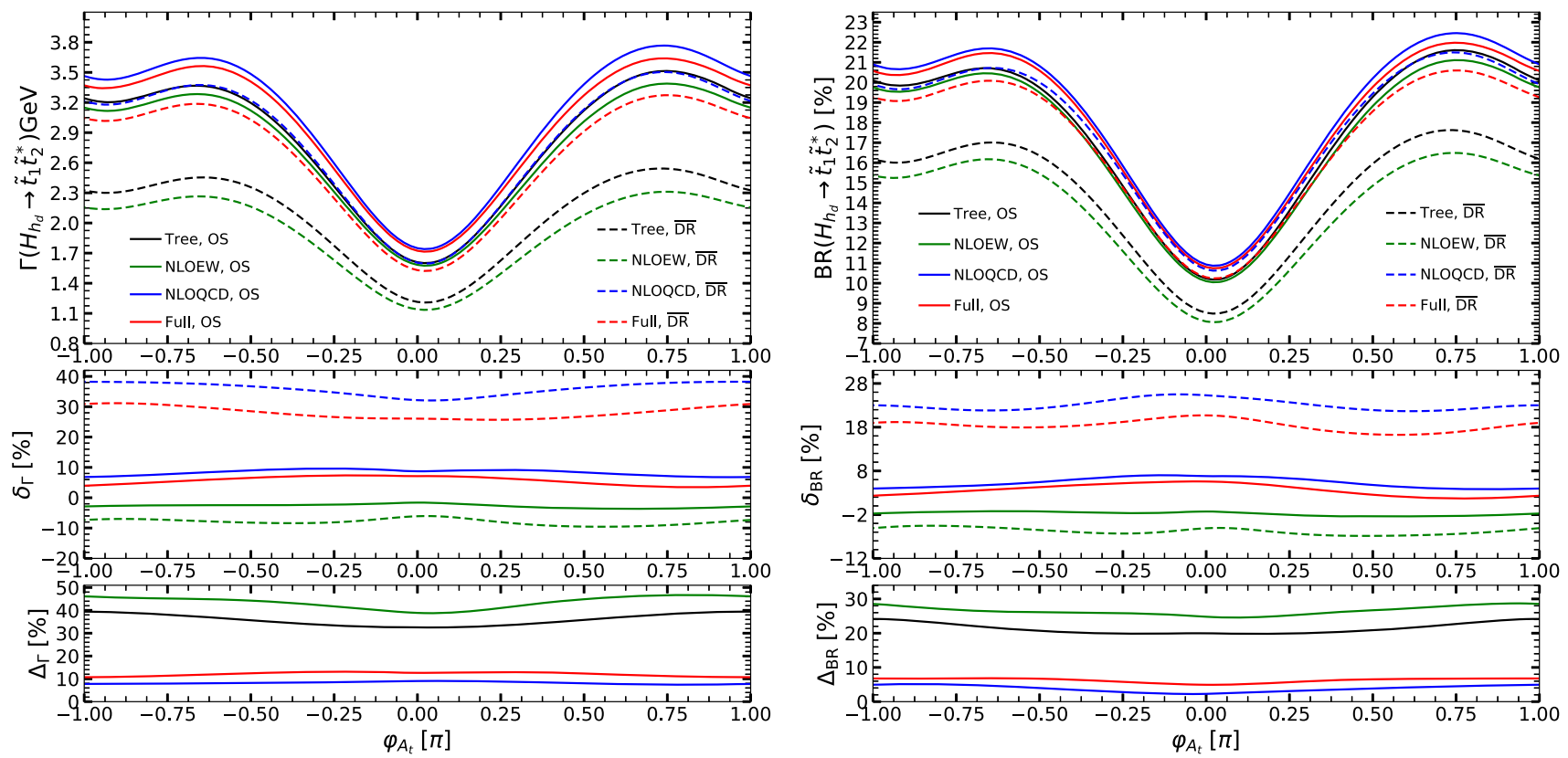

Fig. 14 Similar to Fig. 12 but for the decay $H_{h_{d}} \rightarrow \tilde{t}_{1} \tilde{t}_{2}^{*}$

the pure one-loop terms, so that we define the pure one-loop correction to $\Gamma_{H_{i} \rightarrow q \bar{q}}^{S / P}$ as

$$
\begin{aligned}
\Gamma_{H_{i} \rightarrow q \bar{q}}^{S, 1 l}= & \left(\tilde{g}_{h_{i} q \bar{q}}^{S}\right)\left(\tilde{s}_{h_{i} q \bar{q}}^{S}\right)^{*} \\
& +2 \operatorname{Re}\left[\tilde{g}_{h_{i} q \bar{q}}^{S}\left(\delta \mathcal{M}^{\mathrm{rem}, S}\left(h_{i} \rightarrow q \bar{q}\right)+\sum_{k=1}^{5} \delta \mathbf{Z}_{i k}^{1 l} \tilde{s}_{h_{k} q \bar{q}}^{S}\right)^{*}\right] \\
& +2 \operatorname{Re}\left[\left(\tilde{g}_{h_{i} q \bar{q}}^{S}\right)\left(\delta_{\text {sub }}^{S}\left(h_{i} \rightarrow q \bar{q}\right)\right)^{*}\right]
\end{aligned}
$$

$$
\begin{aligned}
& \left.\left.+\sum_{k=1}^{5} \delta \mathbf{Z}_{i k}^{1 l} \tilde{g}_{h_{k} q \bar{q}}^{P}+\delta \mathcal{M}_{G Z, \text { mix }}\left(h_{i} \rightarrow q \bar{q}\right)\right)^{*}\right] \\
& +2 \operatorname{Re}\left[\tilde{g}_{h_{i} q \bar{q}}^{P}\left(\delta_{\text {sub }}^{P}\left(h_{i} \rightarrow q \bar{q}\right)\right)^{*}\right] .
\end{aligned}
$$

The strict one-loop factor $\delta \mathbf{Z}^{1 l}$ is given by

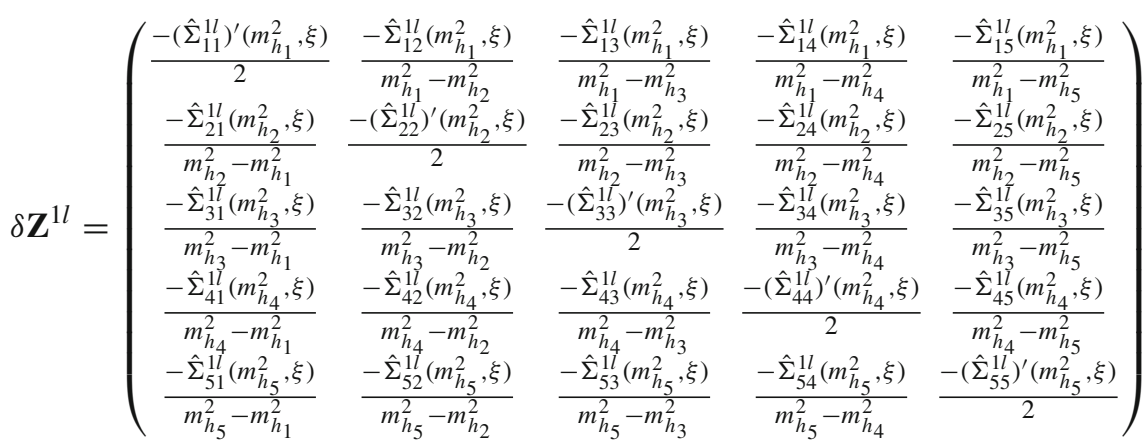

and

$$
\begin{aligned}
\Gamma_{H_{i} \rightarrow q \bar{q}}^{P, 1 l}= & \left(\tilde{g}_{h_{i} q \bar{q}}^{P}\right)\left(\tilde{g}_{h_{i} q \bar{q}}^{P}\right)^{*} \\
& +2 \operatorname{Re}\left[\tilde { g } _ { h _ { i } q \overline { q } } ^ { P } \left(\delta \mathcal{M}^{\mathrm{rem}, P}\left(h_{i} \rightarrow q \bar{q}\right)\right.\right.
\end{aligned}
$$

where $\hat{\Sigma}_{i j}^{1 l}\left(p^{2}\right)$ is the one-loop renormalized Higgs selfenergy of the transition $h_{i} \rightarrow h_{j}$ and $\left(\hat{\Sigma}^{1 l}\left(p^{2}\right)\right)^{\prime}=$ $\partial_{p^{2}} \hat{\Sigma}^{1 l}\left(p^{2}\right)$. The external Higgs mass is set to be the treelevel mass. Although the individual terms $\delta \mathcal{M}^{\mathrm{rem}, S / P}, \delta \mathbf{Z}_{i k}^{1 l}$, and $\delta \mathcal{M}_{G Z \text {,mix }}$ are gauge dependent, we have explicitely checked that the final result $\Gamma_{H_{i} \rightarrow q \bar{q}}^{S / P, 1 l}$ is indeed gaugeparameter independent.

These terms from the EW corrections are then combined with phase-space factors (and QCD corrections) to obtain 
Table 7 Mass values in $\mathrm{GeV}$ and main components of the neutral Higgs bosons at tree level, one-loop level, two-loop $\mathcal{O}\left(\alpha_{t} \alpha_{s}\right)$ level, and at two-loop $\mathcal{O}\left(\alpha_{t} \alpha_{s}+\alpha_{t}^{2}\right)$ level obtained by using OS renormalization in the top/stop sector

Table 8 Same as in Table 7 but using $\overline{\mathrm{DR}}$ renormalization in the top/stop sector

\begin{tabular}{llllll}
\hline & $h_{1} / H_{1}$ & $h_{2} / H_{2}$ & $h_{3} / H_{3}$ & $h_{4} / H_{4}$ & $h_{5} / H_{5}$ \\
\hline Tree-level & 58.63 & 87.34 & 157.40 & 704.25 & 708.97 \\
One-loop & 76.95 & 133.48 & 147.89 & 705.24 & 708.22 \\
Two-loop $\mathcal{O}\left(\alpha_{t} \alpha_{s}\right)$ & 77.03 & 117.69 & 147.74 & 704.83 & 708.34 \\
Two-loop $\mathcal{O}\left(\alpha_{t} \alpha_{s}+\alpha_{t}^{2}\right)$ & 77.11 & 124.83 & 147.74 & 705.03 & 708.34 \\
Main component & $h_{s}$ & $h_{u}$ & $a_{s}$ & $h_{d}$ & $a$
\end{tabular}

\begin{tabular}{llllll}
\hline & $h_{1} / H_{1}$ & $h_{2} / H_{2}$ & $h_{3} / H_{3}$ & $h_{4} / H_{4}$ & $h_{5} / H_{5}$ \\
\hline Tree-level & 58.63 & 87.34 & 157.40 & 704.25 & 708.97 \\
One-loop & 76.79 & 112.86 & 147.68 & 704.59 & 708.34 \\
Two-loop $\mathcal{O}\left(\alpha_{t} \alpha_{s}\right)$ & 76.8 & 118.25 & 147.74 & 704.7 & 708.29 \\
Two-loop $\mathcal{O}\left(\alpha_{t} \alpha_{s}+\alpha_{t}^{2}\right)$ & 76.8 & 118.34 & 147.74 & 704.7 & 708.29 \\
Main component & $h_{s}$ & $h_{u}$ & $a$ & $a_{s}$ & $h_{d}$ \\
\hline
\end{tabular}

the final decay widths. To illustrate the gauge dependence of these decay widths, we deliberately take a parameter point for which not only the $h_{u}$-like (SM-like) Higgs boson but also the singlet-like Higgs bosons are light. The soft-SUSY breaking parameters are given by

$$
\begin{aligned}
m_{\tilde{u}_{R}, \tilde{c}_{R}} & =m_{\tilde{d}_{R}, \tilde{s}_{R}, \tilde{b}_{R}}=m_{\tilde{Q}_{1,2}}=m_{\tilde{L}_{1,2,3}} \\
& =m_{\tilde{e}_{R}, \tilde{\mu}_{R}, \tilde{\tau}_{R}}=3 \mathrm{TeV} \\
m_{\tilde{t}_{R}} & =1576 \mathrm{GeV}, m_{\tilde{Q}_{3}}=1877 \mathrm{GeV}, \\
\left|A_{u, c, t}\right| & =2132 \mathrm{GeV},\left|A_{d, s, b}\right|=2000 \mathrm{GeV}, \\
\left|A_{e, \mu, \tau}\right| & =2000 \mathrm{GeV}, \\
\left|M_{1}\right| & =763 \mathrm{GeV},\left|M_{2}\right|=909 \mathrm{GeV},\left|M_{3}\right|=2231 \mathrm{GeV},
\end{aligned}
$$

with the CP phases given by

$$
\varphi_{A_{u, c, t}}=\varphi_{A_{d, s, b}}=\varphi_{A_{e, \mu, \tau}}=\varphi_{M_{1}}=\varphi_{M_{2}}=\varphi_{M_{3}}=0 .
$$

The remaining input parameters have been set to

$$
\begin{aligned}
\lambda & =0.478, \quad \kappa=-0.099, \quad \operatorname{Re}\left(A_{\kappa}\right)=207 \mathrm{GeV}, \\
\mu_{\mathrm{eff}} & =232.1 \mathrm{GeV}, \varphi_{u}=0, \quad \tan \beta=2.55, \\
M_{H^{ \pm}} & =701 \mathrm{GeV} .
\end{aligned}
$$

The Higgs boson masses and their main compositions in terms of singlet/doublet and scalar/pseudoscalar components at tree level, one-loop level, as well as two-loop $\mathcal{O}\left(\alpha_{t} \alpha_{s}\right)$ and two-loop $\mathcal{O}\left(\alpha_{t} \alpha_{s}+\alpha_{t}^{2}\right)$ levels are given in Table 7 for OS renormalization and in Table 8 for $\overline{\mathrm{DR}}$ renormalization in the top/stop sector. They have been computed with NMSSMCALCEW in the 'tHooft-Feynman gauge $(\xi=1)$. In this table, lower case $h_{i}$ refer to the tree-level mass eigenstates and capital $H_{i}$ refer to the loop-corrected mass eigenstates. As required by the choice of our scenario, the three light states are the two $\mathrm{CP}$-even and $\mathrm{CP}$-odd singlet-like
Higgs bosons and the $h_{u}$-like Higgs boson. Their masses receive large one-loop corrections, whereas the heavier Higgs bosons are practically insensitive to higher-order corrections to their masses.

In order to illustrate the gauge-parameter dependence of the loop-corrected Higgs masses (we have set $\xi_{W}=\xi_{Z}=$ $\xi$ ) we vary the parameter $\xi$ from 0 to 100 . We display in Fig. 15 (upper plot) the gauge-parameter dependence of the CP-even singlet-like Higgs-boson mass $M_{h_{s}}$ (red lines), of the SM-like Higgs-boson mass $M_{h_{u}}$ (blue lines), and of the CP-odd singlet-like Higgs-boson mass $M_{a_{s}}$ (black lines). The calculation is performed up to the two-loop order including the $\mathcal{O}\left(\alpha_{t} \alpha_{s}+\alpha_{t}^{2}\right)$ corrections. The full lines (dashed-lines) are obtained using the OS $(\overline{\mathrm{DR}})$ renormalization scheme in the top/stop sector. The lower plot displays the relative difference between the mass calculated in a general $R_{\xi}$ gauge, $M_{x}(\xi)$, and in the 'tHooft-Feynman gauge $\xi=1, M_{x}(\xi=1)$,

$$
\begin{aligned}
\Delta_{\xi}^{M} & =\frac{\left|M_{x}(\xi)-M_{x}(\xi=1)\right|}{M_{x}(\xi=1)}, \quad \text { with } \\
x & =h_{s}, h_{u}, a_{s}\left(\equiv H_{1}, H_{2}, H_{3}\right),
\end{aligned}
$$

as a function of $\xi$. It is clear from Fig. 15 that the $\xi$ dependence of singlet-like Higgs-boson masses is negligible, while the $h_{u}$-like Higgs boson mass has a significant gauge-parameter dependence, up to $9 \%$ at $\xi=100$.

We now investigate in detail the decay widths $\Gamma\left(H_{i} \rightarrow\right.$ $f \bar{f})$. As mentioned above, the pure one-loop corrections presented in Eqs. (5.27) and (5.28) are independent of $\xi$, where we set the external Higgs masses to their tree-level values. Hence, the only gauge dependence appearing in the decay widths calculated with Eq. (4.12) comes from the two-body phase-space factors evaluated with loop-corrected Higgs masses. We quantify the pure SUSY-EW correction 

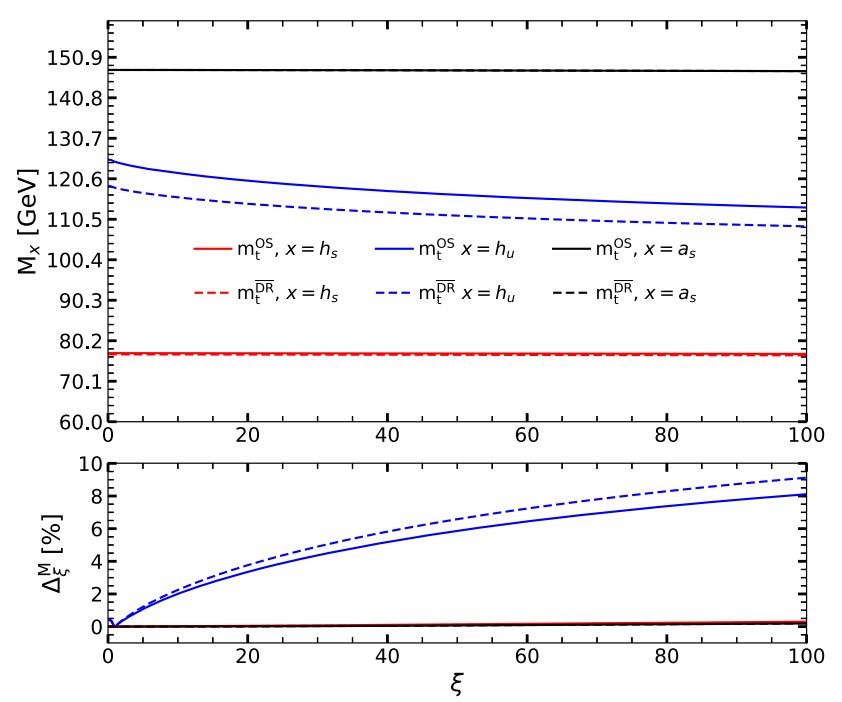

Fig. 15 Upper panel: The CP-even singlet-like (red), the SM-like (blue), and the $\mathrm{CP}$-odd singlet-like (black) masses as a function of the gauge parameter $\xi$ at two-loop $\mathcal{O}\left(\alpha_{t} \alpha_{s}+\alpha_{t}^{2}\right)$ level in the OS (solid lines) and the $\overline{\mathrm{DR}}$ (dashed lines) scheme of the top/stop sector. The dashed black line is hidden under the solid black line. Lower panel: Absolute value of the relative $\xi$ dependence of the loop-corrected masses $\Delta_{\xi}^{M}$ ( $x=h_{s}, h_{u}, a_{s}$ ) defined in the main text in Eq. (5.33), in percent, as a function of $\xi$. The color code in the lower plot is the same as in the upper plot

by

$\delta_{\text {pure }}^{\text {SEW }}=\frac{\left(\Gamma^{11}\left(H_{i} \rightarrow q \bar{q}\right)-\Gamma^{\text {tree }}\left(H_{i} \rightarrow q \bar{q}\right)\right)}{\Gamma^{\operatorname{tree}}\left(H_{i} \rightarrow q \bar{q}\right)}$

where the NLO decay width $\Gamma_{H_{i} \rightarrow q \bar{q}}^{11}$ is computed using Eq. (4.12) wherein the loop-corrected widths $\Gamma_{H_{i} \rightarrow q \bar{q}}^{S / P, 1 l}$ contain pure EW one-loop corrections as calculated with Eq. (5.27) and Eq. (5.28), respectively. The tree-level decay width is computed with the tree-level expressions

$\Gamma_{H_{i} \rightarrow q \bar{q}}^{\mathrm{S}, \text { tree }}=\left(\tilde{g}_{h_{i} q \bar{q}}^{S}\right)\left(\tilde{g}_{h_{i} q \bar{q}}^{S}\right)^{*}$

$\Gamma_{H_{i} \rightarrow q \bar{q}}^{\mathrm{P} \text { tree }}=\left(\tilde{g}_{h_{i} q \bar{q}}^{P}\right)\left(\tilde{g}_{h_{i} q \bar{q}}^{P}\right)^{*}$.

We also define the relative gauge dependence of the LO and NLO decay widths by

$\Delta_{\xi}^{\Gamma}=\frac{\Gamma_{\xi}-\Gamma_{\xi=1}}{\Gamma_{\xi=1}}$.

The results for the $\mathrm{CP}$-even singlet-like Higgs bosons are displayed in Fig. 16 (left plots), where the decay widths into $b \bar{b}$ (red lines), $c \bar{c}$ (blue lines), and $\tau^{+} \tau^{-}$(black lines) are presented (upper plot), as well as the corresponding pure SUSYEW corrections $\delta$ pure (middle plot) and the relative gauge dependence of the decay widths $\Delta_{\xi}^{\Gamma}$ (lower plot). The solid lines correspond to the LO results, the dashed lines to the NLO results. As expected by the analysis carried out above for the Higgs boson masses, $\Delta_{\xi} \Gamma_{\xi}$ is found to be at the per- mille level for the singlet-like Higgs bosons $H_{h_{s}}$ ( $c f$. Fig. 16 (lower left plot)) and $H_{a_{s}}$, while the SM-like Higgs boson $H_{h_{u}}$ displays a gauge dependence up to $\sim 10 \%$ for large $\xi$ in $\Gamma\left(H_{h_{u}} \rightarrow b \bar{b}, \tau^{+} \tau^{-}\right)$, and up to $\sim 8 \%$ in $\Gamma\left(H_{h_{u}} \rightarrow c \bar{c}\right)$, these numbers are not shown in figures included in this section. This reflects the $\sim 10 \%$ gauge dependence observed in the calculation of the loop-corrected mass $M_{H_{h_{u}}}$.

The pure SUSY-EW corrections $\delta_{\text {pure }}^{\text {SEW }}$, on the other hand, do not depend on $\xi$. The corrections are moderate for the SM-like and the CP-odd singlet-like Higgs bosons, between $+3 \%$ and $-11 \%$, while they are huge for the CP-even singletlike Higgs boson: $+293 \%$ for $\Gamma\left(H_{h_{s}} \rightarrow b \bar{b}, \tau^{+} \tau^{-}\right),-100 \%$ for $\Gamma\left(H_{h_{s}} \rightarrow c \bar{c}\right)$ ( $c f$. Fig. 16 (middle left plot)). These large corrections render the NLO predictions unreliable. However, they are mainly due to the term $\sum_{k=1}^{5} \delta \mathbf{Z}_{i k}^{1 l} \tilde{g}_{h_{k} q \bar{q}}^{S}$ in Eq. (5.27), where the contribution from the mixing between the $h_{s}$ and $h_{u}$ states is dominant. This term is universal and appears at all orders in perturbation theory. It can thus be resummed to all orders. This is precisely what is done with the $\mathbf{Z}^{H}$ matrix in Eqs. (4.13) and (4.14). This resummation re-introduces a mixing of different orders in perturbation theory on top of the use of the loop-corrected masses, enhancing thereby the dependence on the gauge parameter as presented in the right plots of Fig. 16, where we display the corresponding SUSY-EW corrections with the resummed $\mathbf{Z}$-factor, $\delta$ SEW, instead of the pure SUSY-EW corrections. The SUSY-EW corrections are now reasonably moderate, but at the expense of a gauge dependence that reaches up to $50 \%$ for $\xi=100$, for the sub-dominant decay channel $H_{h_{s}} \rightarrow c \bar{c}$. The gauge dependence is much more moderate for the decays into $b$ quark and $\tau$-lepton pairs. For these decay channels the SUSYEW corrections are rather small and the gauge dependence is below $30 \%$ for $\xi$ values between 0 and 100 .

The same effects are found for the SM-like Higgs boson and for the CP-odd singlet-like Higgs boson, as displayed in Fig. 17 where, in the upper panels, the LO and NLO improved decay widths into $b$-quark, $c$-quark, and $\tau$-lepon pairs, computed using the $\mathbf{Z}^{H}$ matrix and the loop-corrected masses, are displayed for the SM-like Higgs boson (left) and for the $\mathrm{CP}$-odd singlet-like Higgs boson (right) as a function of the gauge parameter $\xi$. The corresponding SUSY-EW corrections $\delta^{\text {SEW }}$ are displayed in the middle panels while the relative dependence on $\xi$ is displayed in the lower panels. While the pure SUSY-EW corrections $\delta_{\text {pure }}^{\text {SEW for the SM-like Higgs }}$ boson are almost constant with respect to a variation of $\xi$ and between $-2 \%$ and $3 \%$ for $\xi=100$, depending on the decay channel, the SUSY-EW corrections improved $\delta$ SEW depend on $\xi$ as seen in Fig. 17 (left) and can reach $+30 \%$ at $\xi=100$. The relative gauge dependence $\Delta_{\xi}^{\Gamma}$ can now reach $-30 \%$ at $\xi=100$. The gauge-dependence of the loopcorrected masses and due to the mixing of different orders of perturbation theory induced by the $\mathbf{Z}^{H}$ matrix add up. The same happens for the CP-odd singlet-like Higgs boson, 


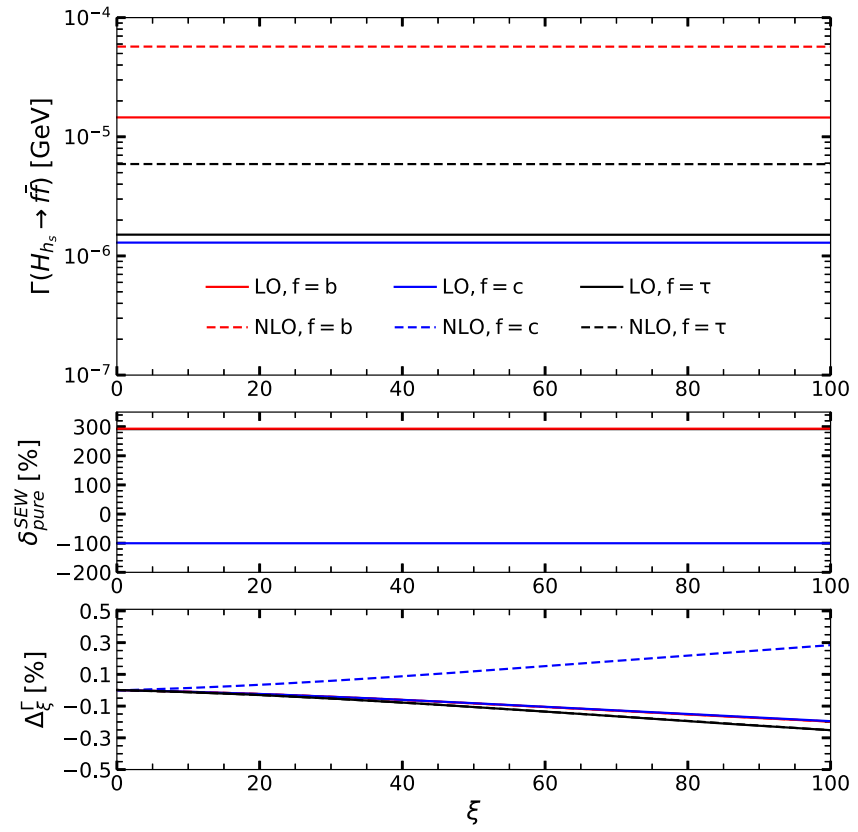

Fig. 16 Left plots: pure LO and NLO widths computed using Eqs. (5.27) and (5.28). Right plots: LO and NLO improved widths computed using the $\mathbf{Z}^{H}$ matrix and the loop-corrected masses as done in Eqs. (4.13) and (4.14). Top Panel: The CP-even singlet-like Higgs boson decay width into $b \bar{b}$ (red), $c \bar{c}$ (blue), $\tau \bar{\tau}$ (black) as a function of the gauge parameter $\xi$ at tree level (solid lines) and pure NLO level (dashed lines). The dashed blue line on the upper left plot is not shown since the width is negative and close to zero, about $-2.4 \times 10^{-9} \mathrm{GeV}$.
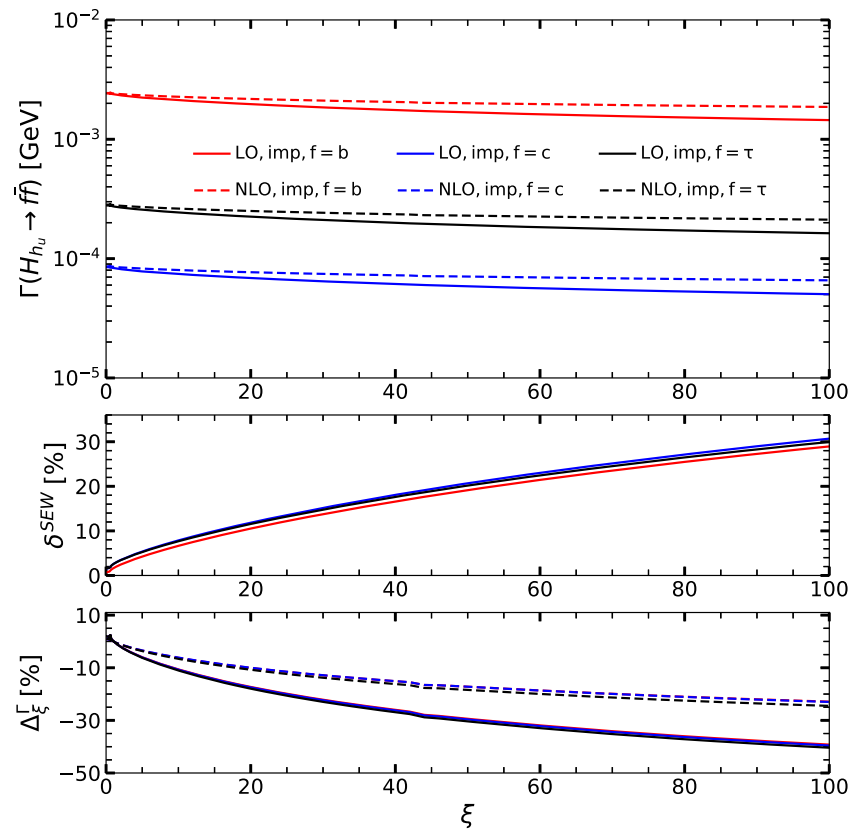

Fig. 17 Similar to the right-hand side of Fig. 16, but for the SM-like Higgs boson $H_{h_{u}}$ (left) and for the CP-odd singlet-like Higgs boson $H_{a_{s}}$ (right). In the lower left plot, the blue and red lines are hidden under the
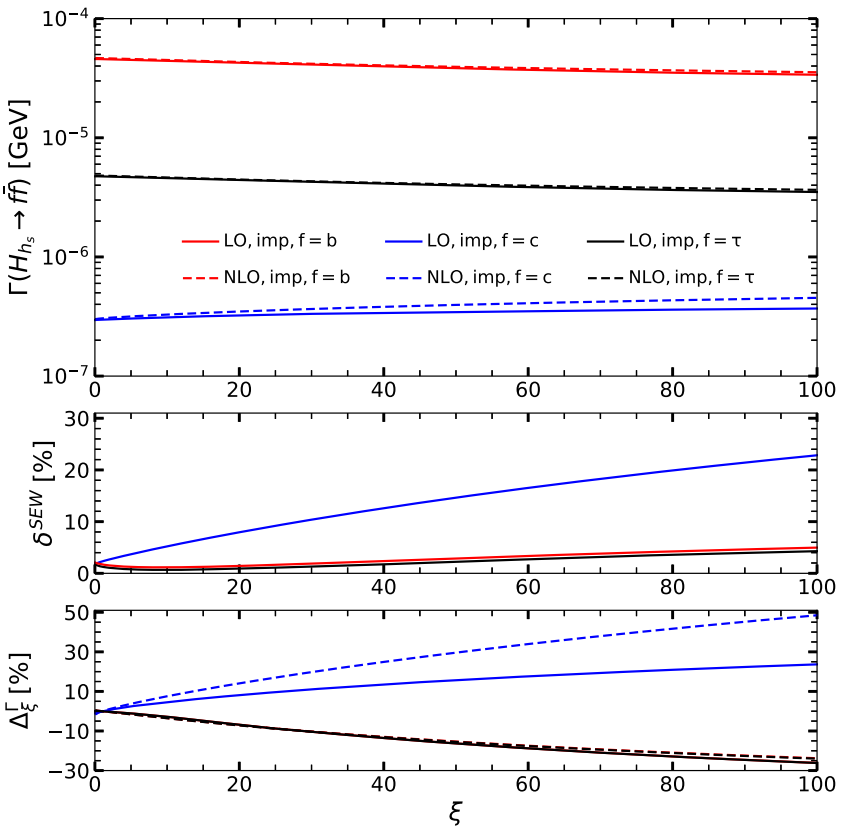

Middle Panel: Relative correction, defined as in Eq. (5.34), in percent, as a function of $\xi$. The black line is hidden under the red line in the middle left plot. Bottom Panel: Relative dependence of the widths on $\xi$, as defined in Eq. (5.36). In the lower left plot, the red solid and dashed lines are hidden under the blue solid line while the black dashed line is hidden under the black solid line. In the lower right plot, the red solid and dashed lines are hidden under the black solid and dashed lines, respectively
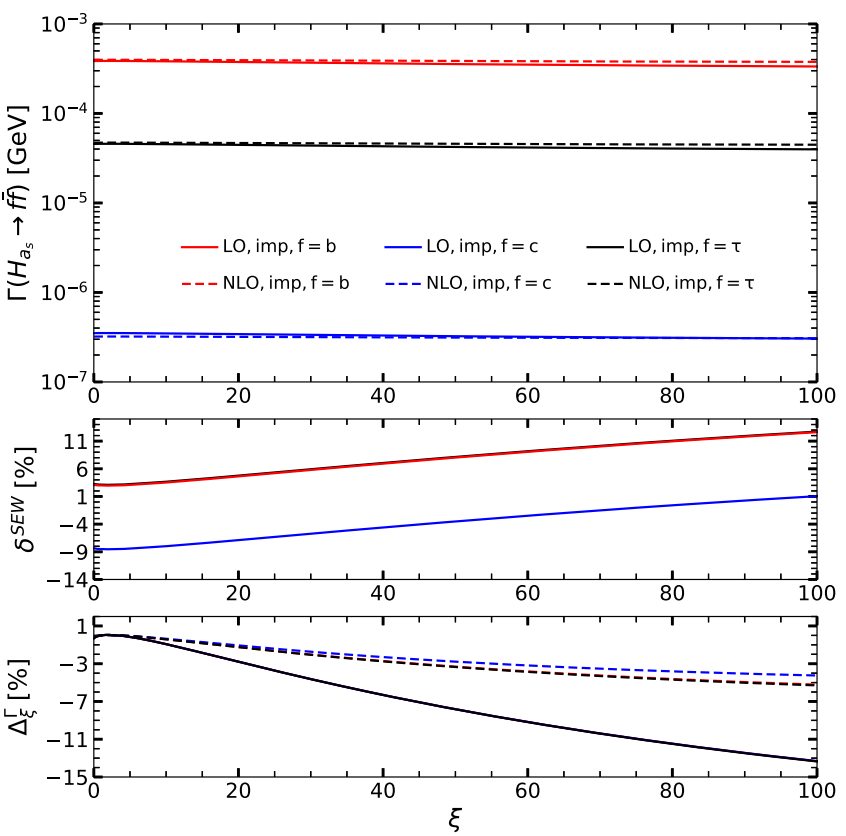

black solid line and the dashed red line is hidden under the dashed blue line. In the lower right plot, the red and blue solid lines are hidden under the black solid line, and the red dashed line under the black dashed line 
as seen on the right-hand side of Fig. 17, but with a more moderate gauge dependence as the gauge dependence of the loop-corrected mass is negligible. For the decays of the $H_{h_{u}}$ and $H_{a_{s}}$ Higgs bosons, we see a significant reduction of the relative gauge-parameter dependences of the NLO improved widths compared to the LO improved ones.

\section{Conclusions}

In this paper, we have presented in the framework of the CPviolating NMSSM our calculation of the complete one-loop (SUSY-)EW corrections to the on-shell Higgs boson decays into fermion pairs, massive gauge boson final states, gauge and Higgs boson final state pairs, electroweakino and stop and sbottom pairs. Where applicable we have included the SUSY-QCD corrections. We have implemented these new corrections into NMSSMCALC, a Fortran code for the computation of the Higgs mass spectrum up to presently two-loop order $\mathcal{O}\left(\alpha_{t} \alpha_{s}+\alpha_{t}^{2}\right)$ and the calculation of their branching ratios. The code already included in the branching ratios the state-of-the-art QCD corrections and the $\Delta_{b}$ corrections as well as decays into off-shell massive gauge boson pairs and decays with off-shell heavy quarks in the final state. The new code is called NMSSMCALCEW.

The consistent implementation of our newly computed corrections provides the presently highest level of precision in the calculation of the NMSSM Higgs boson decays. In contrast to the previous NMSSMCALC version, we have included the $\mathbf{Z}^{H}$ factor with full momentum dependence in order to render the loop-corrected Higgs fields on-shell. For the decays into the electroweakinos and into squark pairs different renormalization schemes were implemented. The numerical analysis has demonstrated that the relative change in the branching ratios due to this new treatment and the newly implemented corrections is significant. The analysis of the decays into chargino/neutralino and squark pairs for different renormalization schemes has shown that the oneloop corrections reduce the theoretical uncertainty due to missing higher-order corrections.

Finally, we have also discussed the issue of the gauge dependence in our calculation of the decay widths in the fermion-pair channels. We have explicitely shown that this originates from the loop-corrected masses used in the phasespace factor as well as from the $\mathbf{Z}^{H}$ factor, which both mix different orders in perturbation theory. Our nominal predictions for the loop-corrected Higgs masses and for the loop-corrected decay widths are calculated in the 'tHooftFeynman gauge. It should be noted that this gauge dependence only plays a significant role for light Higgs bosons.

The new code NMSSMCALCEW can be obtained at: https:// www.itp.kit.edu/ maggie/NMSSMCALCEW/.
Acknowledgements JB acknowledges the support from the Carl-Zeiss foundation. TND and MM acknowledge financial support from the DFG project Precision Calculations in the Higgs Sector - Paving the Way to the New Physics Landscape (ID: MU 3138/1-1). TND's work is funded by the Vietnam National Foundation for Science and Technology Development (NAFOSTED) under grant number 103.01-2017.78.

Data Availability Statement This manuscript has no associated data or the data will not be deposited. [Authors' comment: The work presented in this paper does not use any experimental data which needs to be deposited. All the numerical results are displayed in the figures and can be reproduced by running the computer code NMSSMCALCEW with the input parameters given in the paper.

Open Access This article is licensed under a Creative Commons Attribution 4.0 International License, which permits use, sharing, adaptation, distribution and reproduction in any medium or format, as long as you give appropriate credit to the original author(s) and the source, provide a link to the Creative Commons licence, and indicate if changes were made. The images or other third party material in this article are included in the article's Creative Commons licence, unless indicated otherwise in a credit line to the material. If material is not included in the article's Creative Commons licence and your intended use is not permitted by statutory regulation or exceeds the permitted use, you will need to obtain permission directly from the copyright holder. To view a copy of this licence, visit http://creativecomm ons.org/licenses/by/4.0/.

Funded by $\mathrm{SCOAP}^{3}$.

\section{A Counterterm contribution to the decay of a neutral Higgs boson into a squark pair}

In this appendix, we give explicit expressions of the counterterm couplings for the decays of a neutral Higgs boson into a squark pair. Note that we have used the redefined WFR constants for the squarks given in Eqs. (3.103) and (3.104). For the EW corrections, the counterterm entering Eq. (4.93) for the Higgs decay into a stop pair is given by

$$
\begin{aligned}
\mathcal{M}_{h_{i} \tilde{t}_{j} \tilde{t}_{k}^{*}}^{\mathrm{CT}, \mathrm{EW}}= & \frac{1}{2} \sum_{i^{\prime}=1}^{5} g_{h_{i^{\prime}} \tilde{t}_{j} \tilde{t}_{k}^{*}} \delta Z_{h_{i} h_{i^{\prime}}}+\frac{1}{2} \sum_{j^{\prime}=1}^{2} \\
& \times\left(g_{h_{i} \tilde{t}_{j^{\prime}} \tilde{t}_{k}^{*} \delta} \delta Z_{\tilde{t}_{j^{\prime}} \tilde{t}_{j}}\left(M_{\tilde{t}_{j}}^{2}\right)+g_{h_{i} \tilde{t}_{j} \tilde{t}_{j^{\prime}}^{\delta}} \delta Z_{\tilde{t}_{j^{\prime}} \tilde{t}_{k}}^{*}\left(M_{\tilde{t}_{k}}^{2}\right)\right) \\
& +g_{h_{i} \tilde{t}_{j} \tilde{t}_{k}^{*}}\left(\delta Z_{e}+\frac{\delta M_{Z}^{2}}{2 M_{Z}^{2}}-\frac{\delta s_{\theta_{W}}}{s_{\theta_{W}}}-\frac{\delta c_{\theta_{W}}}{c_{\theta_{W}}}\right) \\
& +\frac{e M_{Z}}{s_{\theta_{W}} c_{\theta_{W}}}\left[\frac{m_{t}^{2} \mathcal{R}_{i 2}\left(U_{j 1}^{\tilde{t} *} U_{k 1}^{\tilde{t}}+U_{j 2}^{\tilde{t} *} U_{k 2}^{\tilde{t}}\right)}{s_{\beta} M_{Z}^{2}}\right. \\
& \times\left(2 \frac{\delta m_{t}}{m_{t}}-\frac{\delta M_{Z}^{2}}{M_{Z}^{2}}-\frac{\delta s_{\beta}}{s_{\beta}}\right) \\
& +\frac{m_{t}\left(U_{j 2}^{\tilde{t} *} U_{k 1}^{\tilde{t}} F_{1}+U_{j 1}^{\tilde{t} *} U_{k 2}^{\tilde{t}} F_{1}^{*}\right)}{2 s_{\beta} M_{Z}^{2}}
\end{aligned}
$$




$$
\begin{aligned}
& \times\left(\frac{\delta m_{t}}{m_{t}}-\frac{\delta M_{Z}^{2}}{M_{Z}^{2}}-\frac{\delta s_{\beta}}{s_{\beta}}\right) \\
& +\frac{m_{t}\left(U_{j 2}^{\tilde{t} *} U_{k 1}^{\tilde{t}} \delta F_{1}+U_{j 1}^{\tilde{t} *} U_{k 2}^{\tilde{t}} \delta F_{1}^{*}\right)}{2 s_{\beta} M_{Z}^{2}} \\
& +\frac{1}{6}\left(\delta c_{\beta} \mathcal{R}_{i 1}-\delta s_{\beta} \mathcal{R}_{i 2}\right) \\
& \times\left(\left(4 c_{\theta_{W}}^{2}-1\right) U_{j 1}^{\tilde{t} *} U_{k 1}^{\tilde{t}}+4 s_{\theta_{W}}^{2} U_{j 2}^{\tilde{t} *} U_{k 2}^{\tilde{t}}\right) \\
& +\frac{4}{3}\left(c_{\beta} \mathcal{R}_{i 1}-s_{\beta} \mathcal{R}_{i 2}\right) \\
& \left.\times\left(-U_{j 1}^{\tilde{t} *} U_{k 1}^{\tilde{t}}+U_{j 2}^{\tilde{t} *} U_{k 2}^{\tilde{t}}\right) s_{\theta_{W}} \delta s_{\theta_{W}}\right],
\end{aligned}
$$

and for the decay into a sbottom pair

$$
\begin{aligned}
& \mathcal{M}_{h_{i} \tilde{b}_{j} \tilde{b}_{k}^{*}}^{\mathrm{CT}, \mathrm{WW}}= \frac{1}{2} \sum_{i^{\prime}=1}^{5} g_{h_{i^{\prime}} \tilde{b}_{j} \tilde{b}_{k}^{*}} \delta Z_{h_{i} h_{i^{\prime}}}+\frac{1}{2} \sum_{j^{\prime}=1}^{2} \\
& \times\left(g_{h_{i} \tilde{b}_{j^{\prime}} \tilde{b}_{k}^{*}} \delta Z_{\tilde{b}_{j^{\prime}} \tilde{b}_{j}}\left(M_{\tilde{b}_{j}}^{2}\right)+g_{h_{i} \tilde{b}_{j} \tilde{b}_{j^{\prime}}^{*}} \delta Z_{\tilde{b}_{j^{\prime}} \tilde{b}_{k}}^{*}\left(M_{\tilde{b}_{k}}^{2}\right)\right) \\
&+g_{h_{i} \tilde{b}_{j} \tilde{b}_{k}^{*}}\left(\delta Z_{e}+\frac{\delta M_{Z}^{2}}{2 M_{Z}^{2}}-\frac{\delta s_{\theta_{W}}}{s_{\theta_{W}}}-\frac{\delta c_{\theta_{W}}}{c_{\theta_{W}}}\right) \\
&+\frac{e M_{Z}}{s_{\theta_{W}} c_{\theta_{W}}}\left[\frac{m_{b}^{2} \mathcal{R}_{i 1}\left(U_{j 1}^{\tilde{b} *} U_{k 1}^{\tilde{b}}+U_{j 2}^{\tilde{b} *} U_{k 2}^{\tilde{b}}\right)}{c_{\beta} M_{Z}^{2}}\right. \\
& \times\left(2 \frac{\delta m_{b}}{m_{b}}-\frac{\delta M_{Z}^{2}}{M_{Z}^{2}}-\frac{\delta c_{\beta}}{c_{\beta}}\right) \\
&+\frac{m_{b}\left(U_{j 2}^{\tilde{b} *} U_{k 1}^{\tilde{b}} F_{2}+U_{j 1}^{\tilde{b} *} U_{k 2}^{\tilde{b}} F_{2}^{*}\right)}{2 c_{\beta} M_{Z}^{2}} \\
& \times\left(\frac{\delta m_{b}}{m_{b}}-\frac{\delta M_{Z}^{2}}{M_{Z}^{2}}-\frac{\delta c_{\beta}}{c_{\beta}}\right) \\
& \times-\frac{2}{3}\left(c_{\beta} \mathcal{R}_{i 1}-s_{\beta} \mathcal{R}_{i 2}\right) \\
& \times \frac{m_{b}\left(U_{j 2}^{\tilde{b} *} U_{k 1}^{\tilde{b}} \delta F_{2}+U_{j 1}^{\tilde{b} *} U_{k 2}^{\tilde{b}} \delta F_{2}^{*}\right)}{2 c_{\beta} M_{Z}^{2}} \\
&\left.\times \frac{1}{6}\left(\delta c_{\beta}^{\tilde{b} *} U_{k 1}^{\tilde{b}}+U_{j 2}^{\tilde{b} *} U_{k 2}^{\tilde{b}}\right) s_{\theta_{W}} \delta s_{\theta_{W}}-\delta s_{\beta} \mathcal{R}_{i 2}\right) \\
&\left.\left.\theta_{\theta_{W}}^{2}+1\right) U_{j 1}^{\tilde{b} *} U_{k 1}^{\tilde{b}}+2 s_{\theta_{W}}^{2} U_{j 2}^{\tilde{b} *} U_{k 2}^{\tilde{b}}\right) \\
& \\
& \\
&
\end{aligned}
$$

where

$$
\begin{aligned}
\delta F_{1}= & \delta A_{t}^{*} e^{-i \varphi_{u}}\left(\mathcal{R}_{i 2}-i c_{\beta} \mathcal{R}_{i 4}\right)-\delta \mu_{\mathrm{eff}}\left(\mathcal{R}_{i 1}+i s_{\beta} \mathcal{R}_{i 4}\right) \\
& -\frac{v \lambda c_{\beta}\left(\mathcal{R}_{i 3}+i \mathcal{R}_{i 5}\right) e^{i \varphi_{s}}}{\sqrt{2}}\left(\frac{\delta \lambda}{\lambda}+\frac{\delta v}{v}+\frac{\delta c_{\beta}}{c_{\beta}}\right)
\end{aligned}
$$

$$
\begin{aligned}
\delta F_{2}= & \delta A_{b}^{*}\left(\mathcal{R}_{i 1}-i s_{\beta} \mathcal{R}_{i 4}\right)-\delta \mu_{\mathrm{eff}} e^{i \varphi_{u}}\left(\mathcal{R}_{i 2}+i c_{\beta} \mathcal{R}_{i 4}\right) \\
& -\frac{v \lambda s_{\beta}\left(\mathcal{R}_{i 3}+i \mathcal{R}_{i 5}\right) e^{i\left(\varphi_{s}+\varphi_{u}\right)}}{\sqrt{2}}\left(\frac{\delta \lambda}{\lambda}+\frac{\delta v}{v}+\frac{\delta s_{\beta}}{s_{\beta}}\right) .
\end{aligned}
$$

For the QCD corrections, the counterterm couplings are obtained from $\mathcal{M}_{h_{i} \tilde{q}_{j} \tilde{q}_{k}^{*}}^{\mathrm{CT}, \mathrm{EW}}$ by setting the set of EW counterterms, $\delta Z_{e}, \delta M_{Z}^{2}, \delta v, \delta v_{s}, \delta \lambda, \delta s_{\theta_{W}}, \delta c_{\theta_{W}}, \delta s_{\beta}, \delta c_{\beta}$ and $\delta Z_{h_{i} h_{i^{\prime}}}$ to zero. Note that the counterterm $\delta \mu_{\text {eff }}$ is given in terms of $\delta \lambda$ and $\delta v_{s}$ as

$\delta \mu_{\mathrm{eff}}=e^{i \varphi_{s}} \frac{\left(v_{s} \delta \lambda+\lambda \delta v_{s}\right)}{\sqrt{2}}$.

\section{B The code NMSSMCALCEW}

We here give a brief introduction into our new code NMSSMCALCEW that includes the newly calculated and here presented corrections to the decay widths of the neutral NMSSM Higgs bosons in the CP-violating NMSSM, as well as the newly calculated and here presented one-loop corrections to the chargino, neutralino, stop, and sbottom masses. It is derived from the code NMSSMCALC, which is described in detail in Ref. [14]. We here concentrate on the new features in NMSSMCALCEW with respect to NMSSMCALC.

NMSSMCALCEW requires 'LoopTools' version 2.14 (or higher) $[42,88]$ to work with the EW corrections in the decay part. If 'LoopTools' is not installed yet, it can be obtained from the url: http://www.feynarts.de/looptools/

In order to generate the executable, download and unpack the tar file 'nmssmcalcew.tar.gz'. It contains two subdirectories called 'nmssmcalc_rew_alphat2-master' and 'nmssmcalc_cew_alphat2-master' for the real and complex NMSSM, respectively. Go to the subdirectory of the version in which you want to work in. Open in a text editor the file 'makefile' and in line 31 provide the absolute path to the 'LoopTools' binary directory located in the main directory of LoopTools. Modify also the line 66 (to make sure it refers to the correct 'lib' sub-directory within the 'LoopTools' binary directory). In case the package is compiled without the EW corrections in the decay widths, the flag 'yesEW' can be switched to 'FALSE' on line 19 and 'LoopTools' is not needed anymore. Subsequently, all files are compiled by typing 'make'. An executable 'run' is created. By typing'./run' the executable is run.

For the code to be run, the user has to provide the input files for 'CalcMasses.F' (default name 'inp.dat') and for bhdecay(_c).f (to be named 'bhdecay.in'). The user also has the choice to provide in the command line the names of the input and output files for 'CalcMasses.F' (first and second argument) and the name of the output file provided by the decay routine (third argument). Hence the command will be 'run 
name_file1 name_file2 name_file 3' in this case. Sample input files 'inp.dat' and 'bhdecay.in' are included in the .tar files. By typing 'make clean' the executable as well as the object files generated in the 'obj' directory are removed.

In 'bhdecay.in' that is used by NMSSMCALCEW, new options have been included. They are

- 'ischhXX' to choose the renormalization scheme for the loop corrected electroweakino masses. The options are 1 (OS1), 2 (OS2) and 3 (DRbar). The two OS schemes are specified in Sect. 3.1.2.

- 'ischhst' to choose the renormalization scheme for the stop sector. The options are 1 (OS NLO-EW, OS NLOQCD), 2 (OS NLO-EW, DRbar NLO-QCD), 3 (DRbar NLO-EW, DRbar NLO-QCD).

- 'ischhsb' to choose the renormalization scheme for the sbottom sector. The options are 1 (OS NLO-EW, OS NLO-QCD), 2 (OS NLO-EW, DRbar NLO-QCD), 3 (DRbar NLO-EW, DRbar NLO-QCD).

- 'iewh' to choose the levels of NLO SUSY-EW (SUSYQCD) corrections that are included. The options are 0 (as in NMSSMCALC 3.00), 1 (decays as implemented in NMSSMCALC, but with the $\mathbf{Z}^{H}$ factor), 2 (full NLO corrections as described in this paper). Both for option 1 and 2, the loop-corrected electroweakino and stop/sbottom masses are used in the phase space factor.

Further information on the organization of the files for the code and their functionalities as well as modifications on the code (which are constantly updated) can be found at the webpage of NMSSMCALCEW. The code has been tested on a Linux machine.

\section{References}

1. ATLAS, G. Aad et al., Phys. Lett. B 716, 1 (2012). arXiv:1207.7214

2. CMS, S. Chatrchyan et al., Phys. Lett. B 716, 30 (2012). arXiv: 1207.7235

3. ATLAS, CMS, G. Aad et al., Phys. Rev. Lett. 114, 191803 (2015). arXiv: 1503.07589

4. M. Maniatis, Int. J. Mod. Phys. A 25, 3505 (2010). arXiv:0906.0777

5. U. Ellwanger, C. Hugonie, A.M. Teixeira, Phys. Rept. 496, 1 (2010). arXiv:0910.1785

6. J. Baglio, C.O. Krauss, M. Mühlleitner, K. Walz, JHEP 1510, 024 (2015). arXiv: 1505.07125

7. G. Bélanger, V. Bizouard, F. Boudjema, G. Chalons, Phys. Rev. D 93, 115031 (2016). arXiv:1602.05495

8. G. Bélanger, V. Bizouard, F. Boudjema, G. Chalons, Phys. Rev. D 96, 015040 (2017). arXiv:1705.02209

9. M.D. Goodsell, S. Liebler, F. Staub, Eur. Phys. J. C 77, 758 (2017). arXiv: 1703.09237

10. F. Domingo, S. Heinemeyer, S. Paßehr, G. Weiglein, Eur. Phys. J. C 78, 942 (2018). arXiv: 1807.06322

11. D.T. Nhung, M. Mühlleitner, J. Streicher, K. Walz, JHEP 1311, 181 (2013). arXiv:1306.3926
12. M. Mühlleitner, D.T. Nhung, H. Ziesche, JHEP 1512, 034 (2015). arXiv: 1506.03321

13. F. Domingo, S. Paßehr, Eur. Phys. J. C 79, 905 (2019). arXiv: 1907.05468

14. J. Baglio et al., Comput. Phys. Commun. 185, 3372 (2014). arXiv: 1312.4788

15. K. Ender, T. Graf, M. Mühlleitner, H. Rzehak, Phys. Rev. D 85, 075024 (2012). arXiv: 1111.4952

16. T. Graf, R. Gröber, M. Mühlleitner, H. Rzehak, K. Walz, JHEP 1210, 122 (2012). arXiv: 1206.6806

17. M. Mühlleitner, D.T. Nhung, H. Rzehak, K. Walz, JHEP 1505, 128 (2015). arXiv:1412.0918

18. T.N. Dao, R. Gröber, M. Krause, M. Mühlleitner, H. Rzehak, JHEP 1908, 114 (2019). arXiv:1903.11358

19. M. Kobayashi, T. Maskawa, Prog. Theor. Phys. 49, 652 (1973)

20. W. Siegel, Phys. Lett. B 84, 193 (1979)

21. D. Stockinger, JHEP 0503, 076 (2005). arXiv:hep-ph/0503129

22. A. Bharucha, A. Fowler, G. Moortgat-Pick, G. Weiglein, JHEP 1305, 053 (2013). arXiv:1211.3134

23. D. Espriu, J. Manzano, P. Talavera, Phys. Rev. D 66, 076002 (2002). arXiv:hep-ph/0204085

24. A.C. Fowler, G. Weiglein, JHEP 1001, 108 (2010). arXiv:0909.5165

25. A. Denner, Fortsch. Phys. 41, 307 (1993). arXiv:0709.1075

26. T. Fritzsche, W. Hollik, Eur. Phys. J. C 24, 619 (2002). arXiv:hep-ph/0203159

27. J. Guasch, W. Hollik, J. Sola, JHEP 0210, 040 (2002). arXiv:hep-ph/0207364

28. N. Baro, F. Boudjema, Phys. Rev. D 80, 076010 (2009). arXiv:0906.1665

29. A. Chatterjee, M. Drees, S. Kulkarni, Q. Xu, Phys. Rev. D 85, 075013 (2012). arXiv: 1107.5218

30. K. Williams, G. Weiglein, Phys. Lett. B 660, 217 (2008). arXiv:0710.5320

31. D. Pierce, A. Papadopoulos, Phys. Rev. D 50, 565 (1994). arXiv:hep-ph/9312248

32. H. Eberl, M. Kincel, W. Majerotto, Y. Yamada, Phys. Rev. D 64, 115013 (2001). arXiv:hep-ph/0104109

33. S.P. Martin, Phys. Rev. D 72, 096008 (2005). arXiv:hep-ph/0509115

34. J. Küblbeck, M. Böhm, A. Denner, Comput. Phys. Commun. 60, 165 (1990)

35. T. Hahn, Comput. Phys. Commun. 140, 418 (2001). arXiv:hep-ph/0012260

36. F. Staub, Comput. Phys. Commun. 181, 1077 (2010). arXiv:0909.2863

37. F. Staub, Comput. Phys. Commun. 182, 808 (2011). arXiv: 1002.0840

38. F. Staub, Comput. Phys. Commun. 184, 1792 (2013). arXiv: 1207.0906

39. F. Staub, Comput. Phys. Commun. 185, 1773 (2014). arXiv: 1309.7223

40. R. Mertig, M. Böhm, A. Denner, Comput. Phys. Commun. 64, 345 (1991)

41. V. Shtabovenko, R. Mertig, F. Orellana, Comput. Phys. Commun. 207, 432 (2016). arXiv:1601.01167

42. T. Hahn, M. Perez-Victoria, Comput. Phys. Commun. 118, 153 (1999). arXiv:hep-ph/9807565

43. M. Carena, D. Garcia, U. Nierste, C.E.M. Wagner, Nucl. Phys. B 577, 88 (2000). arXiv:hep-ph/9912516

44. M. Carena, J.R. Ellis, S. Mrenna, A. Pilaftsis, C.E.M. Wagner, Nucl. Phys. B 659, 145 (2003). arXiv:hep-ph/0211467

45. J. Guasch, P. Hafliger, M. Spira, Phys. Rev. D 68, 115001 (2003). arXiv:hep-ph/0305101

46. R. Hempfling, Phys. Rev. D 49, 6168 (1994) 
47. L.J. Hall, R. Rattazzi, U. Sarid, Phys. Rev. D 50, 7048 (1994). arXiv:hep-ph/9306309

48. M. Carena, M. Olechowski, S. Pokorski, C. Wagner, Nucl. Phys. B 426, 269 (1994). arXiv:hep-ph/9402253

49. D.M. Pierce, J.A. Bagger, K.T. Matchev, R. Zhang, Nucl. Phys. B 491, 3 (1997). arXiv:hep-ph/9606211

50. M. Carena, S. Mrenna, C.E.M. Wagner, Phys. Rev. D 60, 075010 (1999). arXiv:hep-ph/9808312

51. D. Noth, M. Spira, Phys. Rev. Lett. 101, 181801 (2008). arXiv:0808.0087

52. L. Mihaila, C. Reisser, JHEP 1008, 021 (2010). arXiv:1007.0693

53. E. Braaten, J.P. Leveille, Phys. Rev. D 22, 715 (1980)

54. N. Sakai, Phys. Rev. D 22, 2220 (1980)

55. T. Inami, T. Kubota, Nucl. Phys. B 179, 171 (1981)

56. M. Drees, Ki Hikasa, Phys. Lett. B 240, 455 (1990). [Erratum: Phys. Lett .B 262, 497 (1991)]

57. DYu. Bardin, P.K. Khristova, B.M. Vilensky, Sov. J. Nucl. Phys. 54, 833 (1991) [Yad. Fiz. 54, 1366 (1991)]

58. A. Dabelstein, W. Hollik, Z. Phys. C 53, 507 (1992)

59. A. Dabelstein, Nucl. Phys. B 456, 25 (1995). arXiv:hep-ph/9503443

60. A. Djouadi, J. Kalinowski, M. Spira, Comput. Phys. Commun. 108, 56 (1998). arXiv:hep-ph/9704448

61. A. Djouadi, J. Kalinowski, M. Muehlleitner, M. Spira, Comput. Phys. Commun. 238, 214 (2019). arXiv:1801.09506

62. M. Drees, K.-I. Hikasa, Phys. Rev. D 41, 1547 (1990)

63. S.G. Gorishnii, A.L. Kataev, S.A. Larin, L.R. Surguladze, Mod. Phys. Lett. A 5, 2703 (1990)

64. S.G. Gorishnii, A.L. Kataev, S.A. Larin, L.R. Surguladze, Phys. Rev. D 43, 1633 (1991)

65. A.L. Kataev, V.T. Kim, Mod. Phys. Lett. A 9, 1309 (1994)

66. S.G. Gorishnii, A.L. Kataev, S.A. Larin, Sov. J. Nucl. Phys. 40, 329 (1984) [Yad. Fiz. 40, 517 (1984)]

67. L.R. Surguladze, Phys. Lett. B 341, 60 (1994). arXiv:hep-ph/9405325

68. S.A. Larin, T. van Ritbergen, J.A.M. Vermaseren, Phys. Lett. B 362, 134 (1995). arXiv:hep-ph/9506465
69. K.G. Chetyrkin, A. Kwiatkowski, Nucl. Phys. B 461, 3 (1996). arXiv:hep-ph/9505358

70. K.G. Chetyrkin, Phys. Lett. B 390, 309 (1997). arXiv:hep-ph/9608318

71. P.A. Baikov, K.G. Chetyrkin, J.H. Kuhn, Phys. Rev. Lett. 96, 012003 (2006). arXiv:hep-ph/0511063

72. K.E. Williams, H. Rzehak, G. Weiglein, Eur. Phys. J. C 71, 1669 (2011). arXiv:1103.1335

73. B.A. Kniehl, Nucl. Phys. B 357, 439 (1991)

74. T.N. Dao, L. Fritz, M. Krause, M. Mühlleitner, S. Patel, Eur. Phys. J. C 80, 292 (2020). arXiv:1911.07197

75. P. Gonzalez, S. Palmer, M. Wiebusch, K. Williams, Eur. Phys. J. C 73, 2367 (2013). arXiv:1211.3079

76. W. Hollik, J.-H. Zhang, Phys. Rev. D 84, 055022 (2011). arXiv: 1109.4781

77. F. Domingo, S. Paßehr, (2020). arXiv:2007.11010 [hep-ph]

78. P. Bechtle, O. Brein, S. Heinemeyer, G. Weiglein, K.E. Williams, Comput. Phys. Commun. 181, 138 (2010). arXiv:0811.4169

79. P. Bechtle, O. Brein, S. Heinemeyer, G. Weiglein, K.E. Williams, Comput. Phys. Commun. 182, 2605 (2011). arXiv:1102.1898

80. P. Bechtle et al., Eur. Phys. J. C 74, 2693 (2014). arXiv:1311.0055

81. P. Bechtle, S. Heinemeyer, O. Stål, T. Stefaniak, G. Weiglein, Eur. Phys. J. C 74, 2711 (2014). arXiv:1305.1933

82. J. Baglio et al., EPJ Web Conf. 49, 12001 (2013)

83. Particle Data Group, M. Tanabashi et al., Phys. Rev. D 98, 030001 (2018)

84. A. Denner et al., LHCHXSWG-INT-2015-006 (2015)

85. P.Z. Skands et al., JHEP 0407, 036 (2004). arXiv:hep-ph/0311123

86. CMS, V. Khachatryan et al., Phys. Lett. B 755, 217 (2016). arXiv: 1510.01181

87. CMS, V. Khachatryan et al., Phys. Lett. B 759, 369 (2016). arXiv: 1603.02991

88. G.J. van Oldenborgh, J.A.M. Vermaseren, Z. Phys. C 46, 425 (1990) 